\title{
Soporte al diagnóstico de TDAH por búsqueda automatizada de patrones en el EEG
}

\author{
Tesis que para obtener el grado de \\ Maestro en Ciencias (Ingeniería Biomédica) \\ Presenta \\ Ing. Biom. Federico Alba-Sánchez.
}

Julio 8, 2011

Asesor: M. en I. Oscar Yañez Suárez

Sinodales:

Dr. Héctor Brust Carmona

Instituto Nacional de Rahabilitación

M. en I. Fabiola Margarita Martínez Licona Depto. de Ingeniería Eléctrica, UAM-I

M. en I. Oscar Yañez Suárez

Depto. de Ingeniería Eléctrica, UAM-I
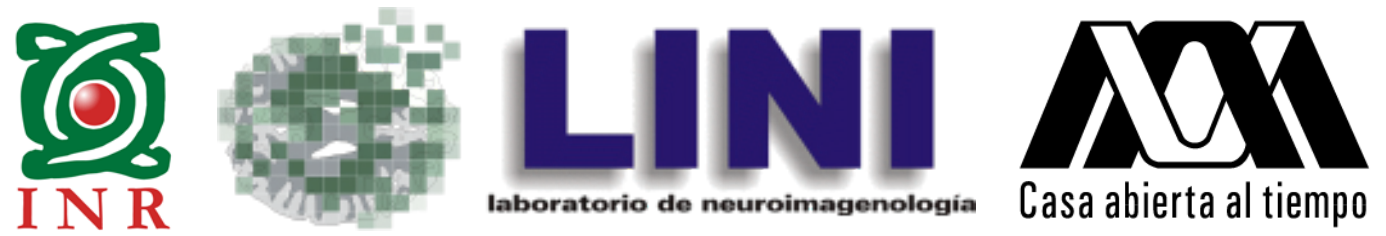

Universidad Autónoma Metropolitana

Unidad Iztapalapa

Ciudad de México 
En el fondo, los cientificos somos gente con suerte: podemos jugar a lo que queramos durante toda la vida.

LEE SMOLIN

\section{Resumen}

El electroencefalograma continúa siendo una herramienta atractiva en la práctica clínica debido a que proporciona medidas bioeléctricas objetivas de la actividad contínua del cerebro, a su no invasividad y a su descripción en tiempo real el cual ha sido utilizado con éxito en el diagnóstico de desórdenes como la epilepsia y la esquizofrenia, entre algunas otras pudiendo también jugar un papel importante en el diagnóstico del Trastorno por Déficit de Atención con Hiperactividad.

Esta investigación se enfocó en el diseño y desarrollo de una herramienta automatizada de soporte para el diagnóstico del trastorno por déficit de atención con hiperactividad a partir únicamente de un conjunto de señales electroencefalográficas adquiridas bajo un protocolo específico de estimulación visual con lámpara flash y utilizando como base, el sistema internacional de colocación de electrodos 10/20.

La extracción de características fue sugerida por dos vías distintas la primera, mediante la determinación de índices de potencia espectrales dependientes de la ubicación espacial de la foto-estimulación aplicada durante la adquisición de las señales y la segunda, con un análisis multiresolución por medio de una descomposición completa en paquetes Wavelet estudiando en este caso, la energía asociada a cada conjunto de coeficientes.

Finalmente, el análisis de los rasgos extraídos se llevó a cabo mediante una arquitectura de clasificación compuesta primero, por dos capas de 10x10 nodos de Mapas auto-organizados segundo, por un posterior análisis de conglomerados a través del algoritmo de las $k$ medias y por último, definiendo regiones de probabilidad de presencia de la enfermedad sobre el mapa clasificador segmentado, asignando para ello una codificación en color para cada una de estas regiones.

La población de estudio analizada constó de 28 niños referidos para evaluación psicológica por parte de su escuela con edades entre los siete y doce años de los cuales, 18 fueron diagnosticados con TDAH (15 hombres y tres mujeres) y diez sin TDAH (cinco hombres y cinco mujeres) por la sección de Neuropsicología del Instituto Nacional de Rehabilitación en la ciudad de México basados en las pruebas estandarizadas DSMIV.

El mapa clasificador generado en esta investigación logró detectar sujetos con TDAH con una taza de acierto del $0,7 \pm 0,11$, a partir del conjunto de rasgos frecuenciales, y del 0,77 $\pm 0,15$, a partir de los rasgos wavelet, ambos para datos no vistos después de la aplicación de un proceso de validación cruzada a seis vías, esto nos prueba que ésta arquitectura de análisis cuantitativa podría apoyar al experto humano clínico durante su tarea diagnóstica. 
Por qué las cosas son como son y no de otra manera?

JOHANNES KEPLER

\section{Abstract}

The electroencephalogram is an attractive clinical tool given its non-invasive nature, its ability to reflect real-time changes in local cortical activity, and the load of objective bioelectrical measurements that can be derived from it.

For decades, the electroencephalogram has been successfully used for diagnosing epilepsy and schizophrenia, among other brain disorders. This research focuses in the design and implementation of a computer-aided diagnostic tool for establishing the likelihood of presence of Attention-Deficit Hyperactivity Disorder in children, out of routine electroencephalographic recordings obtained during a specific visual stimulation protocol.

Classical bandpower features and wavelet techniques from multiple differential recordings are computed and used as features in a classifier built from a cooperative ensemble of labeled selforganizing maps.

Studied population consisted of 28 children with ages between 7-12 years, eighteen of which were diagnosed with ADHD (15 male, three female) and ten without ADHD (five male, five female).

Subjects were referred by their educational institutions for psychological evaluation and ADHD diagnosis to the Neuropsychology section of the National Rehabilitation Institute in Mexico City. Evaluation was performed through the application of standardized DSM-IV questionaries and a clinical interview.

Classification accuracy of the proposed system is $0,7 \pm 0,11$ for bandpower features and $0,77 \pm 0,15$ for wavelet features, as estimated from unseen data, a result that points to the idea that such a quantitative diagnostic aid could adequately support the diagnostic task of a clinical expert. 
Haz lo necesario para lograr tu más ardiente deseo

y al final acabarás lográndolo. LUDWING VAN BEETHOVEN

\section{Agradecimientos}

La realización de este trabajo no hubiera podido ser posible sin la colaboración de muchas personas que, de una manera u otra, me han aportado sus conocimientos y brindado su apoyo.

En primer lugar, quiero dar mi más profundo agradecimiento a mi asesor de tesis, el M. en I. Oscar Yañez Suárez, quien me ha proporcionado gran cantidad de los conocimientos que he empleado durante el desarrollo de este trabajo además de agradecer su paciente revisión del texto presentado.

El equipo de la sección de Electroencefalografía del Instituto Nacional de Rehabilitación en la ciudad de México quienes participaron de manera activa en la tarea de diagnóstico de trastorno por déficit de atención con hiperactividad, he de agradecer su aportación de conocimientos médicos necesarios para el desarrollo del proyecto.

Agradecer igualmente a las instituciones que han apoyado parcialmente este trabajo: al Consejo Nacional de Ciencia y Tecnología CONACyT por la beca otorgada para realizar estudios de Posgrado y a la Universidad Autónoma Metropolitana.

Y finalmente, aunque no tengan el más mínimo interés en nada de lo que en este trabajo se presenta, quiero agradecer a toda mi familia compañeros y amigos, que, con su grata compañía durante todo este tiempo, me han proporcionado esos momentos de ocio y descanzo necesarios para finalizar con éxito un duro trabajo como lo es éste. 
A las mujeres de mi vida...

Mi madre Laura, por su apoyo incondicional gracias por haberme enseñado a ser un hombre.

Mi esposa Claudia, por sus consejos y eterna compañia gracias por enseñarme a disfrutar la vida.

Mis hermanas Selma y Viridiana, por soportarme día a día gracias por el tiempo robado.

A mis ejemplos a seguir. . .

Mi padre Federico, por su incansable fortaleza gracias por brindrame, junto a mi madre, la oportunidad de superarme.

Mi hermano Paco, por su original forma de ver el mundo gracias por hacerme querer ser mejor cada día 


\section{Índice general}

Resumen 1

Abstract 2

Agradecimientos 3

Dedicatoria 4

\begin{tabular}{ll}
\hline Introducción & 8
\end{tabular}

1. Motivación 9

1.1. Justificación . . . . . . . . . . . . . . . . . . . . . . . . 10

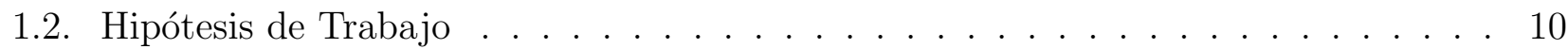

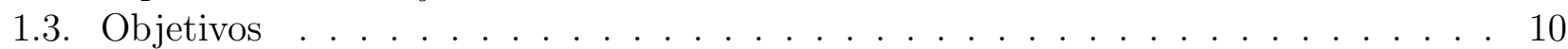

1.3.1. Objetivo general . . . . . . . . . . . . . . . . . 10

1.3.2. Objetivos particulares $\ldots \ldots \ldots \ldots \ldots \ldots \ldots \ldots \ldots$

1.4. Estructura de la tesis $\ldots \ldots \ldots \ldots \ldots \ldots \ldots$

II Antecedentes 14

2. Trastornos por Déficit de Atención en la infancia 15

2.1. Hiperactividad y Disfunción Cerebral Mínima . . . . . . . . . . . . . . 16

2.2. El déficit de atención $\ldots \ldots \ldots \ldots \ldots \ldots \ldots$

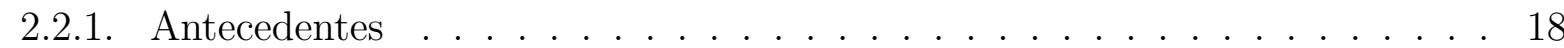

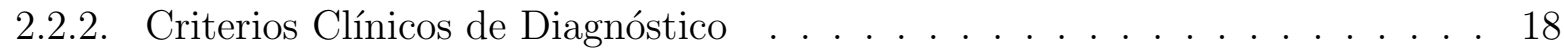

2.3. El papel de los lóbulos frontales $\ldots \ldots \ldots \ldots \ldots \ldots . \ldots \ldots 22$

$\begin{array}{ll}3 . \text { Conceptos generales del reconocimiento de patrones } & 24\end{array}$

3.1. Esquema general de un sistema de RP $\ldots \ldots \ldots \ldots \ldots \ldots \ldots . \ldots . \ldots . \ldots 24$

3.1.1. Bloque de adquisición $\ldots \ldots \ldots \ldots \ldots \ldots \ldots \ldots \ldots \ldots$

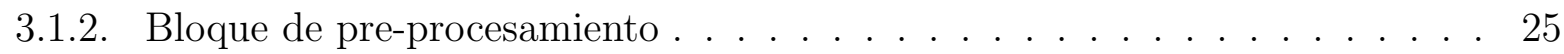

3.1.3. Bloque de extracción de característcas $\ldots \ldots \ldots \ldots \ldots \ldots .25$

3.1.4. Bloque de aprendizaje $\ldots \ldots \ldots \ldots \ldots \ldots \ldots \ldots \ldots \ldots$

3.1.5. Bloque de clasificación $\ldots \ldots \ldots \ldots \ldots \ldots \ldots \ldots \ldots \ldots$

3.2. Aproximación estadística al RP $\ldots \ldots \ldots \ldots \ldots \ldots \ldots \ldots \ldots \ldots$

3.3. Clasificador probabilístico: Regla de Bayes $\ldots \ldots \ldots \ldots \ldots \ldots \ldots .27$

3.4. Estimación paramétrica y no paramétrica $\ldots \ldots \ldots \ldots \ldots \ldots \ldots 28$ 
3.5. Clasificadores basados en distancias . . . . . . . . . . . . . . . . . . . . . . . . 29

3.5.1. El clasificador de los $k$ vecinos mas cercanos . . . . . . . . . . . . . . . 29

3.6. Segmentación/Clasificación mediante análisis cluster . . . . . . . . . . . . . . 31

3.6.1. Métricas de distancia y similitud . . . . . . . . . . . . . . . 31

3.6.2. Clusters no jerárquicos . . . . . . . . . . . . . . . 32

4. Los Mapas Auto-Organizados (SOM)

4.1. Introducción al mapa auto-organizado. . . . . . . . . . . . . . . . . . . . 35

4.2. Arquitectura del mapa auto-organizado . . . . . . . . . . . . . . . . . . 35

4.2.1. Función de vecindad . . . . . . . . . . . . . . . . . . 37

4.3. El Algoritmo de aprendizaje del mapa auto-organizado . . . . . . . . . . . . . . . 38

4.3.1. Mapas auto-organizados como clasificadores . . . . . . . . . . . . 40

III Metodología 41

5. Bosquejo general

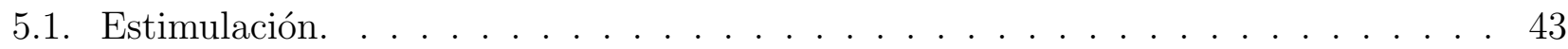

5.2. Adquisición e Instrumentación. . . . . . . . . . . . . . . . . . . 43

5.3. Condiciones de registro $\ldots \ldots \ldots \ldots$. . . . . . . . . . . . . . . . . 44

5.4. Pre-Procesamiento. . . . . . . . . . . . . . . . . . . . . 45

5.4.1. Filtrado . . . . . . . . . . . . . . . . . . . . 45

5.4.2. Segmentación. . . . . . . . . . . . . . . . . . . . 45

5.5. Extracción de características inmersas en las señales Electroencefalográficas. . . . 46

5.5.1. Rasgos Frecuenciales . . . . . . . . . . . . . . . . . . 46 46

5.5.2. Rasgos Wavelet . . . . . . . . . . . . . . . . . . . . 48

$\begin{array}{ll}\text { 6. Arquitectura de análisis } & \mathbf{5 0}\end{array}$

6.1. Proceso de validación del mapa clasificador . . . . . . . . . . . . . . . . . . 52

6.2. Procesamiento del mapa entrenado . . . . . . . . . . . . . . . . . . . . 52

6.3. Interpretación del mapa final . . . . . . . . . . . . . . . . . . . . . . . 55

IV Experimentos, Resultados y Discusión 57

$\begin{array}{ll}\text { 7. Validación del mapa clasificador } & 58\end{array}$

7.1. Resultados del proceso de validación cruzada . . . . . . . . . . . . . . . . . . 59

\begin{tabular}{lll}
\hline 8. Sistema de detección $\left(S O M_{f}\right)$ & $\mathbf{6 4}$
\end{tabular}

8.1. Mapas SOM multicapa como clasificadores . . . . . . . . . . . . . . . . . . 64

8.1.1. Capa I: Mapas por onda cerebral $S O M_{\theta}, S O M_{\alpha}$ y $S O M_{\beta}$. . . . . . . . . . 65

8.1.2. Capa II: Combinación en información de ritmos cerebrales . . . . . . . . . 68

$\begin{array}{ll}9 . & \mathbf{7 0}\end{array}$

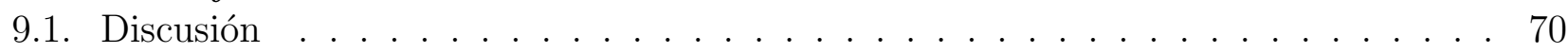

9.2. Conclusiones finales del trabajo . . . . . . . . . . . . . . . . 71

9.3. Trabajo futuro y líneas de investigación abiertas $\ldots \ldots \ldots$. . . . . . . . . 72 


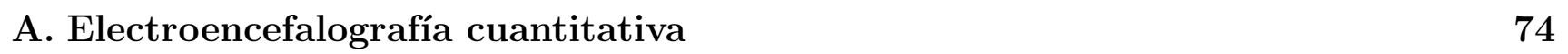

A.1. Principios basicos del EEG . . . . . . . . . . . . . . . . . . . . . . . . 74

A.1.1. Fuentes sinápticas del EEG . . . . . . . . . . . . . . . . . . . . 75

A.1.2. Fuentes neuronales intrínsecas del EEG . . . . . . . . . . . . . . . . . . . . 76

A.1.3. Generadores corticales del EEG . . . . . . . . . . . . . . . . . . . . . . 77

A.1.4. Potenciales de superficie y dendríticos postsinápticos . . . . . . . . . . . 77

A.2. Sistema Internacional de Colocación de Electrodos 10/20 para registro de EEG . . 79

\begin{tabular}{lr}
\hline B. Publicaciones & 83
\end{tabular}

\begin{tabular}{lr}
\hline Bibliografía & 88 \\
\hline
\end{tabular} 


\section{Parte I}

\section{Introducción}




\section{Capítulo 1}

\section{Motivación}

Las mentes son como los paracaídas: sólo funcionan cuando están abiertas.

THOMAS R. DEWAR

El trabajo que se presenta en esta tesis es el resultado de un año de investigación. La línea de trabajo que se ha seguido surge cuando, al haber estado trabajando en proyectos relacionados con el procesamiento y análisis de señales electroencefalográficas asi como de reconocimiento de patrones, se presenta el reto de abordar el problema actual concerniente al diagnóstico de desórdenes neuropsicológicos como lo es el trastorno por déficit de atención con hiperactividad.

La meta primordial era poder brindar una herramienta objetiva adecuada de soporte al diagnóstico de esta patología a partir de un análisis de búsqueda de patrones rasgos o características inmersas en un conjunto de registros electroencefalográficos obtenidos bajo ciertos protocolos de adquisición y estimulación, ésto, con el único fin de ayudar al experto humano clínico durante su tarea diagnóstica.

La tarea se adivinaba verdaderamente dificil, principalmente, por que queríamos implementar una metodología que fuera capaz de ayudar al especialista clínico en el diagnóstico adecuado de dicha enfermedad con un enfoque cuantitativo y en segundo plano, por la necesidad de lograr que el soporte brindado fuera automatizado.

El interés, sin embargo, era demasiado alto, debido a que cualquier mejora, por mínima que esta fuera, respecto a la precisión diagnóstica podía suponer un enorme beneficio para cada uno de los pacientes que presentan este tipo de desórdenes neuropsicológicos. Pronto pudimos darnos cuenta de los pocos trabajos de investigación que habían abordado el problema como tal y por ende, de lo poco que se había podido avanzar en los últimos años en el mejoramiento diagnóstico de estas condiciones, que actualmete se lleva a cabo bajo enfoques puramente cualitativos.

Para poder abordar este proyecto se creó un equipo de trabajo en el que junto con investigadores del Laboratorio de Investigación en NeuroImagenología (LINI) de la Universidad Autonóma Metropolitana, Unidad Iztapalapa (UAM-I), participaron activamente doctores y psicólogos de la sección de Electroencefalografía del Instituto Nacional de Rehabilitación (INR) en la ciudad de México, permitiéndonos el acceso contínuo a la base de datos de sus pacientes referidos para evaluación psicológica. 


\subsection{Justificación}

El electroencefalograma (EEG), continúa siendo una herramienta atractiva en la práctica clínica debido a que proporciona medidas bioeléctricas objetivas de la actividad continua del cerebro, a su no invasividad y a su descripción en tiempo real el cual ha sido utilizado con éxito en el diagnóstico de desórdenes como la epilepsia y esquizofrenia, entre otras, pudiendo también jugar un papel importante en el diagnóstico del Trastorno por Déficit de Atención con Hiperactividad (TDAH).

Debido a que en la actualidad existen disponibles muy pocos procedimientos objetivos de evaluación y diagnóstico clínico para estas condiciones y teniendo en cuenta que los trastornos neuropsicológicos tales como el TDAH se consideran como resultado de alguna disfunción del Sistema Nervioso Central (SNC) [1] y si además se reconoce que el electroencefalograma nos proporciona una medida directa y contínua del funcionamiento eléctrico cerebral entonces, nos atrevamos a pensar que estos registros podrían utilizarse para generar una herramienta adecuada que permita evaluar objetivamente estos trastornos para de esta forma lograr su adecuado diagnóstico y con ello su óptimo tratamiento teniendo como meta a futuro, el desarrollo de técnicas de rehabilitación que compensen su estado.

\subsection{Hipótesis de Trabajo}

La actividad eléctrica cerebral proveniente de los registros electroencefalográficos puede ser ocupada como base para el desarrollo de una herramienta cuantitativa de soporte al diagnóstico del Trastorno por Déficit de Atención con Hiperactividad.

\subsection{Objetivos}

El objetivo último de un soporte al diagnóstico de cualquier tipo de patología es servir de ayuda o apoyo al experto clínico para mejorar su rendimiento o eficiencia diagnóstica.

\subsubsection{Objetivo general}

Determinar conjuntos de rasgos que sean capaces de describir la información procedente de los registros electroencefalográficos para su posterior análisis mediante las redes auto-organizadas de Kohonen (SOM). Con dicho análisis se busca generar mapas clasificadores que permitan apoyar al diagnóstico mediante la categorización objetiva del trastorno por déficit de atención con hiperactividad.

\subsubsection{Objetivos particulares}

1. Evaluar las modificaciones en las bandas espectrales de los ritmos cerebrales por la fotoestimulación a partir, de los registros electroencefalográficos adquiridos.

2. Determinar rasgos tiempo-frecuencia que sean capaces de describir adecuadamente la actividad eléctrica contínua del cerebro representada por el Electroencefalograma durante dichas pruebas. 
3. Mediante un modelo basado en mapas auto-organizados, determinar si existen patrones que relacionen a los sujetos diagnosticados con TDAH, teniendo principal interes de poderlos clasificar de forma automatizada, realizando para ello una búsqueda de características o rasgos comunes, para los sujetos con esta enfermedad, que la red auto-organizada sea capaz de clasificar eficientemente.

4. Comparar los resultados obtenidos respecto a la población patológica, con otra población constituida de sujetos sanos, que serán tomados como referencia durante la determinación de las zonas generadas por el mapa clasificador.

Toda la información utilizada para realizar la investigación presentada en esta tesis, fue tomada de una base de datos de sujetos diagnosticados con y sin trastorno por déficit de atención con hiperactividad en el Instituto Nacional de Rehabilitación.

\subsection{Estructura de la tesis}

El trabajo presentado en esta tesis se ha estructurado en nueve capítulos que conforman cuatro partes, incluida ésta.

- En la Parte II se da una breve reseña sobre los antecedentes concernientes a la investigación presentada en esta tesis.

- El primer capítulo muestra una introducción general a los trastornos por déficit de atención en la infancia, que sirve de base primero, para entender la patología desde un contexto tanto clínico como fisiológico y segundo, para justificar las razones del uso del protocolo de estimulación aplicado durante la adquisición de los registros y el cual es explicado a detalle en la parte III

- Posteriormente en el capítulo 3, de manera muy general se esbozan los conceptos y aspectos más importantes que constituyen al reconocimiento de patrones, que servirá para poder presentar en los siguientes capítulos, tanto el procedimiento de extracción de caracteríisticas de la actividad electrica cerebral como las técnicas de clasificación que serán ocupadas para implementar la arquitectura y el análisis de los datos explicados en la parte III de esta tesis.

- Finalmente en el capítulo 4, se presenta la técnica específica con la que se analizaron los datos obtenidos después de la extracción de características, los mapas auto-organizados de Kohonen, abordando desde el surgimiento de éstos hasta la forma en que trabajan y la manera de interpretarlos.

- La Parte III expone en su primer capítulo, los pasos y las técnicas empleadas para implementar el sistema de soporte al diagnóstico de TDAH que se propone en esta tesis. Básicamente se explican los protocolos de estimulación, adquisición y procesamiento, detallando las condiciones deseables de los sujetos a estudiar, así como las aproximaciones matemáticas empleadas para llevar a cabo la extracción de características de la actividad eléctrica cerebral de los sujetos estudiados y en su segundo capítulo, se presenta la arquitectura de análisis de los rasgos propuesta para el diseño del mapa clasificador final como sistema de soporte al diagnóstico de TDAH. 
- Los dos primeros capítulos de la Parte IV muestran a detalle primeramente, el proceso con el cual se llevó a cabo la validación del soporte propuesto y en segundo plano, los resultados obtenidos después de aplicar lo mencionado en partes anteriores, detallando los experimentos y procesamientos realizados durante los procesos de tratamiento digital, validación e interpretación del soporte.

- Finalmente, en el último capítulo de la Parte IV se expone la discusión realizada sobre cada uno de los resultados obtenidos a lo largo del proyecto de investigación realizado para posteriormente discutir las conclusiones finales de todo el trabajo presentando primeramente, las posibles líneas de investigación que quedan abiertas a futuros proyectos relacionados con la presente tesis así como las posibles limitaciones encontradas para el soporte propuesto.

El contenido general de las partes y capítulos mencionadas, se resume en la figura 1.1

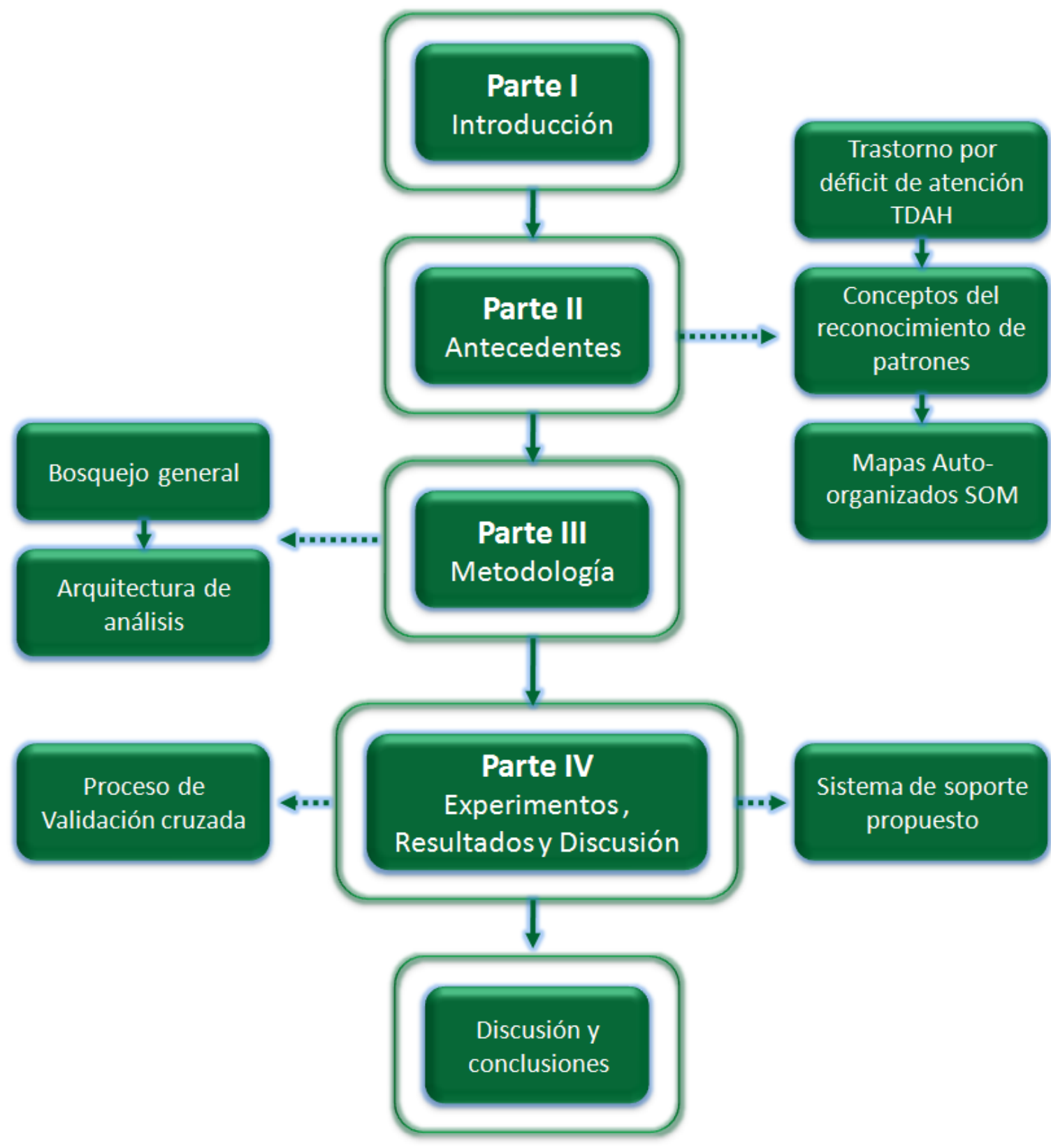

Figura 1.1

Diagrama a bloques de la estructura que conforma a este trabajo. 
Por otro lado, en el Anexo A se muestra lo relacionado con las técnicas electroencefalográficas, presentando desde los principios fisiológicos relacionados con la electroencefalografía hasta la definición general del sistema internacional de colocación de electrodos 10/20 que fue utilizado durante la adquisición de cada uno de los registros.

Mientras que en el Anexo B, se decidió colocar el articulo generado durante esta investigación el cual, fue presentado en la conferencia anual internacional de la sociedad de ingeniería en medicina y biología (EMBC'10) celebrada en Buenos Aires, Argentina del 31 de Agosto al 4 de Septiembre de 2010. 


\section{Parte II}

Antecedentes 


\title{
Capítulo 2
}

\section{Trastornos por Déficit de Atención en la infancia}

\author{
Dale a una persona un pez y le alimentarás por un día. \\ Enseña a una persona a pescar y le alimentarás toda su vida. \\ PROVERBIO CHINO
}

El problema de la inatención en el ámbito escolar es relativamente frecuente en los niños. Los trastornos por déficit de atención propiamente diagnósticados, ocurren entre el 3 y el $10 \%$ de la población infantil en edad escolar dependiendo siempre por supuesto, de los criterios diagnósticos empleados y del rigor metodológico de los investigadores. Las estimaciones de las que se informa con más frecuencia están alrededor del $5 \%$, lo que lleva a pensar que aproximadamente un niño por clase tendría esta patología, además, resulta interesante saber que en la sociedad americana el $50 \%$ de los niños que son remitidos para atención clínica presenta déficit atencional y/o hiperactividad [2].

El trastorno o desorden de déficit de atención con o sin hiperactividad (conocido en el pasado como síndrome hipercinético), es de especial preocupación tanto para padres y educadores como para psicólogos, psiquiatras e investigadores. Lo típico de un niño hiperactivo es que esté en contínuo movimiento, que sólo se pueda concentrar durante lapsos cortos de tiempo, que actúe y hable dejándose llevar por sus impulsos, que sea impaciente y se desconcentre fácilmente.

Después de algunos años el interes se dirigió hacia el déficit atencional, cuyas características princiales son: la presencia de dificultades para alcanzar la concentración durante la realización de alguna actividad, pasar de una a otra sin concluirlas y sobre todo, presentar problemas de distractibilidad, impulsividad, torpeza social e inadecuada resolución de problemas.

El déficit atencional mostrado por los niños hiperactivos ha convertido al trastorno por déficit de atención en una cuestión de máximo interés dentro de la psicopatología infantil.

Este capítulo aborda la hiperactividad como un síndrome, mostrando al déficit atencional como síntoma principal, permitiendo entrar de lleno al trastorno por déficit de atención, partiendo de las bases neuropsicológicas de la enfermedad hasta su asociación con otras alteraciones, abordando también, su caracterización por las clasificaciones diagnósticas. 


\subsection{Hiperactividad y Disfunción Cerebral Mínima}

Resulta un tanto ambiguo el hablar del niño hiperactivo ya que puede referirse a un niño con hiperactividad, que es el síntoma que el niño presenta por su excesiva inquietud o movilidad inapropiada y no dirigida intencionalmente o bien, puede referirse al síndrome de hiperactividad, en el que la hiperactividad no es siempre el indicador principal, siendo preferible entender por niño hiperactivo a la segunda en su sentido más amplio.

Al no existir una definición precisa, el síndrome de hiperactividad se ha descrito como un conjunto de síntomas de intranquilidad motora, de impulsividad, de reducida capacidad atencional, de dificultades específicas de aprendizaje (mostrando en particular a la dislexia) y de inestabilidad emocional. La disfunción cerebral mínima (DCM) por otro lado, alude al mismo conjunto de síntomas que el síndrome de hiperactividad, siendo este último el trastorno observado de conducta o el estado mental basado en un funcionamiento anormal del cerebro, postulando, que la conducta hiperactiva se deriva de un daño cerebral mínimo, es decir, se tendió a creer que el daño cerebral causaba tal conducta sin embargo, al no poder inferir daño cerebral en todos los casos de conducta hiperactiva se recomendó reemplazar el término daño, por disfunción [3].

Cuando un niño no presenta daño neurológico y además sus puntuaciones en capacidad intelectual son normales, a pesar de la no delimitada definición del síndrome hipercinético o DCM, se considera muy frecuentemente que posee dicho sindrome si muestra ciertas dificultades de aprendizaje, no rinde como debiera en las tareas escolares y se distrae con facilidad. La anomalía clínica más común en el EEG es la de un exceso en las ondas lentas, primordialmente en la onda theta, actividad que suele encontrarse en niños normales más pequeños, apoyando la hipótesis de un retraso madurativo del sistema nerviso central (SNC) en los niños con síndrome hipercinético.

\subsection{El déficit de atención}

El trastorno por déficit de atención, es una de las condiciones más comunes de la niñez y la adolescencia (6 - 17 años), caracterizada por niveles variables de la hiperactividad, impulsividad (pobre control de impulsos, indica la tendencia a responder a los estímulos rápidamente y sin tener en cuenta las alternativas) e inatención (incapacidad para persistir en la ejecución de una tarea más allá del desinterés o aburrimiento, independientemente de la ausencia o presencia de estímulos distractores), que puede ir cambiando con el desarrollo del sujeto durante la edad adulta. Este desorden interfiere con muchas áreas de desarrollo normal y funcionamiento en la vida de un niño y si no es tratado, predispone a deteriorar de modo significativo el funcionamiento cognitivo y de conducta generando problemas sociales y psiquiátricos [4].

Actualmente se cuestiona si el trastorno por déficit de atención con hiperactividad es propiamente una dificultad específica de aprendizaje o se trata de un trastorno relacionado, a esto se dice, que se atribuye DCM a niños de inteligencia general próxima a la media, en la media o superior a ésta, con ciertas dificultades de aprendizaje o de conducta las cuales se asocian con desviaciones funcionales del SNC. Estas desviaciones pueden manifestarse a través de varias combinaciones de déficit en la percepción, conceptualización, lenguaje, memoria y control de la atención, impulso o función motora como se intenta reflejar en el esquema siguiente: 


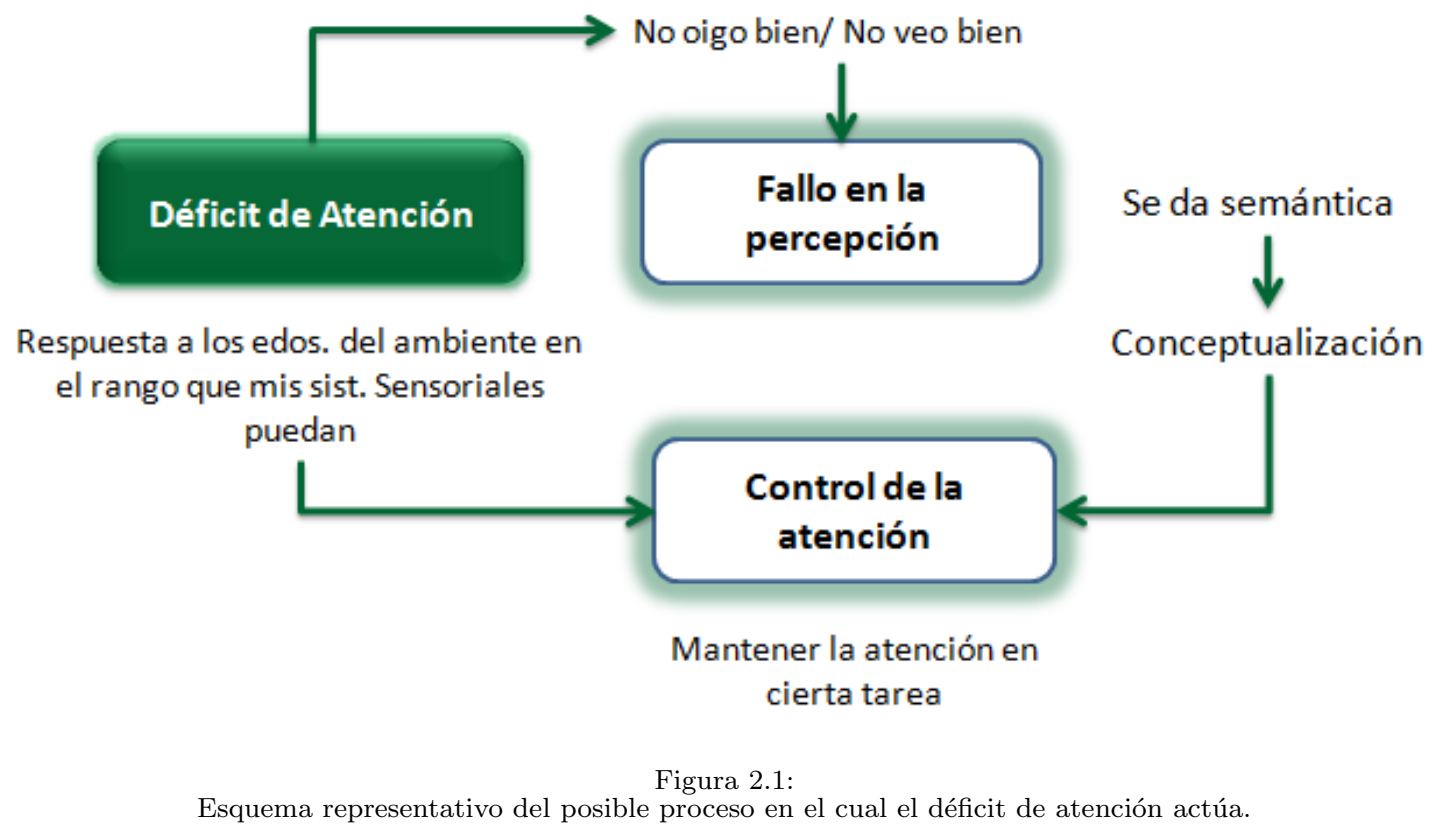

Las características citadas con mayor frecuencia por los expertos al describir la DCM son:

1. Hiperactividad e Impulsividad

2. Déficit perceptivo-motor

3. Labilidad emocional

4. Déficit de coordinación en general

5. Trastornos de atención, de memoria y de pensamiento

6. Dificultades especificas de aprendizaje: de lectura, aritmética, escritura y deletreo

7. Trastornos del habla y de la audición Irregularidades en el EEG

A partir de realizar esta caracterización de la DCM, se establecen las tres líneas de investigación que presentaron mayor interes:

1. Dificultades de aprendizaje: problemas de lectura, aritmética y escritura como la dislexia, discalculia y disgrafía.

2. Interés principal en el estudio de niños que presentan hiperactividad, distractibilidad e impulsividad. Surge la primera clasificación diagnóstica del DSM-II] como reacción hipercinética de la infancia. ${ }^{2}$

\footnotetext{
${ }^{1}$ Manual diagnóstico y estadístico de desordenes mentales elaborada por American Psychiatric Association, APA, en 1968

${ }^{2}$ En la edición de 1980, el DSM-III enfatizó la distractibilidad (pobre concentración, indica incapacidad para atender selectivamente a los estímulos relevantes o apropiados de una situación) como síntoma primario en relacióon con los otros dos y modificó la denominación por trastorno por déficit de atención, TDA. La revisión de 1987, el DSM-III-R cambió a su vez, la denominación anterior por la de trastorno por déficit de atención con hiperactividad, TDAH, manteniéendo la distractibilidad como cuestión primaria pero además, considerando a la hiperactividad como un factor importante
} 
3. Problemas emocionales y sociales; el centro de interés crítico en las investigaciones bajo esta dirección consistía en saber si los problemas socioemocionales de pacientes causaban las dificultades académicas o más bien eran su consecuencia.

\subsubsection{Antecedentes}

Los pacientes que han sido diagnosticados con TDAH manifiestan déficits, equivalentes a los pacientes que presentan lesión en los lóbulos frontales, en tareas neuropsicológicas tal como la atención contínua [5].

Las investigaciones de los últimos años en electroencefalografía han encontrado diferencias consistentes entre niños con y sin TDA basicamente, la actividad tónica cortical (o de base) aparece más lenta en niños con la patología, encontrando que éstos presentan ritmos EEG tónicos menos activados que los sujetos control, es decir, los niños que han sido diagnosticados con TDAH típicamente muestran un exceso de actividad en los ritmos lentos: delta $\delta$ y theta $\theta$ y en contraparte una disminución de la actividad de los ritmos rápidos: alpha $\alpha$ y beta $\beta$; comparándolos con los presentes en individuos normales sujetos a la condición de ojos cerrados [5].

Además, se ha encontrado un porcentaje significativamente mayor de actividad epileptiforme que los niños normales, siendo más evidente durante la hiperventilación y la fotoestimulación, presentándose también anormalidades interhemisféricas como asimetría de potencia, disminución de la coherencia entre las regiones parietales y temporales y aumento de ésta para las regiones frontales y centrales [6].

Resultados de otros investigadores refuerzan el supuesto de que los sujetos con este desorden pueden tener una disminución en el nivel de activación cortical, apoyando la hipótesis de presencia de un retraso en la maduración de sus sistemas neurales frontales [7], es decir, que ante una tarea cognitiva demandante se esperaría un aumento de señales rápidas tal y como ocurre en niños sin este trastorno.

El diagnóstico actual de este desorden neuropsicológico se basa, principalmente, en observaciones del comportamiento del niño como el informado por los padres y/o maestros a través de cuestionarios estandarizados (DSM-III, DSM-III-R y DSM-IV), lo cual puede ser muy subjetivo. La necesidad de un estudio objetivo e independiente para este desorden resulta ser de mucho interes, para así, poder determinar si existe alguna disfunción de estos individuos presente en el sistema nervioso central.

Una posible medida objetiva para soportar el diagnóstico de TDAH puede obtenerse de la electroencefalografía cuantitativa.

\subsubsection{Criterios Clínicos de Diagnóstico}

Conte, 1991, advierte de los diferentes sentidos en los que se ha empleado la palabra atención, ya que, por una parte, atención puede significar procesos muy diversos según las teorías atencionales, como estado de alerta, selección o esfuerzo. En cambio, si se habla de niños con 
TDA e hiperactividad, el concepto de atención es considerablemente más complicado, de hecho, la atención es sólo un aspecto de la compleja sintomatología del TDAH.

\section{Escalas de Comportamiento Infantil (ECI)}

Entre las aproximaciones conductuales a la evaluación del TDAH se halla el uso de escalas de calificación de conductas o listas de chequeo (estrategías de evaluación indirectas que sirven para evaluar ciertas dimensiones de la conducta de los niños), en las que se pide a los informantes (padres, maestros, compañeros e incluso el propio sujeto) que califiquen ciertas conductas del niño observadas en el pasado.

Dichas evaluaciones se contestan en muy poco tiempo y muy fácilmente, pudiéndose obtener datos de muy diversas situaciones en las que el niño se manifiesta de modo más espontáneo y habitual.

Los métodos de cuantificación de los datos, como el análisis factorial, han permitido identificar conductas intercorrelacionadas que se pueden interpretar formando una dimensión conductual de interés clínico. La medición de estos desórdenes mentales a través de escalas de calificación de conductas ha sido ampliamente utilizada en investigación y en sistemas de aplicación clínica.

Entre las medidas de atención se hallan; las escalas de calificación de conducta, pruebas psicométricas y medidas de laboratorio, así como los sitemas de observación directa. Actualmente, la evaluación de los problemas de atención resulta muy complejo y es sensato decir que no es posible evaluarla con una sola prueba.

Las limitaciones de las escalas pueden provenir de los propios datos normativos y de su elaboración, de la subjetividad del calificador, así como también de la ambiguedad de la interpretación de los items. A pesar de tales limitaciones inherentes a las escalas, es indudable su utilidad, tanto en el ámbito clínico como en el investigador, para evaluar el TDAH.

\section{Criterios diagnósticos del DSM-III}

Los criterios diagnósticos del DSM-III han influido de forma significativa en las definiciones actuales del TDAH, estos criterios se basan en los resultados obtenidos en la práctica, pidiéndole a los profesionales clínicos los síntomas en los que se apoyan para emitir diagnósticos sobre diversos trastornos.

El DSM-III de 1980 definió dos tipos de TDA, el que presenta hiperactividad y el que carece de ella, por otro lado el aspecto atencional de este trastorno implicaba dos clases de síntomas: inatención e impulsividad.

Ahora bien, al mirar las características del trastorno de atención siguiendo los criterios del DSM-III sobre el TDA mostrados en la tabla 2.1, se aprecia que muchas de las características

seleccionadas tienen muy poco que ver con el concepto de atención discutido en las teorías de la 
atención.

Tabla 2. 1

Criterios del DSM-III: Criterios diagnósticos para el trastorno por déficit de atención.

\begin{tabular}{|c|c|}
\hline A & Inatención. $\mathrm{Al}$ menos tres de los siguientes síntomas \\
\hline 1 & No termina las cosas que comienza \\
\hline 2 & A menudo no parece escuchar \\
\hline 3 & Se distrae con facilidad \\
\hline 4 & Tiene dificultades para concentrarse en el trabajo escolar o en tareas que requieren atención sostenida \\
\hline 5 & Tiene dificultades para concentrarse en el juego \\
\hline B & Impulsividad. Al menos tres de los siguientes síntomas \\
\hline 1 & A menudo actúa antes de pensar \\
\hline 2 & Cambia con excesiva frecuencia de una actividad a otra \\
\hline 3 & Tiene dificultades para organizarse en el trabejo \\
\hline 4 & Necesita supervisión constante \\
\hline 5 & Frecuentemente levanta mucho la voz en clase \\
\hline 6 & Le cuesta guardar turno en los juegos o en situaciones de grupo \\
\hline $\mathrm{C}$ & Hiperactividad. Al menos dos de los siguientes síntomas \\
\hline 1 & Corre en exceso de un lado para el otro, o se sube a los muebles \\
\hline 2 & Le cuesta mucho permanecer en un sitio, o se mueve excesivamente \\
\hline 3 & Le cuesta estar sentado \\
\hline 4 & Se mueve excesivamente mientras duerme \\
\hline 5 & Está siempre en marcha, o actúa como movido por un motor \\
\hline
\end{tabular}

$\mathrm{D}$

Edad de comienzo anterior a los siete años.

E Duración de al menos seis meses.

F No debido a esquizofrenia, trastorno afectivo ni a retraso mental severo o profundo.

\section{Criterios diagnósticos del DSM-III-R}

En la revisión de 1987, se eliminó la distinción entre TDA con y sin hiperactividad, debido a los pocos apoyos empíricos con que contaba tal distinción.

En su lugar, se decidió tratar el trastorno de atención como una entidad unitaria, denominada trastorno por déficit de atención con hiperactividad, TDAH, que presentaba un conjunto de manifestaciones diferentes, mostradas en la tabla 2.2, refiriendose unos a inatención, otros a conducta impulsiva y otros a sobreactividad.

Sin embargo existe un desacuerdo respecto a la naturaleza fundamental del TDAH, mientras los datos sugieren que los componentes de atención e hiperactividad no están significativamente 
correlacionados, el DSM-III-R propone una visión unidimensional del trastorno. Es más, el componente de impulsividad es dificilmente separable de los de inatención e hiperactividad.

Tabla 2. 2

Criterios del DSM-III-R: Criterios diagnósticos para el trastorno por déficit de atención con hiperactividad.

\begin{tabular}{ll}
\hline A & \multicolumn{1}{c}{$\begin{array}{c}\text { Una alteración de por lo menos seis meses de evolución } \\
\text { con la presencia de por lo menos ocho de los sintomas siguientes }\end{array}$} \\
& \\
\hline 1 & $\begin{array}{l}\text { Inquietud frecuente, que se aprecia por movimientos de manos o pies o por moverse en el asiento } \\
\text { En adolescentes puede estar limitado a sensasiones subjetivas de impaciencia e inquietud }\end{array}$ \\
2 & Dificultad para permanecer sentado cuando la situación lo requiere \\
3 & Fácil distraibilidad por estímulos ajenos a la situación \\
4 & Dificultad para guardar turno en los juegos o situaciones de grupo \\
5 & Frecuencia de respuestas precipitadas antes de que se acaben de formular las preguntas \\
6 & Dificultad para seguir las instrucciones de los demás, no debido a negativismo, o a error densión \\
7 & Dificultad para mantener la atención en tareas o actividades lúdicas \\
8 & Frecuentes cambios de una actividad incompleta a otra \\
9 & Dificultad para jugar con tranquilidad \\
10 & A menudo habla excesivamente, verborrea \\
11 & A menudo interrumpe o se implica en actividades de otros niños \\
12 & A menudo no escucha lo que se le dice \\
13 & A menudo pierde cosas necesarias para una tarea o actividad escolar \\
14 & A menudo practica actividades peligrosas sin evaluar los posibles riesgos
\end{tabular}

$\mathrm{B}$

Comienzo antes de los siete años

C No reúne los criterios para el diagnóstico de trastorno generalizado del desarrollo

El paso de la definición multidimensional que propone el DSM-III a la unidimensional del DSM-III-R implica un aumento en el número de casos diagnósticados como TDAH [8] por lo cual, la superioridad del DSM-III-R frente a su predecesor está muy poco clara además, la detección de falsos positivos no favorece ni a la práctica clínica ni a la investigación.

Lo anterior, puede desorientar al momento de establecer respuestas a tratamientos y seguimientos de pacientes a largo plazo.

\section{Criterios diagnósticos del DSM-IV}

Los criterios que presenta el DSM-IV ofrecen una revisión a los presentados por el DSM-III-R respecto a los trastornos de conducta perturbadores.

En lo que se refiere al TDAH, éste puede reunir algunos criterios de predominio de la inatención, clasificado como del tipo I, o de predominio de la hiperactividad-impulsividad, clasificado como del tipo II, o bien reunir en un tipo combinado los criterios de los dos primeros tipos y clasificarlo como del tipo III.

El contenido de la tabla 2.3 presenta los criterios para la clasificación diagnóstica de estos tres tipos de TDAH, según el DSM-IV. 
Tabla 2.3

Criterios del DSM-IV: Criterios diagnósticos para el trastorno por déficit de atención con hiperactividad.

\begin{tabular}{ll}
\hline \hline & \\
A & O bien (1) o bien (2) \\
\hline & \\
$(1)$ & Inatención \\
$a$ & A menudo falla al prestar adecuada atención a los detalles o comete errores por descuido en sus actividades \\
$b$ & A menudo tiene dificultad para mantener la atención en tareas o actividades lúdicas \\
$c$ & A menudo parece no escuchar lo que se le dice \\
$d$ & A menudo no sigue las instrucciones y falla en acabar las tareas escolares o deberes en el lugar de trabajo \\
& No debido a oposicionismo o a error de comprensión \\
$e$ & A menudo tiene dificultades para organizar tareas y actividades \\
$f$ & A menudo evita o le disgustan mucho tareas que requieren esfuerzo mental sostenido \\
$g$ & A menudo pierde cosas necesarias para una tarea o actividad escolar \\
$h$ & Facil distraibilidad por estímulos ajenos a la situación \\
$i$ & A menudo es olvidadizo para las actividades de cada día
\end{tabular}

(2) Hiperactividad-Impulsividad ${ }^{4}$

Hiperactividad

a Inquietud frecuente, que se aprecia por movimientos de manos o pies, o por moverse en el asiento

$b \quad$ Deja de estar sentado en clase o en otras situaciones en las que se espera que esté sentado

c A menudo corre de un lado a otro o salta excesivamente en situaciones en las que es inapropiado

En adolescentes y adultos puede limitarse a sensaciones subjetivas de inquietud

d Dificultad para jugar o entenderse en actividades de esparcimiento con tranquilidad

Impulsividad

e Frecuencia de respuestas precipitadas antes de que se acaben de formular las preguntas

$f \quad$ A menudo tiene dificultad para esperar en fila o guardar turno en los juegos o situaciones de grupo

$\mathrm{B}$

Comienzo no después de los siete años

C Los síntomas deben darse en dos o más situaciones, por ejemplo, en la escuela y en la casa

D La ateración causa perturbación o deterioro clínicamente significativo en el funcionamiento social, académico u ocupacional

E No ocurre exclusivamente en el curso de un trastorno generalizado del desarrollo, de esquizofrenia u otro trastorno psicótico, y no se explica mejor por un trastorno del humor, un trastorno de ansiedad, un trastorno disociativo, ni un trastorno de personalidad

\section{3. $\quad$ El papel de los lóbulos frontales}

Dentro de la pluralidad funcional de los lóbulos frontales, destacan tres hechos neuroanatómicos que van siendo cada vez mejor conocidos:

1. La riqueza de conexiones aferentes y eferentes con diversas estructuras no especificadas del cerebro: tálamo, región hipotalámica y formación reticular del mesencéfalo

\footnotetext{
${ }^{3} \mathrm{Al}$ menos seis de los síntomas de inatención han persistido como mínimo durante seis meses en un grado mal adaptativo o inconsistente con el nivel de desarrollo

${ }^{4} \mathrm{Al}$ menos cuatro de los síntomas de hiperactividad-impulsividad han persistido como mínimo durante seis meses en un grado mal adaptativo o inconsistente con el nivel de desarrollo
} 
2. Las numerosas vías de comunicación que unen la corteza prefrontal con otras regiones corticales.

3. La corteza prefrontal del hombre posee áreas especializadas en el control de la actividad de ciertos sistemas.

Por lo anterior, los lóbulos frontales participan en la regulación de los procesos de activación que están en la base de la atención voluntaria. La actividad cortical se mantiene en forma óptima como condición básica de toda actividad consciente, es decir, la formación de planes e intenciones capaces de oponerse a la distracción procedente de estímulos irrelevantes. La selectividad de los procesos mentales se altera, y es el principal síntoma, cuando se producen lesiones en las zonas mediales frontales [9].

Recientemente se ha mostrado cómo los niños con TDAH realizan pobremente pruebas que miden en adultos disfunción de los lóbulos frontales [10]. Sus resultados parecen apoyar la hipótesis de algun tipo de disfunción de los lóbulos frontales en éstos niños, sin que esa disfunción represente un deterioro cognitivo generalizado, principalmente por que realizaron normalmente tareas de memoria sensibles a la disfunción de lóbulo parietal.

El estudio neuropsicológico de Fournier [11] permite extraer conclusiones que indican claramente disfunción de lóbulos frontales, en este caso relevándose los niños hiperactivos (mediante el análisis discriminante) muy deficientes en tres subpruebas de la bateria de Luria [12] estrechamente dependientes de la regulación verbal del acto motor, presentándose dicha disfunción muy diferenciada de la disfunción de los niños con epilepsia rolándica.

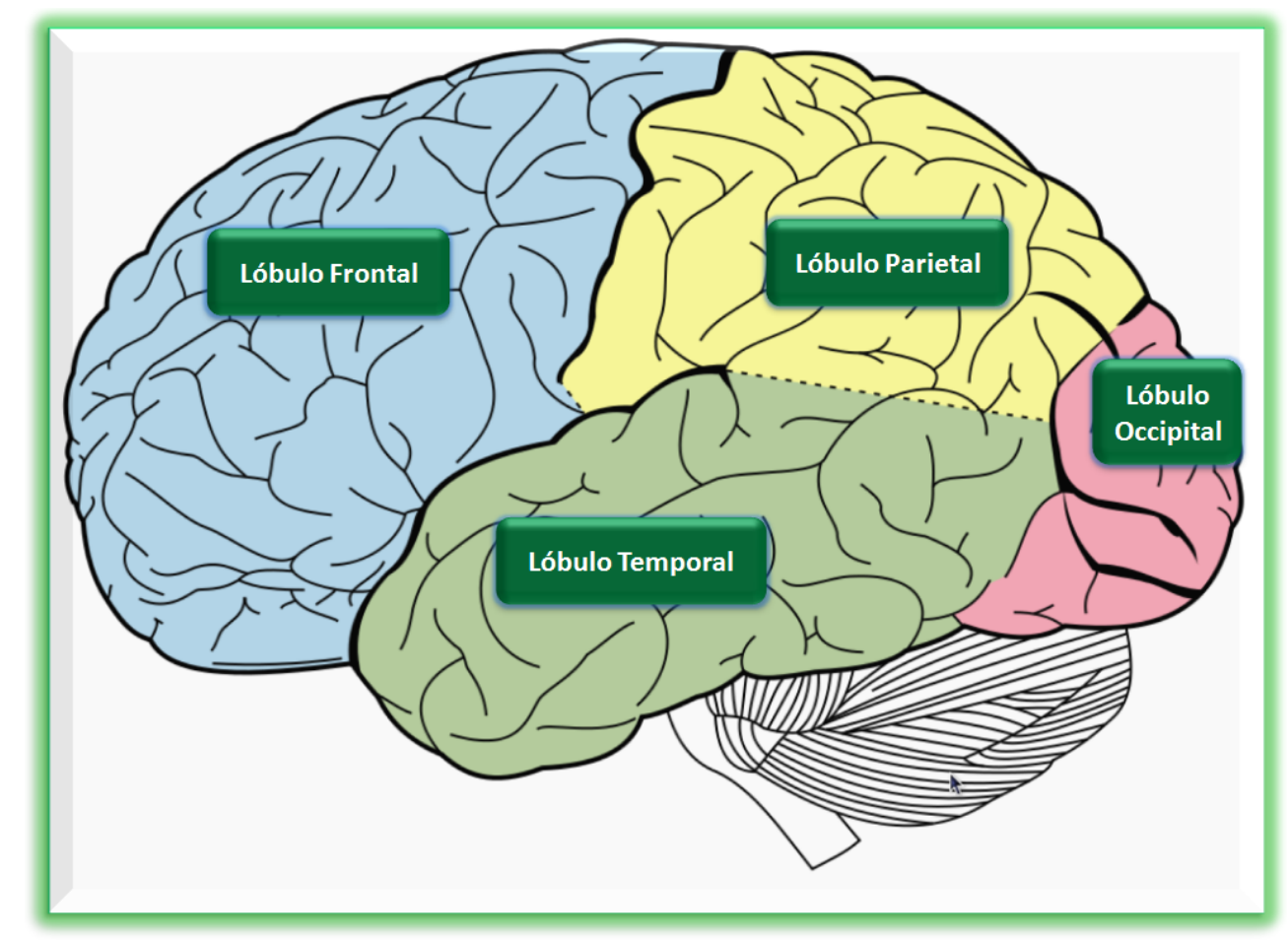

Figura 2.2

Visión lateral de los lóbulos cerebrales. 


\section{Capítulo 3}

\section{Conceptos generales del reconocimiento de patrones}

No basta tener buen ingenio; lo principal es aplicarlo bien.

RENÉ DESCARTES

En este capítulo se presenta una breve revisión de los conceptos básicos del reconocimiento de patrones (RP), haciendo un mayor hincapié en los aspectos más directamente relacionadas con el proyecto de investigación realizado. Basicamente, se presenta la aproximación general en la que se pueden agrupar las distintas técnicas empleadas en el reconocimiento de patrones, la aproximación estadística o geométrica. Se aborda también la regla de Bayes y se introducen además algunos clasificadores de distinta naturaleza que serán utilizados en la elaboración de este trabajo así como las bases teóricas del análisis cluster (análisis de grupos o redes) que será ocupado para la búsqueda de conglomerados y la segmentación de los mismos.

\subsection{Esquema general de un sistema de RP}

Cualquier sistema de reconocimiento de patrones opera funcionalmente primero, mediante un proceso de entrenamiento que conlleva a un aprendizaje maquinal que posteriormente, permite generar una clasificación del objeto que deseamos estudiar; en la figura 3.1 se muestra un modelo genérico para un sistema de reconocimiento de patrones.

Cada uno de las bloques que conforman a dicho sistema tendrá características específicas y determinadas siempre en función del tipo de sistema de reconocimiento que se desee implementar. En los Capítulos 4, 5 y 6 se describe a detalle el conjunto de etapas que conforman al bloque de preprocesamiento, obtención de características y clasificación de las mismas, las cuales están orientadas al diseño de un sistema de soporte al diagnóstico de TDAH a partir únicamente de un conjunto de registros electroencefalográficas adquiridos bajo un protocolo específico de estimulación.

A continuación se describe a manera de bloques y de modo más genérico, las funciones básicas para cada una de las etapas que conforman al modelo genérico de reconocimiento de patrones mostrado en la figura 3.1. 


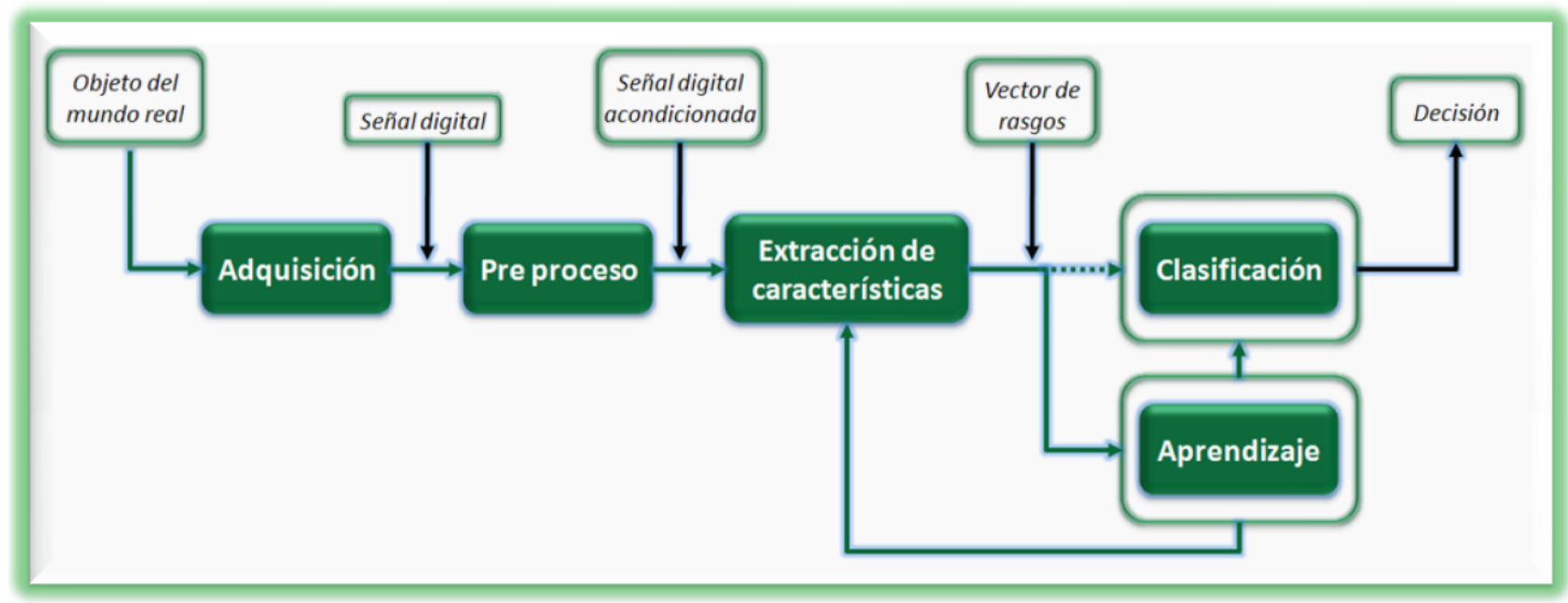

Figura 3.1:

Representación en bloques del modelo genérico para un sistema de reconocimiento de patrones.

\subsubsection{Bloque de adquisición}

En este primer bloque se adquiere un objeto del mundo real mediante los sensores físicos apropiados, por ejemplo, un equipo de imagenología para la extracción de imágenes médicas, un micrófono para capturar la voz o en éste caso, un equipo electroencefalográfico que permita registrar la actividad eléctrica cerebral humana. En muchos casos esta información se presenta en forma de señal analógica, la cual puede ser preprocesada para mejorar su calidad o para aislar la parte útil de la misma. Una posterior digitalización de la señal permite que ésta pueda ser tratada por un computadora, ya sea para almacenarla en algún tipo de soporte permanente o bien, para utilizarla directamente como entrada en el bloque posterior. Se dice que la señal obtenida pertenece al espacio de representación primario.

\subsubsection{Bloque de pre-procesamiento}

La función del bloque de preprocesamiento, también llamado de tratamiento digital de la señal, consiste, básicamente, en realzar y acondicionar el patrón de interés respecto al resto de la señal original. Para esta investigación en particular, al tratarse de señales cerebrales, las operaciones habituales de este bloque consisten en la sustracción digital, el filtrado y la segmentación. Además, cuando el módulo de preprocesamiento opera en modo de entrenamiento, se realizará el etiquetado del patrón para permitir su posterior identificación. Buena parte de estas operaciones son materia de un campo de trabajo particular como es el del tratamiento digital de señales, sobre el que existe extensa literatura [13].

\subsubsection{Bloque de extracción de característcas}

Las características de un objeto son el resultado de aplicar un cierto conjunto de funciones matemáticas sobre las muestras obtenidas del objeto (señal digital acondicionada), que permiten poder distinguir la clase a la cual pertenecen y que serán utilizadas como entrada al clasificador. El objetivo de este bloque es determinar el conjunto de características que mejor representen al objeto de entrada, es decir, aquellas que minimicen las diferencias intraclase y maximicen las diferencias interclase. Durante el entrenamiento, es frecuente realizar diversas iteraciones sobre 
este bloque para tratar de optimizar la eficiencia del sistema.

La obtención de características es la parte menos sistemática del proceso y tiene una gran dependencia del tipo de tarea que se desea realizar. Normalmente, la experiencia y la intuición del diseñador del sistema será de gran importancia durante la resolución exitosa de esta etapa. Se consideran tres tareas fundamentales en el proceso de obtención de características: primeramente la extracción, que hace referencia a la transformación de la señal del dominio primario a un dominio más adecuado para tratar el problema; la selección, con la que se escogen aquellas características que aportan información valiosa para la identificación del objeto, eliminando aquellas con información no discriminante o redundante; y por último la reducción del número de características, que contribuirá enormemente en la redución del costo computacional final.

\subsubsection{Bloque de aprendizaje}

El clasificador es entrenado de modo tal que intente particionar el espacio de características generado por el bloque anterior, con el objetivo principal de minimizar el error de clasificación entre los prototipos del conjunto de validación. La retroalimentación hacia los bloques anteriores permite al diseñador del sistema escoger, entre las distintas técnicas probadas, aquella que presente un mejor rendimiento, atendiendo a los resultados obtenidos al clasificar el conjunto de validación.

\subsubsection{Bloque de clasificación}

En este bloque, el clasificador toma el vector de rasgos (resultado del bloque de extracción de características) extraído del patrón de entrada y le asigna la clase con la que se encuentra una mayor similitud, de acuerdo al modelo inducido durante el entrenamiento. En ocasiones la desición de salida del clasificador no se limita a la clase más similar y puede ofrecer múltiples hipótesis conteniendo una etiqueta de clase, hipótesis, y el nivel de probabilidad asignado a ésta.

\subsection{Aproximación estadística al RP}

En el reconocimiento estadístico de patrones, un patrón se representa por un conjunto de $k$ características, rasgos o atributos en forma de un vector de dimensión $k$, esperando que los patrones de una misma clase se agrupen en regiones del espacio determinadas por las densidades dependientes de cada clase denominadas densidades condicionales de clase, obtenidas a partir de un conjunto de muestras de entrenamiento. Esto posibilita la existencia de metodologías estadísticas que permiten la clasificación de nuevos patrones, lo que constituye el objetivo último de cualquier sistema de reconocimiento.

Existen diversas estrategias a la hora de diseñar un clasificador estadístico, siempre en función del tipo de información disponible sobre las densidades condicionales de clase. Si se dispone de densidades condicionales de clase completamente conocidas, se adopta la regla de decisión de Bayes óptima. Esta situación, sin embargo, no es habitual, por lo que las densidades deberán ser aprendidas a partir de un conjunto de muestras de entrenamiento. Si suponemos que las densidades condicionales de clase se ajustan a una forma determinada por mencionar un ejemplo, una o varias gaussianas multivariadas pero algunos de sus parámetros son desconocidos ya sea las medias y/o matrices de covarianza, estaremos ante una aproximación paramétrica. Bajo este contexto, la solución al problema consiste en reemplazar los parámetros desconocidos por sus 
valores estimados. Por otra parte, si no se presupone una forma determinada de las densidades condicionales de clase, estaremos ante una aproximación no paramétrica. En este caso, debe estimarse la función de densidad o bien construir directamente las fronteras de decisión a partir de las muestras de entrenamiento. Otra vertiente que se presenta en el reconocimiento estadístico es la que se refiere a los modelos de aprendizaje supervisado y no supervisado. La diferencia entre estos es que en el caso del aprendizaje supervisado las muestras de entrenamiento están etiquetadas, conociendo con ello de antemano la clase a la que pertenecen, mientras que la etiqueta de clase e incluso el número de éstas es desconocida en el caso no supervisado.

La tabla 3.1 resume algunas aproximaciones al reconocimiento estadístico de patrones mencionadas en líneas anteriores. A medida que se va desplazando hacia abajo, la información disponible va disminuyendo y con ello, la dificultad en la clasificación aumenta.

Tabla 3.1: Diferentes aproximaciones al reconocimiento estadístico de patrones.

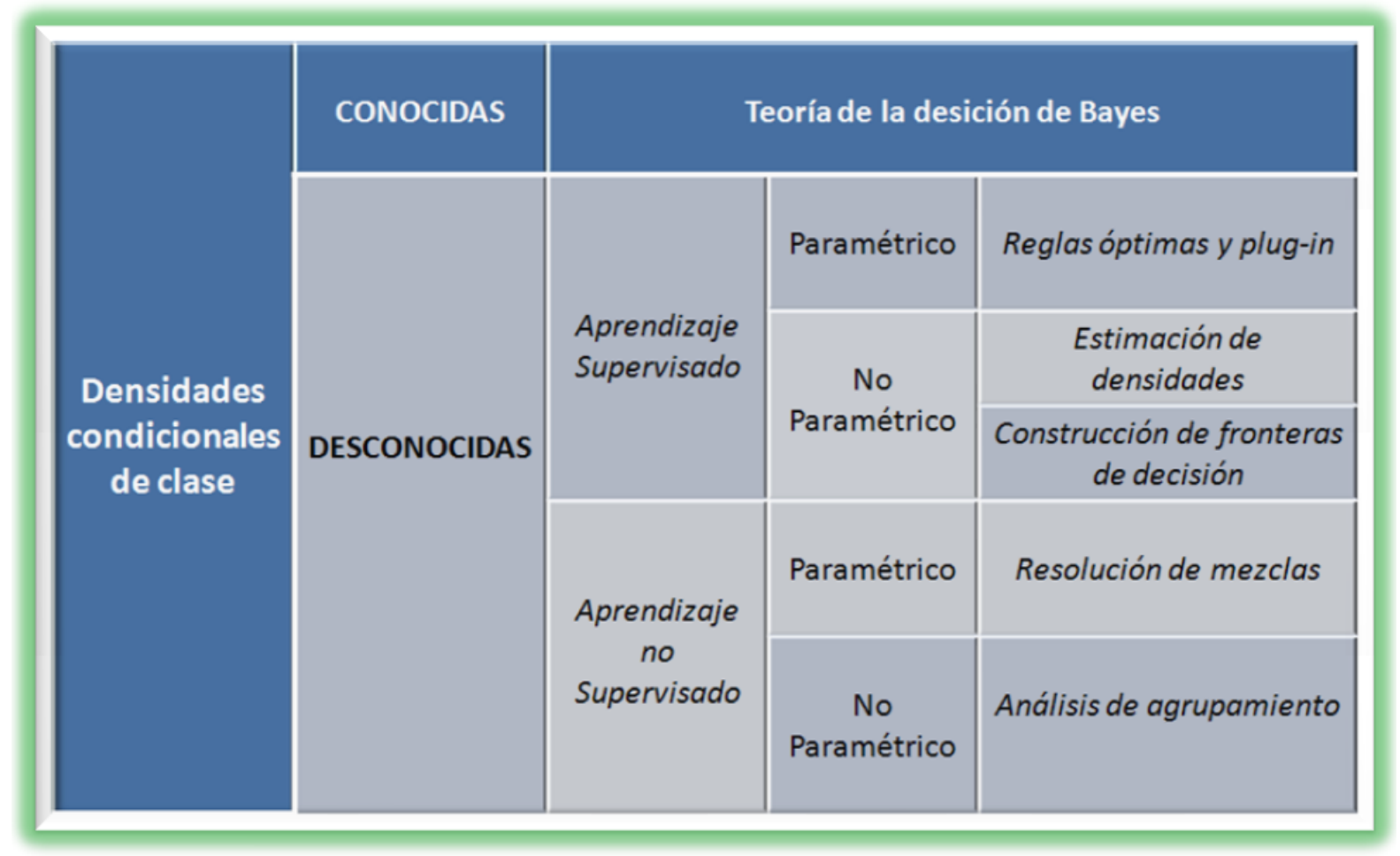

\subsection{Clasificador probabilístico: Regla de Bayes}

El proceso de toma de decisión en el reconocimiento de patrones estadístico se puede resumir de la forma siguiente: dado un patrón, éste ha de ser asignado en una de las $c$ categorías $\omega_{1}, \omega_{2}, \cdots, \omega_{c}$ basándose en el vector de $k$ valores de características $x=x_{1}, x_{2}, \cdots, x_{k}$. Se asume que las características tienen una densidad de probabilidad, conocida como densidad condicional de clase. De este modo, un vector $x$ de un patrón que pertenezca a la clase $\omega$ es visto como una observación o punto en el espacio $k$-dimensional escogido aleatoriamente de la función de probabilidad condicional de la clase $p\left(x \mid \omega_{i}\right)$.

La probabilidad de que una muestra cualquiera, de la cual no se tiene ningún tipo de informa- 
ción, pertenezca a la clase $\omega_{i}$ es lo que se conoce como probabilidad a priori $P\left(\omega_{i}\right)$. La información que aportan las características del objeto debería ocuparse para maximizar la fiabilidad de la clasificación, es decir, resultaría muy útil disponer de un mecanismo que nos proporcionara, a partir de estas observaciones, información sobre la probabilidad a posteriori $P\left(\omega_{i} \mid x\right)$. La manera de obtener esta probabilidad constituye la diferencia entre los distintos métodos de clasificación.

Sea $p\left(x \mid \omega_{i}\right)$ la función de densidad de probabilidad condicional de $x$ para una determinada clase $\omega_{i}$. Supongamos que conocemos las probabilidades a priori $P\left(\omega_{i}\right)$ y las densidades condicionales $p\left(x \mid \omega_{i}\right)$ entonces, la regla de Bayes permite calcular la probabilidad a posteriori de la siguiente manera

$$
P\left(\omega_{i} \mid x\right)=\frac{p\left(x \mid \omega_{i}\right) \cdot P\left(\omega_{i}\right)}{p(x)} \Leftrightarrow p(x)=\sum_{i=1}^{c} p\left(x \mid \omega_{i}\right) \cdot P\left(\omega_{i}\right)
$$

Una vez obtenida la probabilidad a posteriori de cada clase, se escogerá aquella clase que presente el máximo valor:

$$
P\left(\omega_{i} \mid x\right)>P\left(\omega_{j} \mid x\right), \quad 1 \leq i, j \leq c, \quad i \neq j \Rightarrow x \in \omega_{i}
$$

El criterio anterior constituye la regla de decisión de Bayes de mínimo error, en la cual se basan prácticamente, de algún manera, la mayoría de los métodos de clasificación estadísticos. Sin embargo en la mayoría de los caso, la probabilidad a posteriori no se podrá calcular de forma directa, ya que no se conocerá con certeza $p\left(x \mid \omega_{i}\right)$. Para ello será necesario estimar dichas probabilidades mediante algún proceso de entrenamiento a partir de un conjunto de muestras.

\subsection{Estimación paramétrica y no paramétrica}

Se puede distinguir entre los procedimientos que no hacen uso de ningún tipo de suposición respecto a la naturaleza de las funciones de densidad a estimar y aquellos que asumen que dichas densidades responden a una determinada distribución paramétrica. Los primeros se denominan métodos no paramétricos (MNP) y los últimos métodos paramétricos (MP).

En los MP el problema se reduce a estimar, a partir del conjunto de entrenamiento, los parámetros de la distribución, supuestamente conocida, que siguen las muestras de cada clase. En RP se utiliza con frecuencia dos métodos de estimación para los parámetros basados en distintos criterios: estimación por máxima verosimilitud y estimación bayesiana.

El primero de ellos ve los parámetros como cantidades con valores fijos pero desconocidos y la mejor estimación en este caso es la que maximiza la probabilidad de obtener las muestras observadas en el conjunto de entrenamiento. Los métodos bayesianos, en contraparte, ven los parámetros como variables aleatorias con una distribución a priori conocida donde la observación de las muestras de entrenamiento van modificando los valores de los parámetros iniciales.

En los MNP, la función de densidad de cada clase, la cual da una idea de la probabilidad que en un punto del espacio de representación aparezca una muestra de esta clase, se estima localmente llevando la cuenta de cuántos prototipos aparecen en cada región local del espacio. 


\subsection{Clasificadores basados en distancias}

Existen dos métodos ampliamente conocidos para la estimación no paramétrica de densidades mediante métodos de vecindad basados en distancias: las ventanas de Parzen y los $k$-vecinos más cercanos. A continuación se expone brevemente, la última de estas técnicas.

\subsubsection{El clasificador de los $k$ vecinos mas cercanos}

Sea $R$ una región del espacio de características $H$ (conjunto de entrenamiento) que contienen a $x$ y $V$ el área de esta región

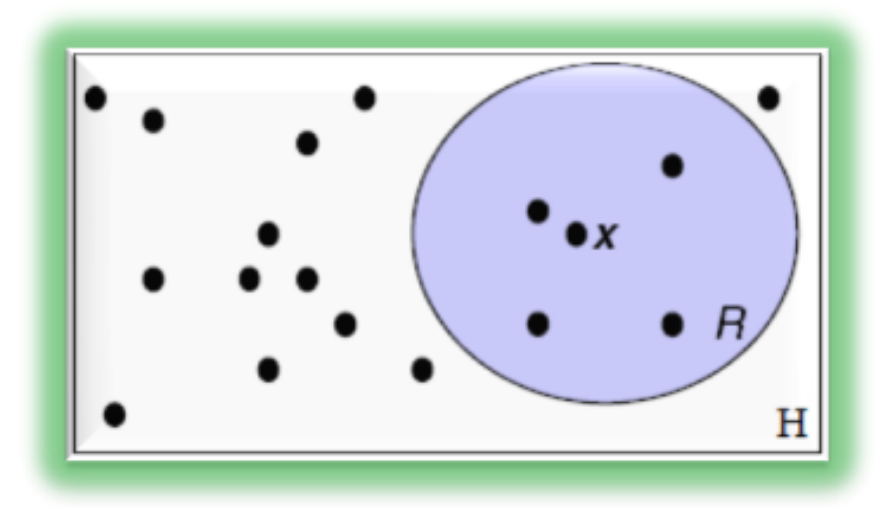

Figura 3.2:

Esquema de las consideraciones generales para la estimación de $p(x)$.

Desde una perspectiva general, la regla de los $k$-vecinos más cercanos fija el parámetro $k$ (número de muestras o prototipos contenidas en $R$ ) y obtiene el volumen $V$ de acuerdo al espacio ocupado por los $k$ prototipos más cercanos a $x$. De este modo, la región $R(x)$ alrededor de $x$ depende, para cada muestra, de la distribución del conjunto de prototipos, siendo menor en regiones de mayor densidad y mayor en regiones con menor número de prototipos. La función de núcleo o kernel asociada sería, en principio, uniforme, aunque en ocasiones se emplean funciones que dependen de la distancia. A continuación se hace un análisis formal de la regla de los $k$-vecinos más cercanos.

Para una función de densidad de probabilidad multivariada $p\left(x \mid \omega_{c}\right)$ de la clase $\omega_{c}$, la probabilidad $p$ de que un punto cualquiera $x_{o}$ caiga en la región local $R(x)$ es

$$
p=\int_{R} p\left(x_{o} \mid \omega_{c}\right) d x_{o}
$$

Si la región $R$ es pequeña y $V$ corresponde a su volumen entonces, se puede hacer la aproximación siguiente

$$
p \approx p\left(x \mid \omega_{c}\right) V \Rightarrow p\left(x \mid \omega_{c}\right) \approx \frac{p}{V}
$$

Donde $\frac{p}{V}$ será una aproximación suavizada de $p\left(x \mid \omega_{c}\right)$, es decir, el valor medio de $p\left(x_{o} \mid \omega_{c}\right)$ en la región local $\mathrm{R}$ alrededor de $x$. Si $R$ es suficientemente pequeña, entonces $p\left(x_{o} \mid \omega_{c}\right)$ no se desviará mucho de $p\left(x \mid \omega_{c}\right)$. De este modo, si se estima $p$ se puede estimar directamente $p\left(x \mid \omega_{c}\right)$. Por tanto $p$ puede estimarse simplemente viendo qué proporción de los $n_{c}$ prototipos 
de la muestra caen dentro de $R$. Si $k$ es el número de puntos de la muestra de la clase $w_{c}$ que caen en $R$, entonces la función de densidad de probabilidad en $R$ se estima

$$
\hat{p}=\frac{k}{n_{c}} \Rightarrow \hat{p}\left(x \mid \omega_{c}\right)=\frac{k}{n_{c} \cdot V}
$$

Ahora bien, para establecer $V$ en función de los datos y garantizar que sea variable según la zona del espacio en la que se defina, se fija $k$ y posteriormente, se determina el volumen $V$ necesario para abarcar los $k$ puntos de la clase más cercanos a $x$. Si la densidad es alta, $V$ será relativamente pequeño, mientras que en zonas de menor densidad, el volumen crecerá. El uso de este estimador se conoce como método volumétrico de los $k$-vecinos más cercanos.

Si se tiene en cuenta que en la práctica lo que se pretende es utilizar el estimador como un clasificador capaz de discriminar entre clases para nuevas observaciones, se puede extender el método de la siguiente manera: dado un punto $x$ y una combinación de $n$ prototipos pertenecientes a distintas clases $\left(\sum_{c} n_{c}=n\right)$, se puede definir la hiperesfera de volumen $\mathrm{V}$ que abarque exactamente los $k$ puntos más próximos a $x$. Si se supone que entre estos $k$ puntos hay $k_{c}$ de la clase $w_{c}$, entonces se puede definir un estimador para la función de densidad para la clase $w_{c}$ de la forma

$$
\hat{p}\left(x \mid \omega_{c}\right)=\frac{k_{c}}{n_{c} \cdot V}
$$

Por otro lado los estimadores para la probabilidad a priori de la clase $\hat{P}\left(\omega_{c}\right)$ y la probabilidad $\hat{p}(x)$ son

$$
\hat{P}\left(\omega_{c}\right)=\frac{n_{c}}{n} \quad \hat{p}(x)=\frac{k}{n \cdot V}
$$

A partir de estas probabilidades, y según la regla de decisión de Bayes, se puede estimar la probabilidad a posteriori como

$$
\hat{p}\left(\omega_{c} \mid x\right)=\frac{\hat{p}\left(x \mid \omega_{c}\right) \cdot \hat{P}\left(\omega_{c}\right)}{\hat{p}(x)}=\frac{\frac{k_{c}}{n_{c} \cdot V} \cdot \frac{n_{c}}{n}}{\frac{k}{n \cdot V}}=\frac{k_{c}}{k}
$$

lo anterior nos lleva a la regla de clasificación denominada votación entre los $k$-vecinos más cercanos $(k-N N)$

$$
x \in \omega_{i} \quad \text { si } \quad k_{i}=\operatorname{máx}_{c}\left(k_{c}\right)
$$

esto es, se clasifica $x$ como perteneciente a la clase más votada entre los $k$ vecinos. En resumen, en el método de votación se obtienen los $k$ vecinos más cercanos a $x$ de entre todas las muestras y se cuentan el número de vecinos pertenecientes a cada clase. La clase mayoritaria será la que se asigne a $x$, sin necesidad de aplicar explícitamente la regla de Bayes. En caso de empate se pueden aplicar diversas estrategias. Quizás la más rigurosa sea ir reduciendo $k$ en una unidad hasta deshacer el empate. Otra posibilidad de menor coste consiste en asignar la clase del vecino más cercano. 


\subsection{Segmentación/Clasificación mediante análisis cluster}

El análisis cluster $\boldsymbol{A} \boldsymbol{C}$ o de grupos [14] es un método estadístico multivariado de clasificación automática de datos cuyo objetivo principal consiste en determinar la existencia o no de grupos similares de variables que tiendan a agruparse en conglomerados, es decir, dada una muestra de individuos (de cada uno de los cuales disponemos de un conjunto de observaciones), el AC los clasificará en grupos lo más homogéneos posible siempre en base al conjunto de variables observadas y los individuos que resulten clasificados dentro de un mismo grupo serán tan similares como sea posible. Este proceso se genera a partir de una tabla de casos-variables donde se intenta situar a los individuos o casos en grupos, conglomerados o clusters homogéneos no conocidos de antemano pero sugeridos por la estructura presente en los datos, de manera tal que individuos considerados como similares sean asignados a un mismo cluster mientras que los individuos diferentes se localicen en clusters distintos.

Existen dos grandes grupos de AC: aquellos que asignan a los individuos a grupos diferenciados que el propio análisis configura, sin que unos dependan de los otros, son conocidos como no jerárquicos, y aquellos que configuran grupos con estructura arborescente, de forma que clusters de niveles inferiores se engloban en los de niveles superiores, se denominan jerárquicos. El primero de los métodos puede a su vez producir clusters disjuntos, aquellos donde cada individuo pertenece a uno y solo uno de los clusters, o bien traslapados donde un individuo puede pertenecer a más de un grupo.

Antes de iniciar el AC deben tomarse tres desiciones fundamentales:

1. Seleccionar las variables relevantes para la identificación de los posibles grupos existentes

2. Definición de la medida o métrica de proximidad entre los individuos a ser utilizada básicamente, similitudes y distancias ya que los conglomerados que se forman lo hacen en base a las proximidades entre variables o individuos

3. Y la elección del criterio con el cual se agruparan a los individuos en los conglomerados

\subsubsection{Métricas de distancia y similitud}

La proximiad expresa la semejanza existente entre individuos o variables, es decir, corresponde al grado de asociación que existe entre ellos. Ésta a su vez puede medir ya sea distancia o bien similaridad entre conjuntos de individuos. El valor obtenido por una medida de distancias es tanto mayor cuanto más alejados están los individuos entre los que se realiza la medición y por el contrario, el valor de similitud que se obtiene es tanto mayor cuanto más próximos están los individuos considerados.

Un primer ejemplo de distancia corresponde a la distancia euclidiana definida por (3.10)

$$
d(A, B)=\sqrt{\sum_{i}\left(A_{i}-B_{i}\right)^{2}}
$$

Un segundo ejemplo es la distancia de Mahalanobis $D^{2}$, que corresponde a la distancia al cuadrado entre los centroides de dos poblaciones. Recordemos que el centroide de una población 
es el centro de gravedad de esta población en base a un conjunto de variables como el vector de medias de estas, teniendo en mente que el centroide de una población es el sinónimo de la media para el caso multivariado. La $D^{2}$ permite situar poblaciones en un espacio de $r$ dimensiones, siendo $r$ el número de variables consideradas en el estudio.

De manera formal, sean $p$ poblaciones de $n_{1}, n_{2}, \cdots, n_{p}$ individuos cada una y para cada población se conocen $r$ variables $x_{1}, x_{2}, \cdots, x_{r}$ de modo que a cada población $k$ le corresponde una matriz de observaciones de orden $n_{k} x r$. Se dispone por tanto de $p$ matrices del mismo orden con $k=1, \cdots, p$. A partir de estos datos, Mahalanobis define la distancia entre los centroides de los grupos $p$ y $q$ como en 3.11

$$
D_{p q}^{2}=\left(\mu_{p}-\mu_{q}\right)^{\prime} \cdot \sum^{-1} \cdot\left(\mu_{p}-\mu_{q}\right)
$$

Donde $\mu_{p}$ y $\mu_{q}$ son vectores columna que contienen las medias de las variables de los grupos respectivos y $\sum$ es la matriz de varianzas covarianzas intragrupos de los grupos conjuntamente.

Otros tres ejemplos de distancia entre dos variables $x$ e $y$ son:

1. La distancia de Manhattan que se define como en 3.12

$$
B(x, y)=\sum_{i}\left|x_{i}-y_{i}\right|
$$

2. La distancia de Minkowski que se define como en 3.13

$$
M(x, y)=\sum_{i}\left(\left|x_{i}-y_{i}\right|^{p}\right)^{\frac{1}{p}}
$$

3. La distancia de Chebychev que se define como en (3.14)

$$
C(x, y)=\operatorname{Max}\left|x_{i}-y_{i}\right|
$$

Entre las medidas de similitud se tienen por mencionar algunos, los coeficientes de correlación de Pearson y Sperman, la información mutua, los múltiples coeficientes de asociación entre variables, $\lambda, \tau$, etc

\subsubsection{Clusters no jerárquicos}

Este análisis requiere que el investigador fije de antemano el número de clusters en que quiere agrupar sus datos, como puede no existir un número definido de grupos o generalmente éste no se conoce, la prueba deberá ser repetida variando este número con el fin de tantear la clasificaión que mejor se ajuste al objetivo del problema o bien la de más clara interpretación.

Estos métodos buscan realizar una sóla partición de los individuos en $K$ grupos asignándolos a éstos, mediante algún proceso que optimice el criterio de selección. 


\section{El algoritmo de las $k$-medias}

El algoritmo de las $k$-medias [15] constituye un método no jerárquico de reasignación, ya que permite que un individuo asignado a un grupo en un determinado paso del proceso sea reasignado a otro grupo distinto en un paso posterior sólo si esto optimiza el criterio de selección. Este algoritmo es uno de los más importantes desde el punto de vista conceptual y práctico, el cual parte de unas medias arbitrarias y, mediante pruebas sucesivas, contrasta el efecto que sobre la varianza residual tiene la asignación de cada uno de los individuos a cada uno de los grupos. El valor mínimo de varianza determina una configuración de nuevos grupos con sus respectivas medias. Se asignan otra vez todos los individuos a estos nuevos centroides en un proceso que se repite hasta que ninguna transferencia pueda disminuir la varianza residual; o se alcance otro criterio de parada como un número limitado de iteraciones o simplemente que la diferencia obtenida entre los centroides de dos pasos consecutivos sea menor que un valor prefijado.

El procedimiento configura los grupos maximizando, a su vez, la distancia entre sus centros de gravedad. Como la varianza total es fija, minimizar la residual hace máxima la factorial o intergrupos y puesto que minimizar ésta es equivalente a conseguir que sea mínima la suma de distancias al cuadrado desde los individuos a la media del cluster al que van a ser asignados, es esta distancia euclidiana al cuadrado la utilizada por el método y la mostrada en el argumento de la función objetivo a minimizar definida por la relación en (3.15)

$$
J=\sum_{j=1}^{k} \sum_{i=1}^{n}\left\|x_{i}^{(j)}-c_{j}\right\|^{2}
$$

Como cualquier otro método de clasificación no jerárquica, proporciona una solución final única para el número de clusters elegido la selección más apropiada al fenómeno de tal número deberá basarse tanto en criterios matemáticos como de interpretabilidad.

A continuación en la figura 3.3, se presenta un diagrama descriptivo de los pasos que constituyen al algoritmo de las $k$-medias:

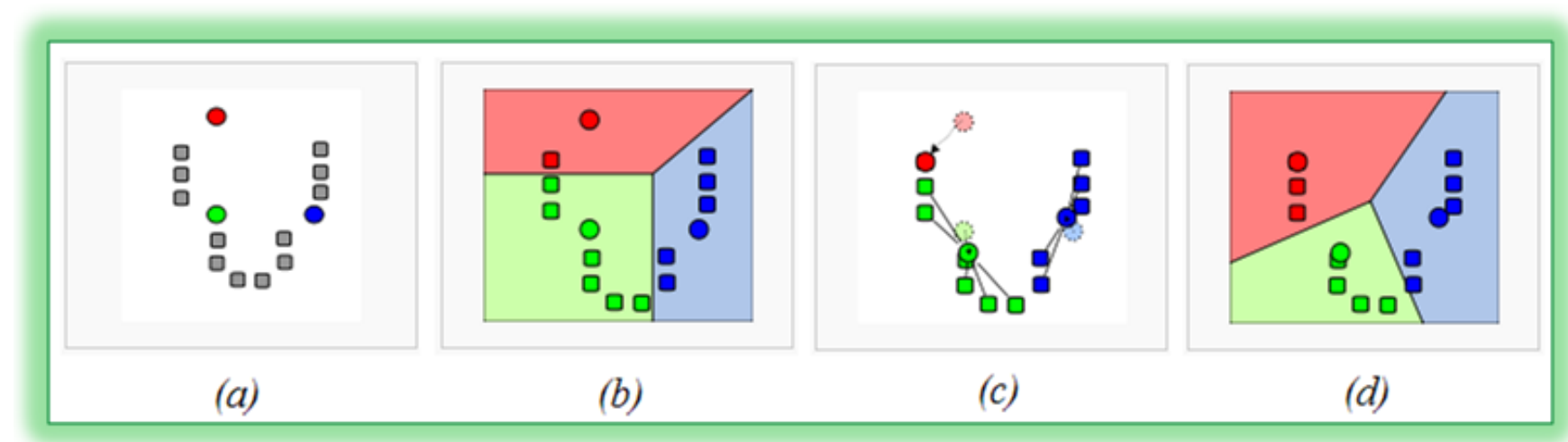

Figura 3.3:

Esquema representativo del proceso efectuado por el algoritmo convencional $k$-medias (a) $k$ medias iniciales (en este caso tres), son aleatoriamente seleccionadas a partir del conjunto de datos (b) $k$ grupos son formados por la asociación de cada observación más próxima a la media actual (circulos en color)

(c) Los centroides de cada uno de los $k$ grupos se transforman en las nuevas medias

(d) Los pasos (b) y (c) son repetidos hasta que la convergencia ha sido alcanzada. 


\title{
Capítulo 4
}

\section{Los Mapas Auto-Organizados (SOM)}

\author{
Los conceptos y principios fundamentales de la ciencia, \\ son invenciones libres del espíritu humano.
}

ALBERT EINSTEIN

En 1982, Teuvo Kohonen presentó un modelo de red neuronal artificial denominada mapas Auto-Organizados SOM, por sus siglas en inglés Self-Organizing Maps, basado en evidencias descubiertas a nivel cerebral observando que en el corteza cerebral de los animales superiores aparecen zonas donde las neuronas detectoras de rasgos similares se encuentran topológicamente ordenadas; de forma que las informaciones captadas del entorno a través de los órganos sensoriales, se representan internamente en forma de mapas bidimensionales. De esta forma, como se muestra en la figura 4.1, existe una zona dedicada al tratamiento de la visión, otra diferente para el oído, otra para las funciones motoras, etc.

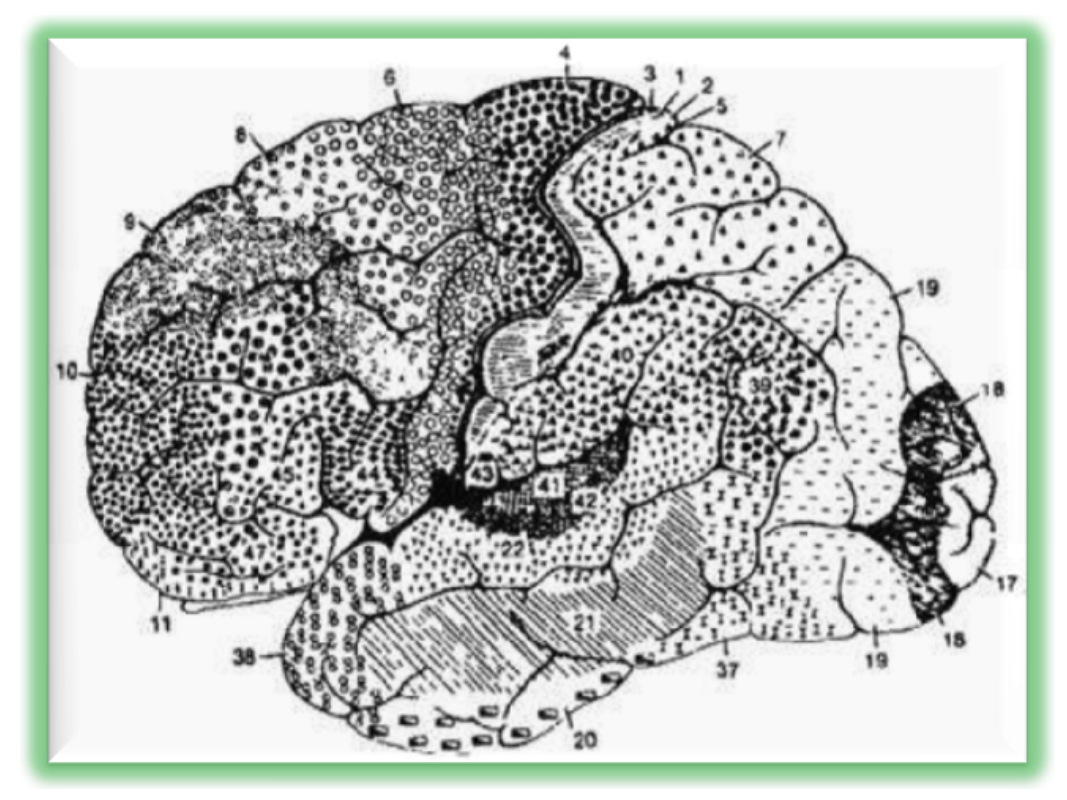

Figura 4.1:

Distinción de áreas: motoras $(4,6,8)$, visuales $(17,18,19)$ y auditivas $(41,42)$ situadas de forma ordenada en la corteza cerebral.

Aunque en gran medida esta organización neuronal está predeterminada genéticamente, es probable que parte de ella se origine mediante el aprendizaje. Esto sugiere, por tanto, que el cerebro podría poseer la capacidad inherente de formar mapas topológicos de las informaciones 
recibidas del exterior. Por otra parte, también se ha observado que la influencia que una neurona ejerce sobre las demás es función de la distancia entre ellas, siendo muy pequeña cuando están muy alejadas y muy grande cuando están muy cerca la una de la otra [16]. Basándose en estas ideas se desarrollaron los mapas auto-organizados.

\subsection{Introducción al mapa auto-organizado}

El SOM se asocia a un aprendizaje no supervisado ya que al no disponer de ninguna salida objetivo hacia la cual la red debiera tender, no existe ninguna referencia externa que indique si la red neuronal está operando correcta o incorrectamente [16].

Por ello, el objetivo principal del SOM, es descubrir rasgos comunes, regularidades o bien categorías en el conjunto de datos de entrada e incorporarlos a su propia estructura interna de conexiones, es decir, las neuronas que constituyen al mapa deberán auto-organizarse en función siempre de los estímulos procedentes del exterior llevando así un proceso de categorización de los datos de entrada. Las clases o categorías serán creadas por la misma red neuronal durante el transcurso de su aprendizaje, puesto que este proceso es no supervisado, mediante las correlaciones existentes entre los datos.

De esta manera, lo que se pretende, es que al presentarle a la red neuronal algún patrón de entrada, sólo una de las neuronas de salida se active, así, cada una de estas neuronas competirán por activarse quedando al final del entrenamiento algunas neuronas vencedoras y otras anuladas haciéndolas tender a sus valores de respuesta mínimos.

Básicamente los SOMs pueden cumplir con dos funcionalidades:

1. Actuar como clasificadores, encontrando patrones en los datos de entrada y clasificándolos en grupos según estos patrones.

2. Representar datos multidimensionales en espacios de mucha menor dimensión, normalmente una o dos dimensiones, preservando la topología de la entrada. Esto es muy útil cuando se trabaja con espacios multidimensionales que el ser humano no es capaz de representar.

\subsection{Arquitectura del mapa auto-organizado}

La arquitectura de una red neuronal artificial determina qué posibles interconexiones neuronales son factibles entre las neuronas de la red. La red SOM se compone de dos capas una de neuronas y otra de datos observados [17] que fueron obtenidos de los registros de EEG.

\section{La capa de entrada}

Se encarga de recibir y transmitir a la capa de salida la información procedente del exterior y se compone de $N$ observaciones, una por cada variable de entrada.

\section{La capa de salida}

Formada por $M$ neuronas encargadas de procesar la información de la capa de entrada y formar el mapa de rasgos. 
La conexión entre estas dos capas siempre es en dirección de los datos de entrada $X=$ $\left\{x_{1}, x_{2}, \cdots, x_{N}\right\}$ hacia cada uno de los nodos o neuronas del arreglo bidimensional que defina a la capa de salida. La geometría de esta última capa puede ser definida de manera regular ya sea formando una malla rectangular o hexagonal o bien, de manera irregular aunque para efectos visuales, conviene más definir una malla regular tal y como puede observarse en la figura 4.2

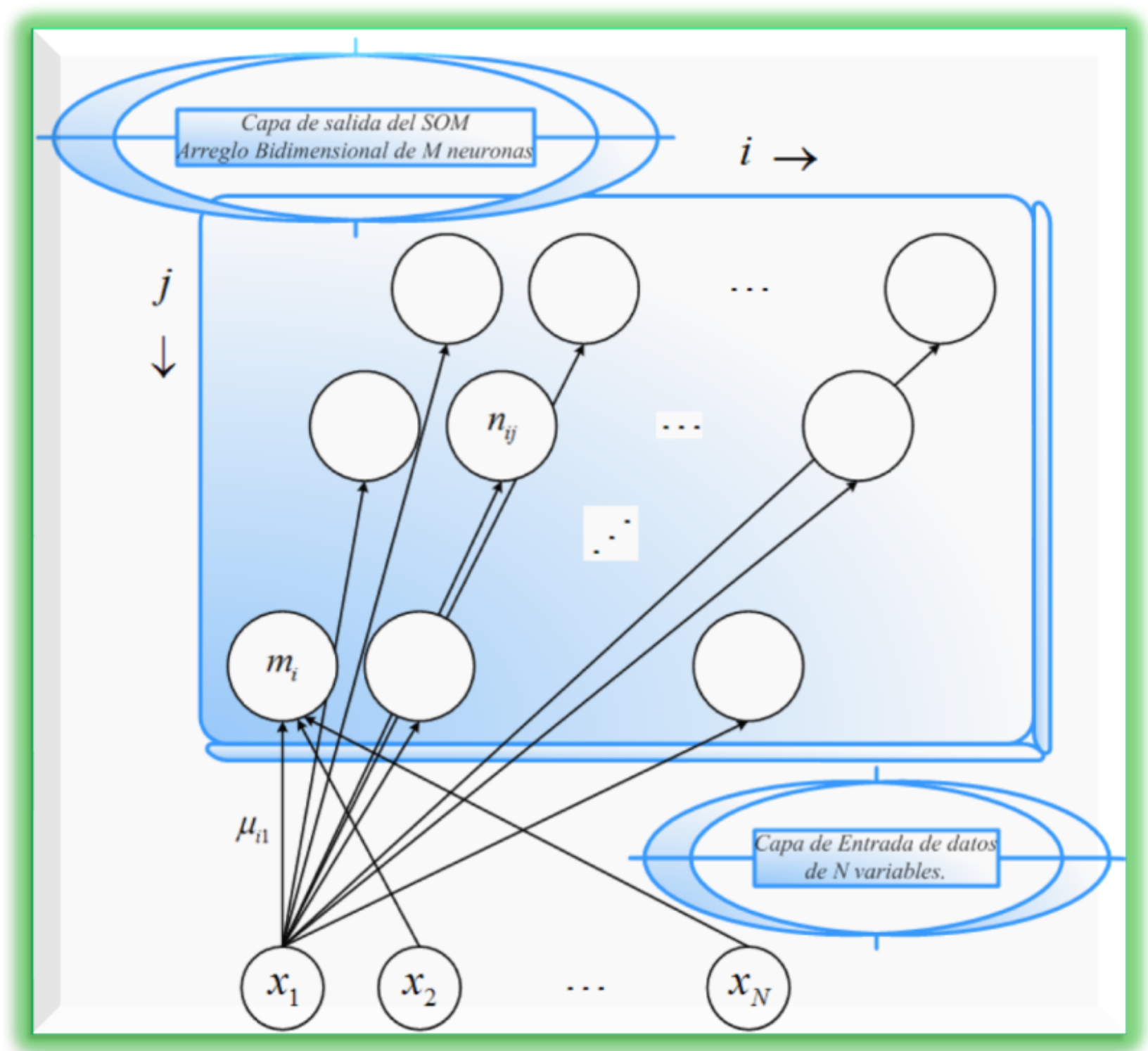

Figura 4.2:

Diagrama descriptivo de la arquitectura y funcionalidad de los Mapas Auto-Organizados.

Cada variable de entrada $x_{n}$, se conecta con cada una de las neuronas de salida $n_{i j}$ mediante un peso $\mu_{i j}$, por lo que cada una de estas neuronas tiene asociado un vector paramétrico de pesos $\omega_{i}$ y el conjunto de todos estos vectores $\omega_{i}$ forman al codebook del mapa.

$$
\omega_{i}=\left\{\mu_{i 1}, \mu_{i 2}, \mu_{i 3}, \cdots, \mu_{i N}\right\} \in \mathbb{R}^{N}
$$

De esta manera, podría decirse que el algoritmo SOM define una proyección o cartografía de una entrada de datos que pertenecen a un espacio $\mathbb{R}^{N}$ de alta dimensionalidad sobre un arreglo o mapa bidimensional de nodos conocidos como neuronas. 


\subsubsection{Función de vecindad}

Entre todas las neuronas del arreglo 2D existen conexiones laterales de excitación e inhibición implicitas ya que aunque físicamente no se encuentren unidas, cada una tendrá cierta influencia sobre sus vecinas, lo cual se logra por la aplicación de una función denominada función de vecindad que produce la topología o estructura del mapa definida por 4.2

$$
h_{i j}(t)=h\left(r_{i}, r_{j} ; t\right)
$$

Donde $r_{i}$ y $r_{j}$ son las localizaciones geométricas en la red neuronal de los nodos $i$ y $j$ respectivamente y $t$ es la coordenada con el tiempo. El grado de interacción es frecuentemente variable del tiempo y las neuronas adyacentes a la neurona $j$ pertenecen a una vecindad $N_{j}$.

Las funciones de vecindad más utilizadas son las mostradas en 4.3 aunque normalmente ésta suele seguir una curva Gaussiana centrada en la posición de la neurona ganadora. En la figura 4.3 se muestran algunas posibles morfologías para los kernels de vecindad.

$$
h_{i c}(t)=\left\{\begin{array}{cc}
u\left(\sigma_{t}-d_{c i}\right) & (a) \\
e^{\frac{-d_{c i}^{2}}{2 \sigma_{t}^{2}}} & (b) \\
e^{\frac{-d_{c i}^{2}}{2 \sigma_{t}^{2}}} u\left(\sigma_{t}-d_{c i}\right) & (c) \\
\operatorname{máx}\left\{0,1-\left(\sigma_{t}-d_{c i}\right)^{2}\right\} & (d)
\end{array}\right.
$$

Donde $\sigma_{t}$ corresponde al radio de vecindad al tiempo $t, d_{c i}=\left\|r_{c}-r_{i}\right\|$ es la distancia entre las unidades $c$ e $i$ del mapa y $u(t)$ es la función escalón: $u(t)=0$ si $t<0$ y $u(t)=1$ si $t>0$.

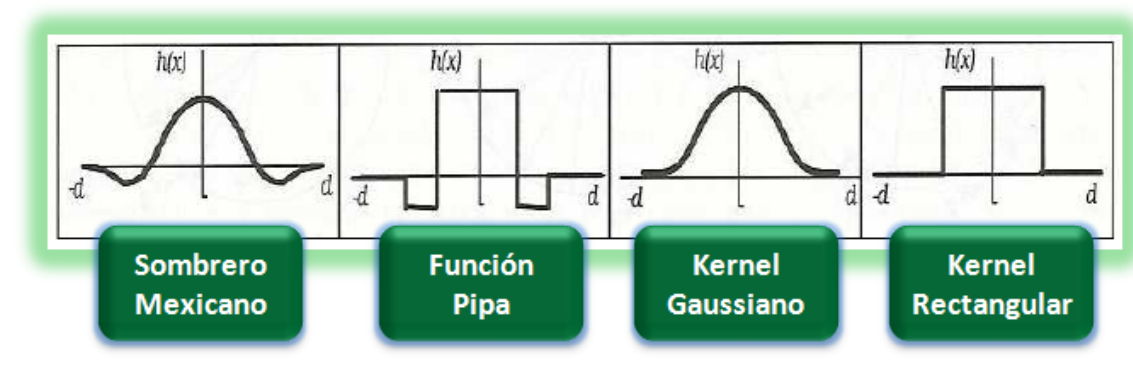

Figura 4.3:

Ejemplos gráficos de algunos tipos de función de vecindad.

La topología y el número de neuronas permanece fijo desde el comienzo del entrenamiento, la cantidad de estas neuronas determinará la suavidad de la proyección lo que influye directamente en el ajuste y capacidad de generalización del SOM.

A lo largo del proceso de aprendizaje $h(t)$ puede ir modificándose, al principio ésta podrá trabajar por ejemplo con una gaussiana aplanada que permita alimentar a muchas neuronas cercanas a la ganadora (vecindario grande) y progresivamente se irá haciendo más picuda (vecindario pequeño) alimentando sólo a las neuronas más cercanas a la ganadora, terminando por influir sólo en la neurona ganadora. Este estrechamiento de $h(t)$ se consigue modificando su varianza, partiendo de una varianza alta y finalizando con una varianza baja. 
Sí llamamos radio de vecindad $R(t)$ a la apertura de $h(t)$ entonces, a lo largo del proceso ésta puede variar como en 4.4

$$
R(t)=R_{o}+\left(R_{f}-R_{o}\right) \cdot \frac{t}{t_{R}}
$$

Donde $R_{o}$ representa el radio inicial y tiene un valor grande mientras que $R_{f}$ es el radio final y tiene un valor igual a $1, t$ representa la iteración y $t_{R}$ el total de éstas.

\subsection{El Algoritmo de aprendizaje del mapa auto-organizado}

A continuación se muestra a meanera de esquema, cada uno de los pasos que conforman al algorítmo de aprendizaje de los mapas auto-organizados:

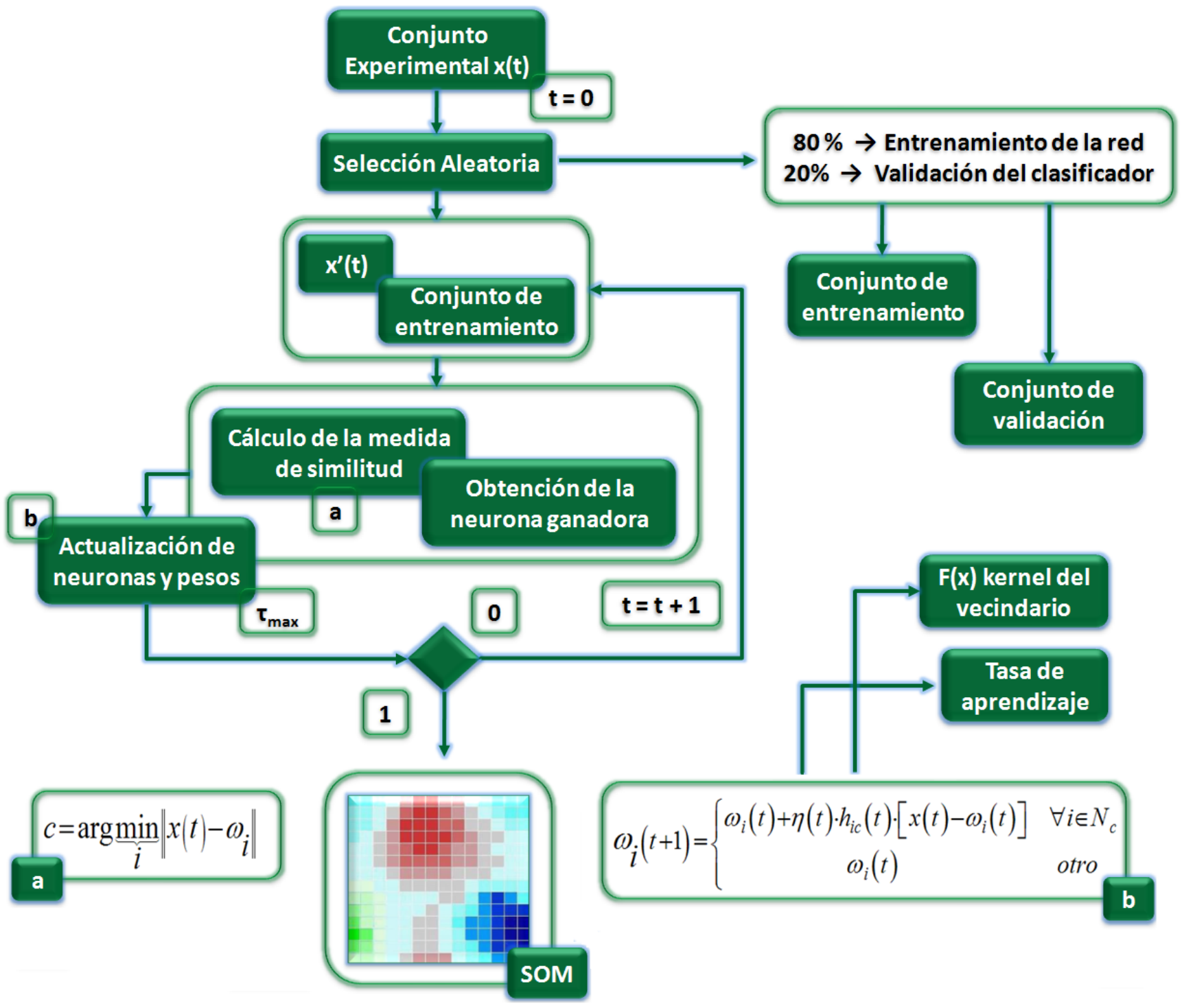

Figura 4.4:

Diagrama a bloques del funcionamiento del algoritmo de aprendizaje SOM. 
El proceso de aprendizaje del SOM es descrito de manera estructurada como:

1. Un vector $x_{i}$ es seleccionado de manera aleatoria del conjunto de datos de entrenamiento y es comparado con todos los vectores $\omega_{i}$ del codebook respecto a alguna métrica, usando por ejemplo la distancia euclidiana mostrada en 4.5 como medida de similitud

$$
\left\|x-\omega_{c}\right\|=\operatorname{Min}_{i}\left\{\left\|x(t)-\omega_{i}\right\|\right\}
$$

De esta manera, la más pequeña de las distancias euclidianas define al vector más próximo del codebook (BMU por sus siglas en inglés, Best Matching Unit/Nodo mejor relacionado).

2. Al encontrar el vector más proximo, el resto de los vectores del codebook son actualizados. El BMU junto con sus vecinos topológicos se mueven acercándose al vector $x$ en el espacio de datos.

La magnitud de esta atracción se rige por la tasa de aprendizaje $\eta(t)$ y la función de vecin$\operatorname{dad} h(t)$.

La tasa de aprendizaje $\eta(t)$, también varía a lo largo del proceso partiendo de un valor alto (con el que la modificación de los pesos es mayor) y disminuyendo lineal o exponencialmente (como se muestra en 4.6) hacia un valor bajo consiguiendo a lo largo del proceso de aprendizaje, modificar de menor manera en cada iteración los pesos correspondientes.

$$
\eta(t)=\eta_{o}+\left(\eta_{f}-\eta_{o}\right) \cdot \frac{t}{t_{\eta}} \quad \eta(t)=\eta_{o} \cdot\left(\frac{\eta_{f}}{\eta_{o}}\right)^{\frac{t}{t_{\eta}}}
$$

Mientras se produce la actualización de los $\omega_{i}$ y nuevos vectores se asignan al mapa, la $\eta(t)$ decrece gradualmente hacia cero, pasándole exactamente lo mismo al radio de vecindad.

Los valores de $\omega_{i}$ son adaptiva y gradualmente actualizados por el siguiente proceso de aprendizaje auto-organizado:

$$
\omega_{i}(t+1)=\left\{\begin{array}{c}
\omega_{i}(t)+\eta(t) h_{i c}(t)\left[x(t)-\omega_{i}(t)\right] \quad \forall i \in N_{c}(t) \\
\omega_{i}(t) \quad \forall i \notin N_{c}(t)
\end{array}\right.
$$

Donde $t$ es una variable entera o coordenada de tiempo discreto y $c$ corresponde al índice del vector paramétrico $\omega_{i}$ que es definido por $c=c\left(x ; \omega_{1}, \omega_{2}, \cdots, \omega_{N}\right)$

Los pasos 1) y 2) son repetidos hasta finalizar el entrenamiento y el número de iteraciones se fija de forma apriori para poder calcular las tasas de convergencia y aprendizaje. 


\subsubsection{Mapas auto-organizados como clasificadores}

Ahora bien, si permitimos que $X \in \mathbb{R}^{N}$ sea un vector de datos estocástico con función de densidad de probabilidad $p(X)$ entonces, durante el proceso de entrenamiento el SOM logrará formar una red elástica que se pliegue dentro de la nube de datos originales y así el algoritmo controlará a la red de modo tal que tienda a aproximar la densidad de los datos $p(X)$ haciendo que los vectores del codebook se acerquen a las áreas donde $p(X)$ es alta y eventualmente unos pocos se centrarán en áreas donde existe baja densidad.

El proceso anterior se ejemplifica por medio de la simulación mostrada en la figura 4.5

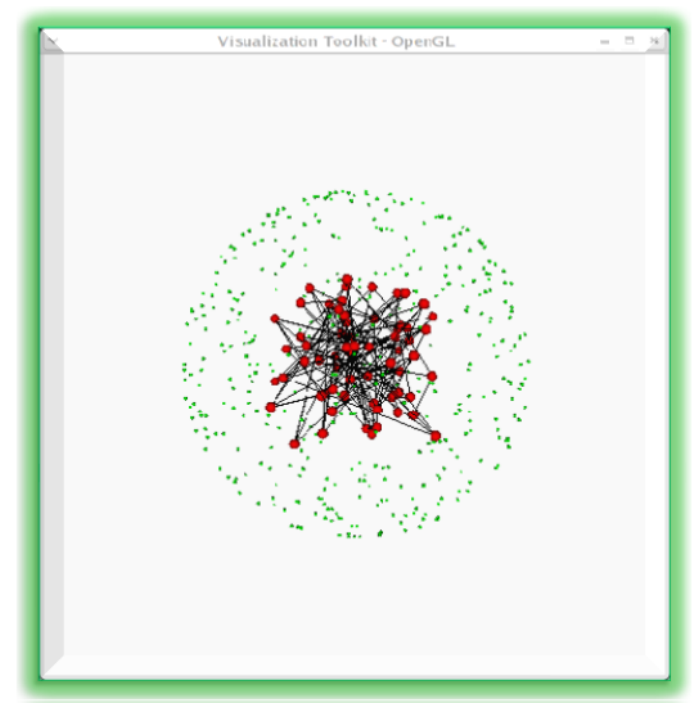

(a)

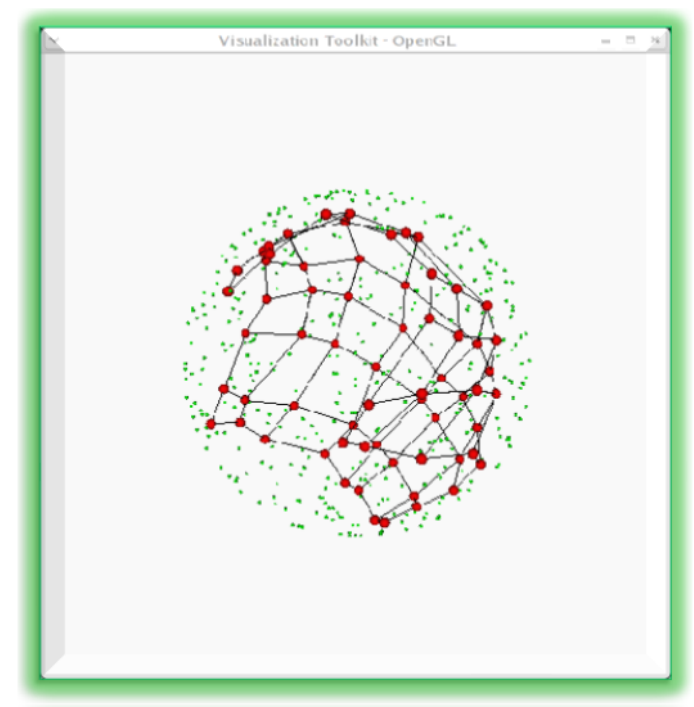

(c)

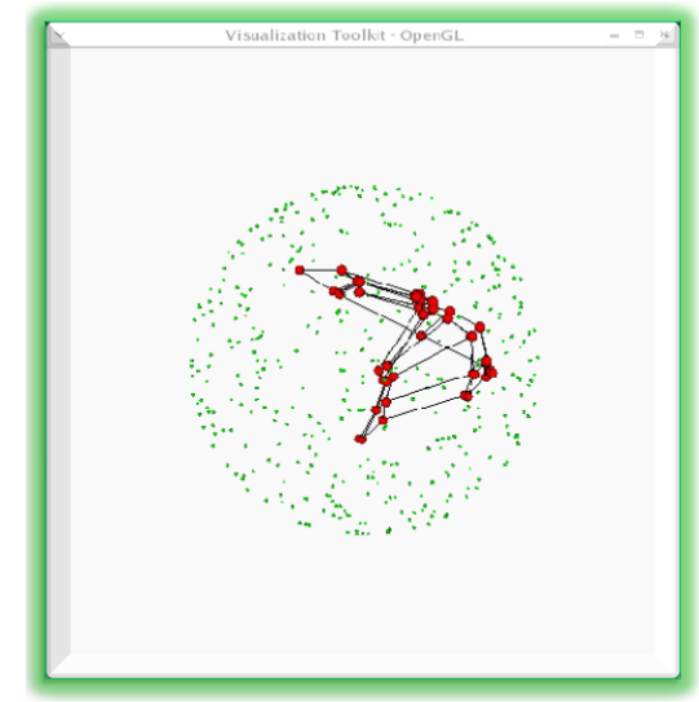

(b)

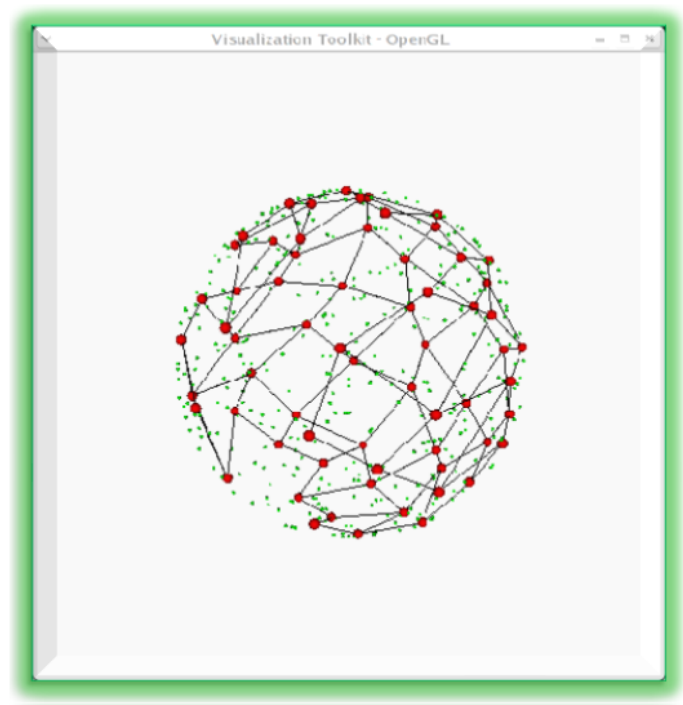

(d)

Figura 4.5:

Simulación del proceso de entrenamiento con datos uniformamente distribuidos sobre una esfera unitaria (a) Inicio del proceso de entrenamiento: iteración cero

$(b-d)$ Evolución en la iteración del proceso: comportamiento de la red elástica sobre el espacio de datos 


\section{Parte III}

Metodología 


\section{Capítulo 5}

\section{Bosquejo general}

Un sutil pensamiento erróneo puede dar lugar... a una indagación fructífera que revele verdades de gran valor.

ISAAC ASIMOV

En la figura 5.1 se presenta de forma esquemática cada uno de los bloques que conforman a la metodología seguida durante la realización de este trabajo.

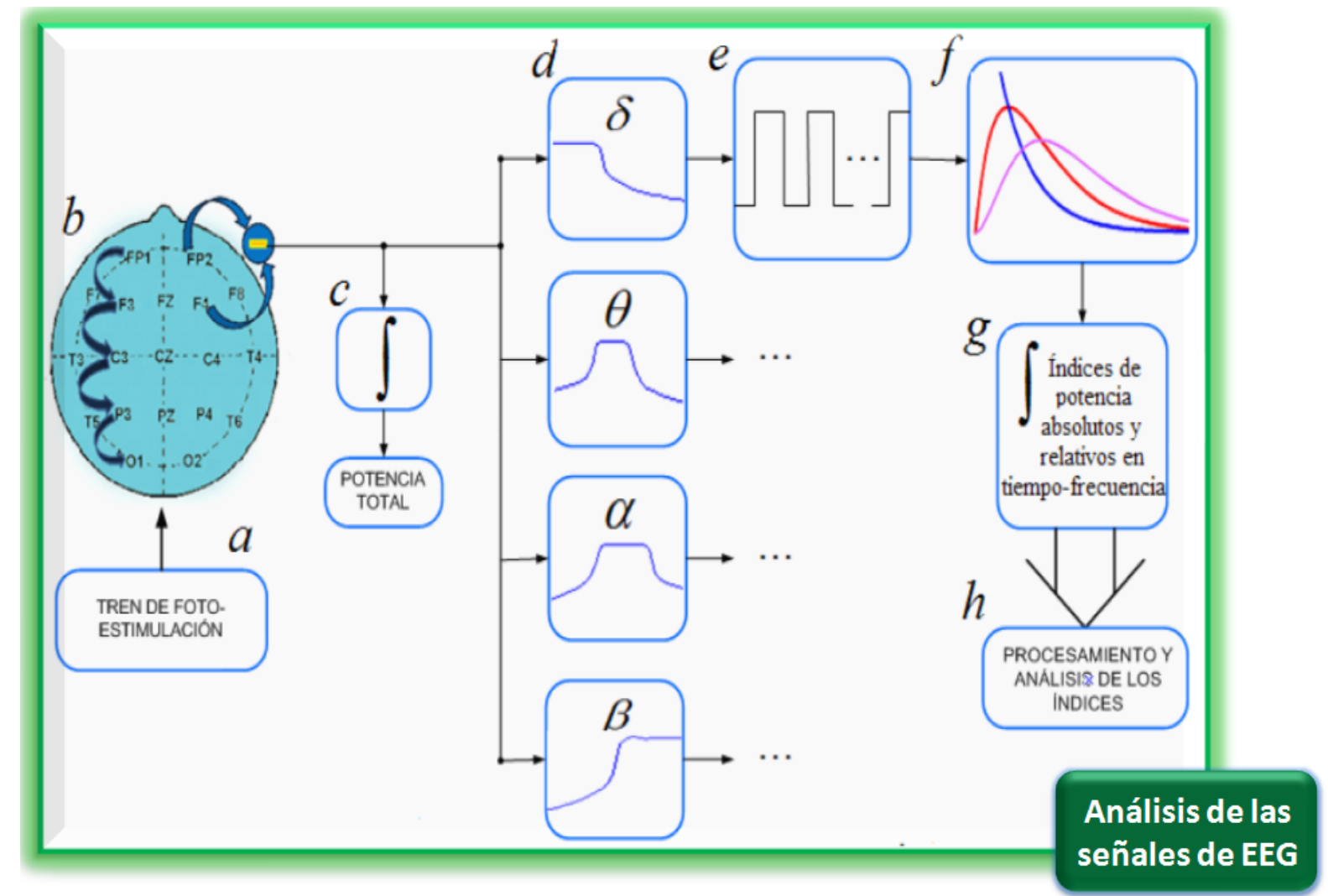

Figura 5.1: Representación esquemática de cada una de las etapas del procesamiento de las señales electroencefalográficas

a) Estimulación: Tren de veinte pulsos con diez foto-estimulaciones cada uno.

b) Adquisición: Obtención de 10 registros de EEG y posterior sustracción digital de cada par por hemisferio cerebral

c) Referencia de Potencia: Estimación del índice de potencia total para cada una de las señales diferencia a estudiar.

d) Filtrado: Separación de cada ritmo cerebral de las señales diferencia por la aplicación de un banco de filtros digitales.

e) Segmentación: Partición de las señales bipolares en ventanas referentes a la repetición y a la ubicación del estímulo.

f) Densidades Espectrales de Potencia DEP: Estimación de las DEP de cada uno de los ritmos por el método de Welch.

g) Extracción de características por sujeto: Cálculo de índices de potencia para rasgos frecuenciales y de energía para rasgos wavelet.

h) Procesamiento y Análisis de los índices: Aplicación de los Mapas Auto-Organizados para el análisis de los índices y registros. 
Se realizó un análisis de clasificación multivariada con rasgos provenientes de los registros electroencefalográficos de una población de 28 niños (referidos para evaluación psicológica por parte de su escuela) con edades entre los siete y doce años, de los cuales 18 fueron diagnosticados con TDAH (15 hombres y tres mujeres) y 10 sin TDAH (cinco hombres y cinco mujeres) por el Instituto Nacional de Rehabilitación (INR), en la ciudad de México, basados en pruebas clínicas estandarizadas DSM-IV [18].

\subsection{Estimulación.}

Las estimulaciones que se ocuparon para cada uno de los estudios fueron de tipo visual mediante la aplicación de un tren de veinte pulsos de diez foto-estimulaciones con flash cada uno, separados uno del otro por intervalos de tiempo de duración aproximada de 0.2 segundos, es decir, la duración del ancho de cada tren de estimulación fue de aproximadamente 2 segundos, lo anterior es mostrado gráficamente en la figura 5.2:

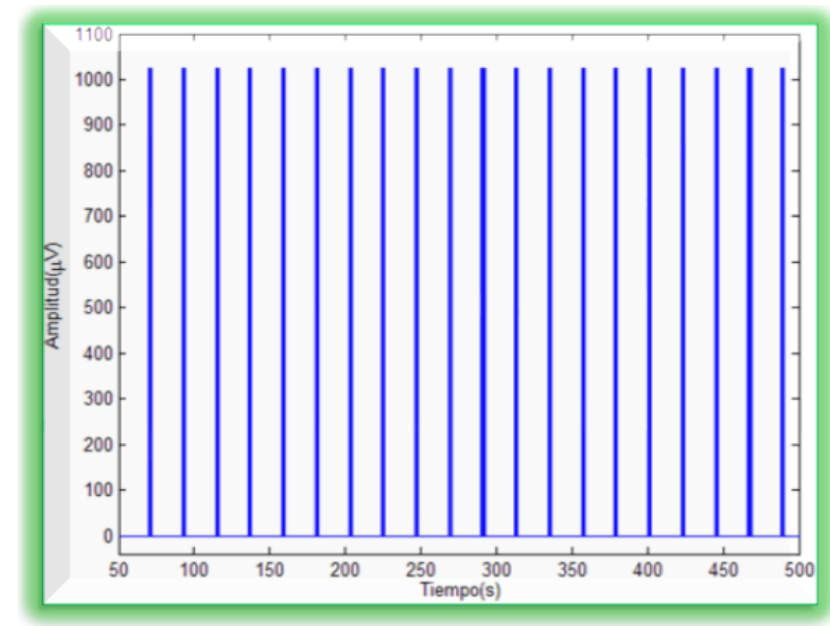

$a$

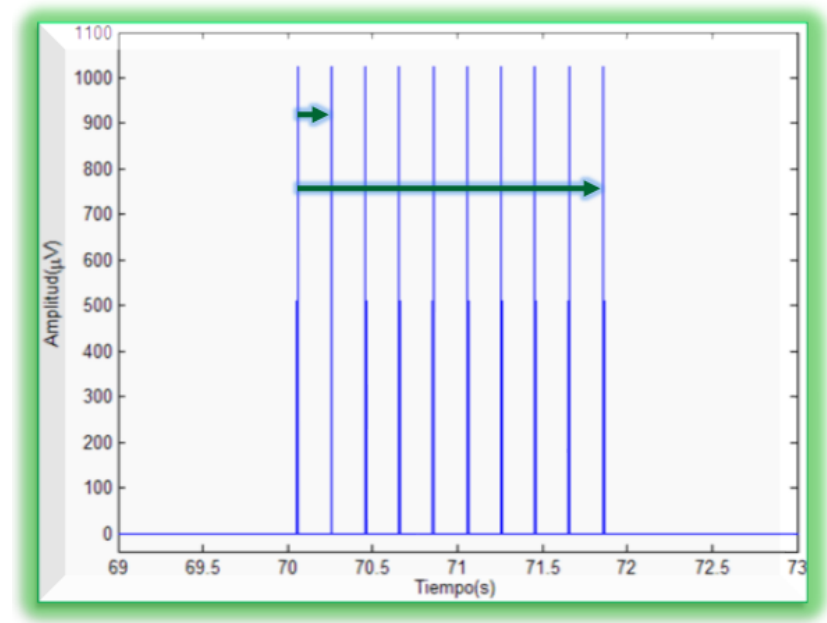

$b$

Figura 5.2:

a)Tren de veinte foto-estimulación completas

b)Primeros diez flashes correspondietes al primer pulso de foto-estimulación.

\subsection{Adquisición e Instrumentación.}

Para llevar a cabo el proceso de adquisición de los registros electroencefalográficos de las poblaciones estudiadas, se utilizó como base el sistema internacional de colocación de electrodos 10/20, ocupando para el registro únicamente diez canales monopolares, cinco de los cuales correspondieron al hemisferio derecho: $F_{p 2}, F_{4}, C_{4}, P_{4}, O_{2}$ y los restantes al izquierdo: $F_{p 1}, F_{3}, C_{3}$, $P_{3}$ y $O_{1}$.

Posteriormente, ya fuera de línea, se obtuvieron por sustracción digital de cada par de electrodos localizados sobre el mismo hemisferio, ocho nuevas señales bipolares hemiesféricas; cuatro por hemisferio tal y como es mostrado en la figura 5.3. 


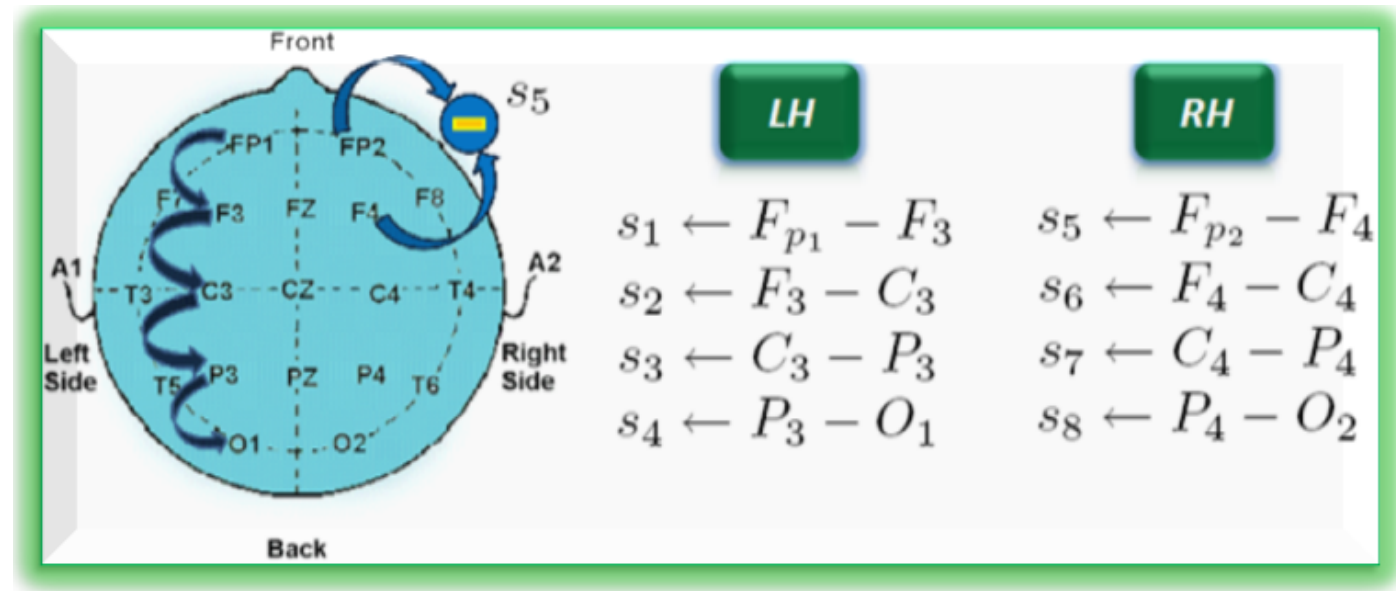

Figura 5.3:

Representación esquemática del proceso de adquisición de los registros electroencefalográficos.

La frecuencia de muestreo $f s$ fue de $256 \mathrm{~Hz}$, filtrando cada una de las señales adquiridas, con un filtro pasa banda digital cuyas frecuencias de corte fueron $f_{H}=0,5 \mathrm{~Hz}$ y $f_{L}=40 \mathrm{~Hz}$.

\subsection{Condiciones de registro.}

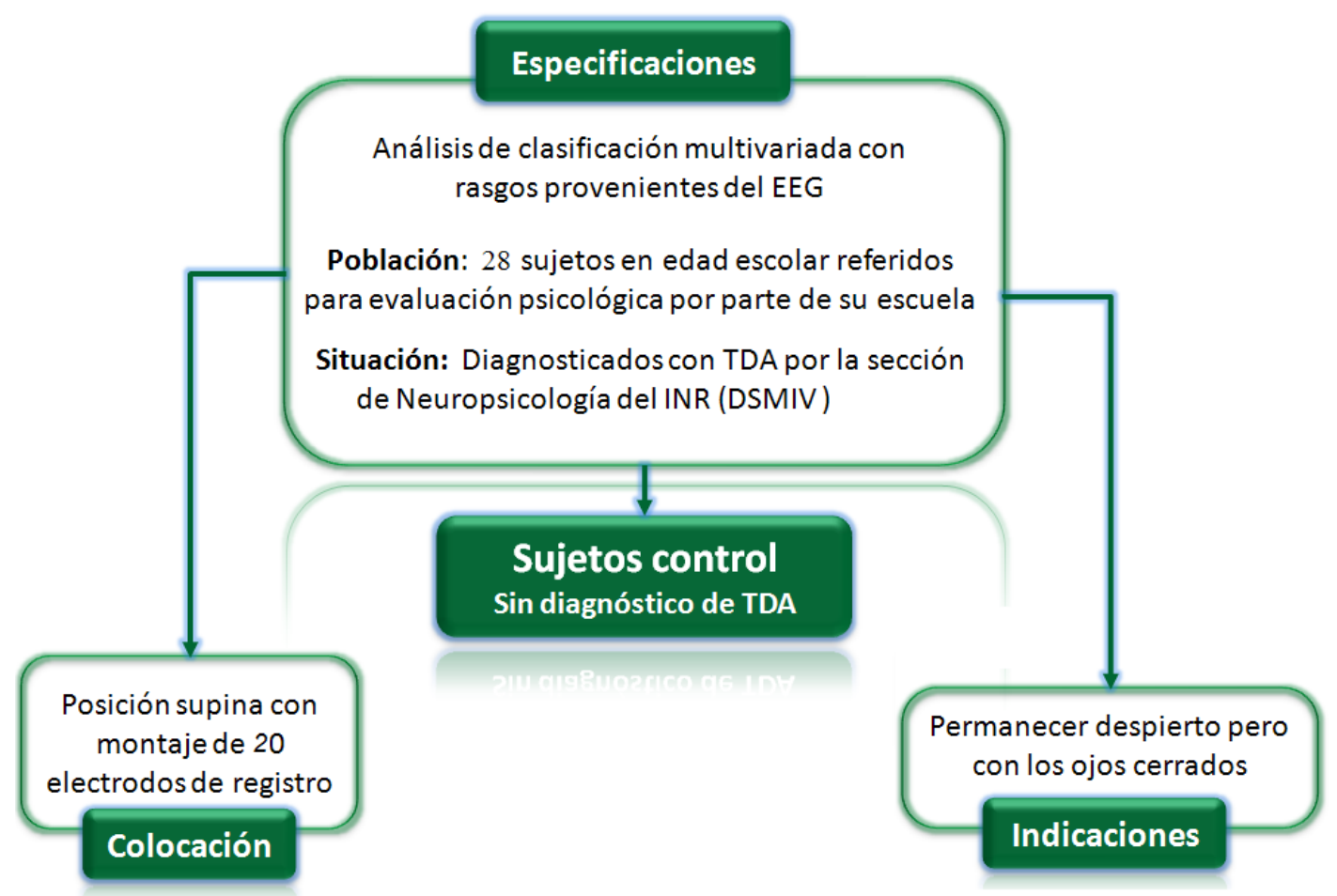

Figura 5.4:

Representación esquemática de las condiciones establecidas en el proceso de adquisición.

En cada uno de los estudios, los sujetos se colocaron en posición supina montándoles los veinte electrodos de registro e indicándoles que permanecieran despiertos pero manteniendo sus ojos cerrados y además solicitándoles que oprimieran un boton, que sirvió como indicador de respuesta y atención del sujeto registrado. 


\subsection{Pre-Procesamiento.}

Cada una de las ocho señales bipolares obtenidas fueron tratadas digitalmente para su posterior análisis, básicamente fueron sometidas a los procesos siguientes.

\subsubsection{Filtrado.}

Posterior al registro, fueron separados los ritmos cerebrales de interés del EEG adquirido, diseñando para ello un banco de filtros digitales FIR de orden cien de Fase lineal por el método de ventanas [13]. El proceso anterior se realizó con el objetivo principal de poder analizar cada una de las señales adquiridas en las bandas de frecuencia lenta: $\delta$ (Filtro Pasa Bajas con frecuencia de corte $f_{o}=4 \mathrm{~Hz}$ ) y $\theta$ (Filtro Pasa Banda con frecuencias de corte baja $f_{L}=4 \mathrm{~Hz}$ y alta $f_{H}=8 \mathrm{~Hz}$ ) así como en las de frecuencia rápida: $\alpha$ (Filtro Pasa Banda con frecuencias de corte baja $f_{L}=8 \mathrm{~Hz}$ y alta $f_{H}=13 \mathrm{~Hz}$ ) y $\beta$ (Filtro Pasa Altas con frecuencia de corte $f_{o}=13 \mathrm{~Hz}$ ). Teniendo hasta este punto 32 señales con información de las distintas ubicaciones lobulares a diferentes ritmos.

\subsubsection{Segmentación.}

Al tener las señales, correspondientes a cada uno de los ritmos cerebrales, para cada una de las señales bipolares se realizó una partición de las mismas, generando para ello un proceso de ventaneado que permitió procesar las señales en intervalos de tiempo de interés.

En particular, fueron observadas las fluctuaciones existentes para cada una de las veinte repeticiones de la foto-estimulación así como para la ubicación temporal de la misma, es decir, si el segmento de señal se encontraba previo al estímulo, posterior al estímulo o durante éste, terminando en total con 1920 segmentos de señal por sujeto.

El proceso antes descrito se muestra de forma esquemática en la figura 5.5.
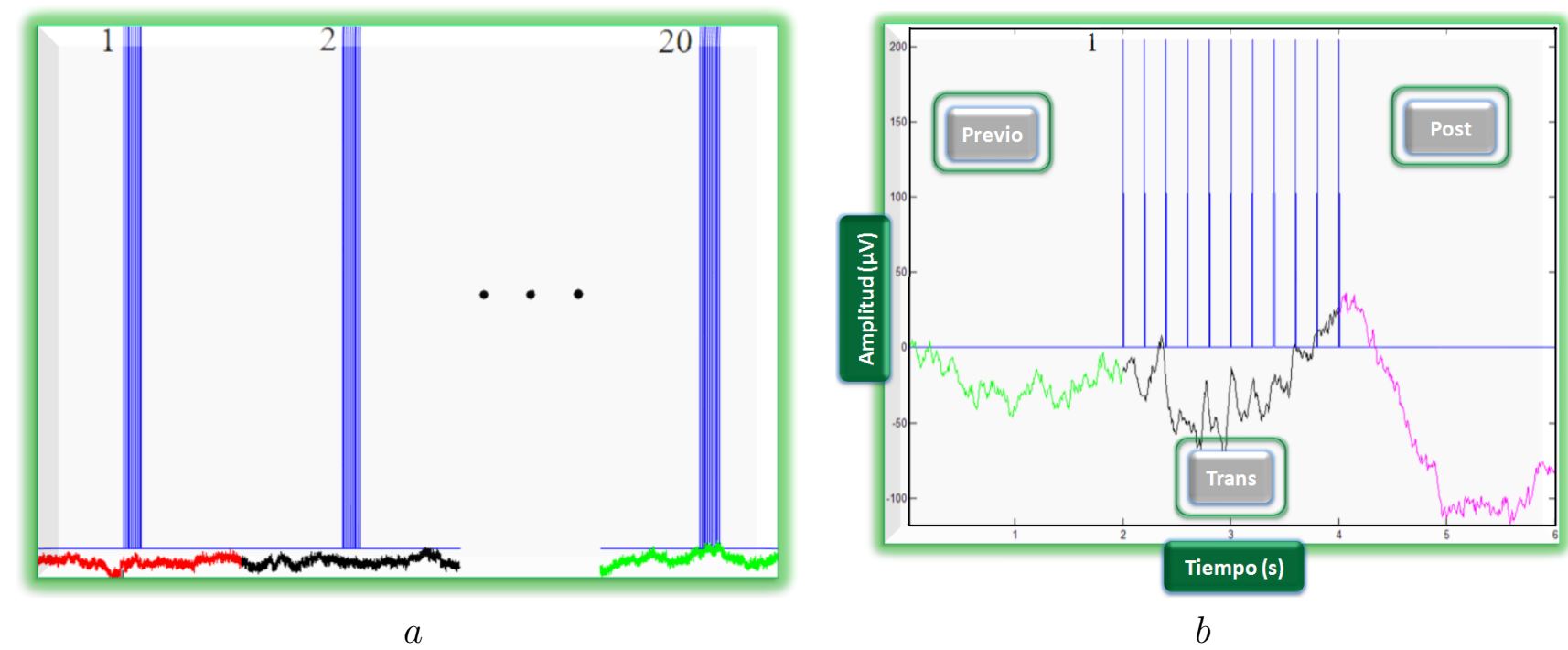

Figura 5.5:

Representación esquemática del proceso de segmentación de la señal para cada una de las veinte repeticiones de la estimulación (a), así como para el de la señal previa, durante y posterior a la primera estimulación $(b)$. 


\subsection{Extracción de características inmersas en las señales Electroencefalográficas.}

El problema general de extracción de características inmersas en las señales electroencefalográficas registradas se resolvió por dos caminos:

1. Mezclando un análisis espectral y un proceso de segmentación de estas señales mediante ventanas de tiempo dependientes a la foto-estimulación aplicada, asumiendo en este proceso estacionaridad en tiempos cortos, estudiando los contenidos energéticos en bandas adoptadas por la clínica.

2. Por la aplicación de un análisis multiresolución mediante una descomposición en paquetes Wavelet completa analizando en este caso, la energía asociada a cada conjunto de coeficientes calculados.

\subsubsection{Rasgos Frecuenciales}

Básicamente, las señales de EEG pueden ser caracterizadas por su contenido espectral, de esta manera, considerando que el contenido en frecuencia en las distintas ubicaciones espaciales del cerebro caracterizan la actividad eléctrica promedio en dichas posiciones, se planteó estudiar el contenido frecuencial de cada una de las señales cerebrales registradas para las diferentes ubicaciones donde fue aplicada la fotoestimulación mencionada con anterioridad así como donde no fue aplicada ya que la falta de estimulación modifica la actividad eléctrica cerebral, ésto, debido al interes de combinar la información de tiempo y frecuencia en los vectores de características.

Las características espectrales de cualquier proceso aleatorio como lo es, en este caso, el electroencefalograma, se obtienen calculando la tranformada de Fourier de su función de autocorrelación, si ésta es discreta y queda denotada por $\gamma_{x x}(n)$ entonces;

$$
S_{x x}\left(e^{j \omega_{k}}\right)=\sum_{n=0}^{N-1} \gamma_{x x}(n) \cdot e^{-j \omega_{k} n} \quad \forall \quad k=0,1, \cdots, N-1 \quad \text { con } \quad \omega_{k}=\frac{2 \pi k}{N}
$$

Por otro lado, la transformada discreta inversa de Fourier sería:

$$
\gamma_{x x}(n)=\frac{1}{N} \sum_{k=0}^{N-1} S_{x x}\left(e^{j \omega_{k}}\right) \cdot e^{j \omega_{k} n} \quad \forall n=0,1, \cdots, N-1
$$

Observando de (5.2) que para valores de lag cero se tiene

$$
\gamma_{x x}(0)=\frac{1}{N} \sum_{k=0}^{N-1} S_{x x}\left(e^{j \omega_{k}}\right)=E\left(X_{n}^{2}\right) \geq 0
$$

La relación mostrada en (5.3) representa la potencia media del proceso aleatorio, que es el área bajo $S_{x x}\left(e^{j \omega_{k}}\right)$, con ésto se desprende que $S_{x x}\left(e^{j \omega_{k}}\right)$ es la distribución de potencia en función de la frecuencia discreta. Por esta razón $S_{x x}\left(e^{j \omega_{k}}\right)$ se conoce como la densidad espectral de potencia DEP del proceso aleatorio. 
Para estimar la DEP, se ocupó el método de Welch [13]: Promediado de periodogramas modificados, que permite el solapamiento en los segmentos de datos además de enventanarlos antes de calcular el periodograma. El resultado es un periodograma modificado definido por la relación siguiente:

$$
\tilde{P}_{x x}^{i}\left(\omega_{k}\right)=\frac{1}{M U}\left|\sum_{n=0}^{M-1} x_{i}(n) w(n) e^{-j \omega_{k} n}\right|^{2}
$$

Donde $U$ es un factor de normalización para la potencia de la función ventana.

La estimación de Welch de la potencia espectral es el promedio de este conjunto de periodogramas modificados con estructura como en 5.5 .

$$
P_{x x}^{W}\left(\omega_{k}\right)=\frac{1}{L} \sum_{i=0}^{L-1} \tilde{P}_{x x}^{i}\left(\omega_{k}\right)
$$

Una vez realizada la estimación de la DEP para cada uno de los segmentos a estudiar, se procedió a calcular la potencia media del proceso por integración numérica vía regla del trapecio siendo éstos los índices absolutos $P_{A}$, por otro lado, los índices relativos $P_{R}$ fueron obtenidos dividiendo cada índice absoluto por la potencia media total $P_{T}$ de la señal, es decir

$$
P_{R_{b}}=\frac{P_{A_{b}}}{P_{T}} \quad \forall \quad b=\{\delta, \theta, \alpha, \beta\}
$$

De esta manera, los vectores de rasgos frecuenciales para cada una de las ondas cerebrales tiene la forma mostrada en 5.7

$$
x_{b}=\left\{P_{b_{i, j, k}}\right\} \in \mathbb{R}^{480} \quad \forall \quad \begin{gathered}
i=\text { anterior, durante, posterior } \\
j=1,2, \cdots, 20 \\
k=1,2, \cdots, 8
\end{gathered}
$$

Donde $i$ denota la ubicación temporal (anterior, durante o posterior al estímulo aplicado), $j$ corresponde a la repetición para cada una de estas estimulaciones aplicadas, $k$ es la señal bipolar adquirida, $P$ se refiere al índice de potencia calculado y $b$ representa los cuatro ritmos cerebrales estudiados.

Por consiguiente, si la población que fue estudiada se compusó en total de 28 sujetos entonces, la matriz de rasgos por sujeto generada, bajo este análisis, para cada una de las ondas cerebrales a clasificar presentó la estructura mostrada en (5.8)

$$
s D_{b}=\left\{x_{b_{1}}^{T}, x_{b_{2}}^{T}, \cdots, x_{b_{28}}^{T}\right\} \in \mathbb{R}^{[28 * 480]}
$$

De esta manera, se generaron en total cuatro matrices de rasgos por sujeto con estructura mostrada en (5.8), una por cada ritmo cerebral estudiado, que caracterizaron a cada uno de los sujetos que conformaron la población. 


\subsubsection{Rasgos Wavelet}

Los Wavelets han sido usados extensamente en la codificación de imágenes brindando una representación compacta y consistente de buena precisión ya que poseen la habilidad de capturar el comportamiento global de alguna señal, en una expansión de baja resolución, y moverse progresivamente en más detalle como sea necesario suministrando así, una herramienta flexible para una descomposición eficiente.

A muy grandes rasgos, el Análisis MultiResolución (AMR) o aproximación multiescala consiste en aproximar una función $f(t)$, que en este caso consistió en cada una de las señales bipolares registradas, mediante diferentes niveles de resolución; el AMR puede ser visto como una secuencia de subespacios anidados que satisfacen relaciones de similitud en tiempo/espacio y escala/frecuencia, además de relaciones de completitud y regularidad [19].

\section{Paquetes Wavelet en la extracción de características cerebrales}

Debido a que las señales estudiadas reflejaban la actividad eléctrica promedio del cerebro, para diferentes regiones del mismo, el ancho de banda mínimo de interes respecto al detalle para el contenido espectral correspondió al rango de frecuencias del ritmo más lento; $\delta$.

Al considerar esto, fue posible establecer el nivel al cual se llevó a cabo la descomposición completa en paquetes wavelet, siendo éste calculado mediante la relación mostrada en 5.9 .

$$
\omega_{o}=\frac{2 \cdot \pi \cdot f_{o}}{f_{s}}=\frac{2 \pi \cdot 4}{256}=\frac{2 \pi \cdot 2^{2}}{2^{8}}=\frac{\pi}{2^{5}} \Rightarrow n=5
$$

Donde $\omega_{o}$ y $f_{o}$ corresponden a las frecuencias de corte digital y analógica respectivamente mientras que $f_{s}$ representa a la frecuencia de muestreo con la que se adquirieron las señales.

De esta manera se encontró que el nivel de descomposición necesario, para explorar las señales incluyendo el contenido espectral de la onda cerebral más lenta en el detalle de resolución, correspondía a $n=5$ tal y como se observa en la respuesta en frecuencia generada por la descomposición wavelet (figura 5.6). Al calcular dicha descomposición se dispuso de 32 conjuntos de coeficientes para cada señal bipolar como es mostrado en el arbol de análisis $(a)$ de la misma figura y denotados por

$$
\left[C_{1}, C_{2}, C_{3}, \cdots, C_{32}\right]=\left[\left\{\begin{array}{c}
c_{1,1} \\
c_{1,2} \\
\vdots \\
c_{1, k}
\end{array}\right\},\left\{\begin{array}{c}
c_{2,1} \\
c_{2,2} \\
\vdots \\
c_{2, k}
\end{array}\right\},\left\{\begin{array}{c}
c_{3,1} \\
c_{3,2} \\
\vdots \\
c_{3, k}
\end{array}\right\}, \cdots,\left\{\begin{array}{c}
c_{32,1} \\
c_{32,2} \\
\vdots \\
c_{32, k}
\end{array}\right\}\right] \in \mathbb{R}^{k x 32}
$$

A cada uno de estos conjuntos de coeficientes $C_{i}$, le fue calculado su medida de energía, como en 5.11, para de esta manera contar con un solo vector de rasgos por señal bipolar, es decir, cada sujeto tuvo asociado ocho vectores de rasgos wavelet, uno por cada señal bipolar registrada, que reflejaban información de energía para cada banda de frecuencia respectiva.

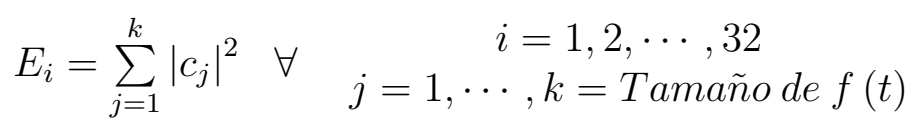



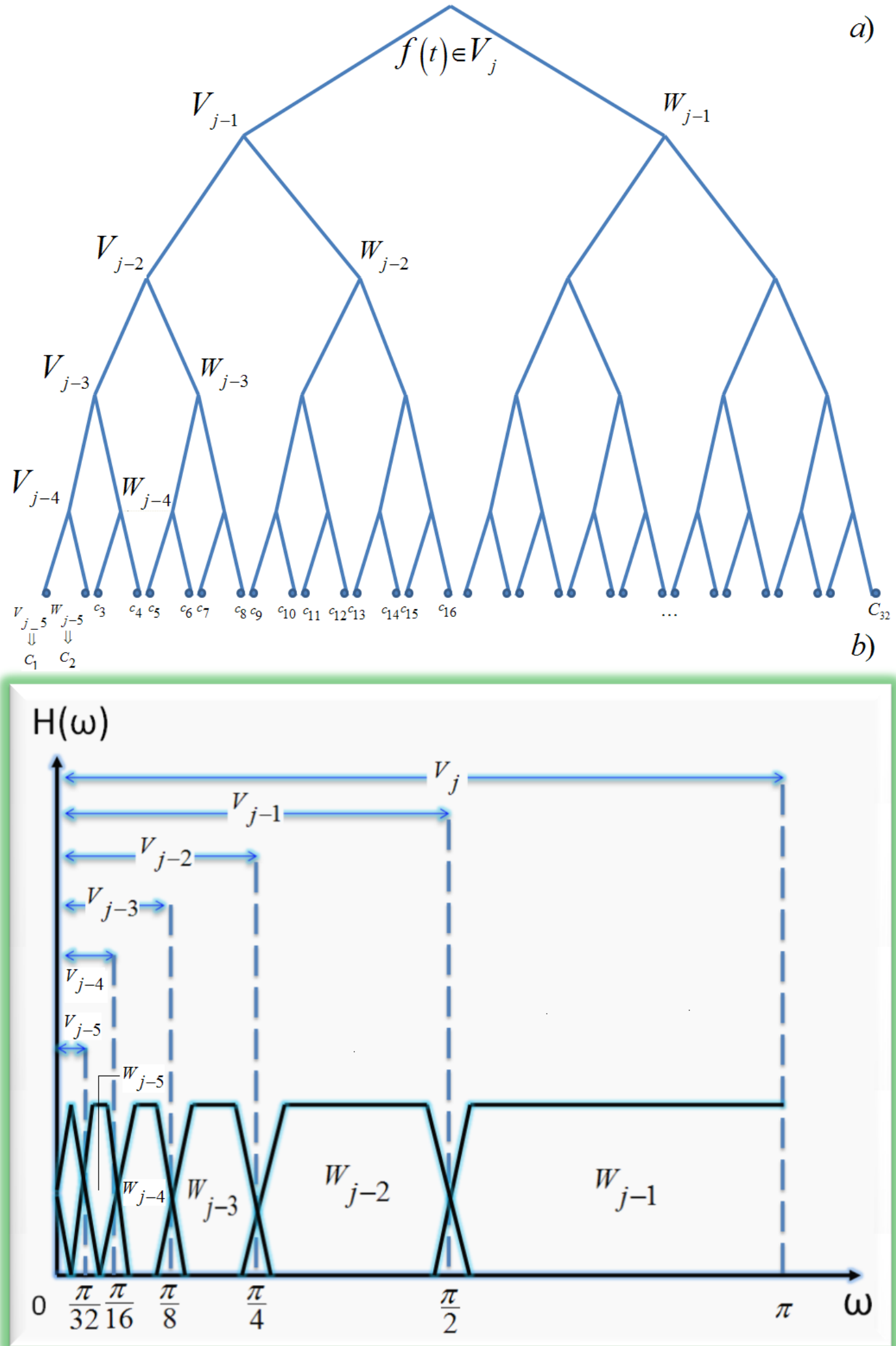

Figura 5.6: Análisis multiresolución mediante descomposición completa en paquetes wavelet. $V$ y $W$ son espacios formados por conjuntos de funciones wavelet; $V_{j}$ y $V_{j-1}$ son subespacios adyacentes mientras que $V_{j-1}$ y $W_{j-1}$ son subespacios ortogonales a) Arbol de análisis a un nivel de descomposición igual a cinco.

b) Respuesta en frecuencia generada por la descompoción aplicada: Banco de filtros con ancho de banda igual a $4 \mathrm{~Hz}$ 


\section{Capítulo 6}

\section{Arquitectura de análisis}

Genios sin estudios es como plata en la mina. BENJAMIN FRANKLIN

En la Fig. 6.1 se presenta la propuesta de arquitectura de análisis aplicada para los conjuntos de rasgos frecuenciales extraídos que básicamente se compone de una etapa de registro de señales cerebrales, otra de extracción de rasgos y dos niveles de clasificación con mapas auto-organizados de 10x10 nodos cada uno.

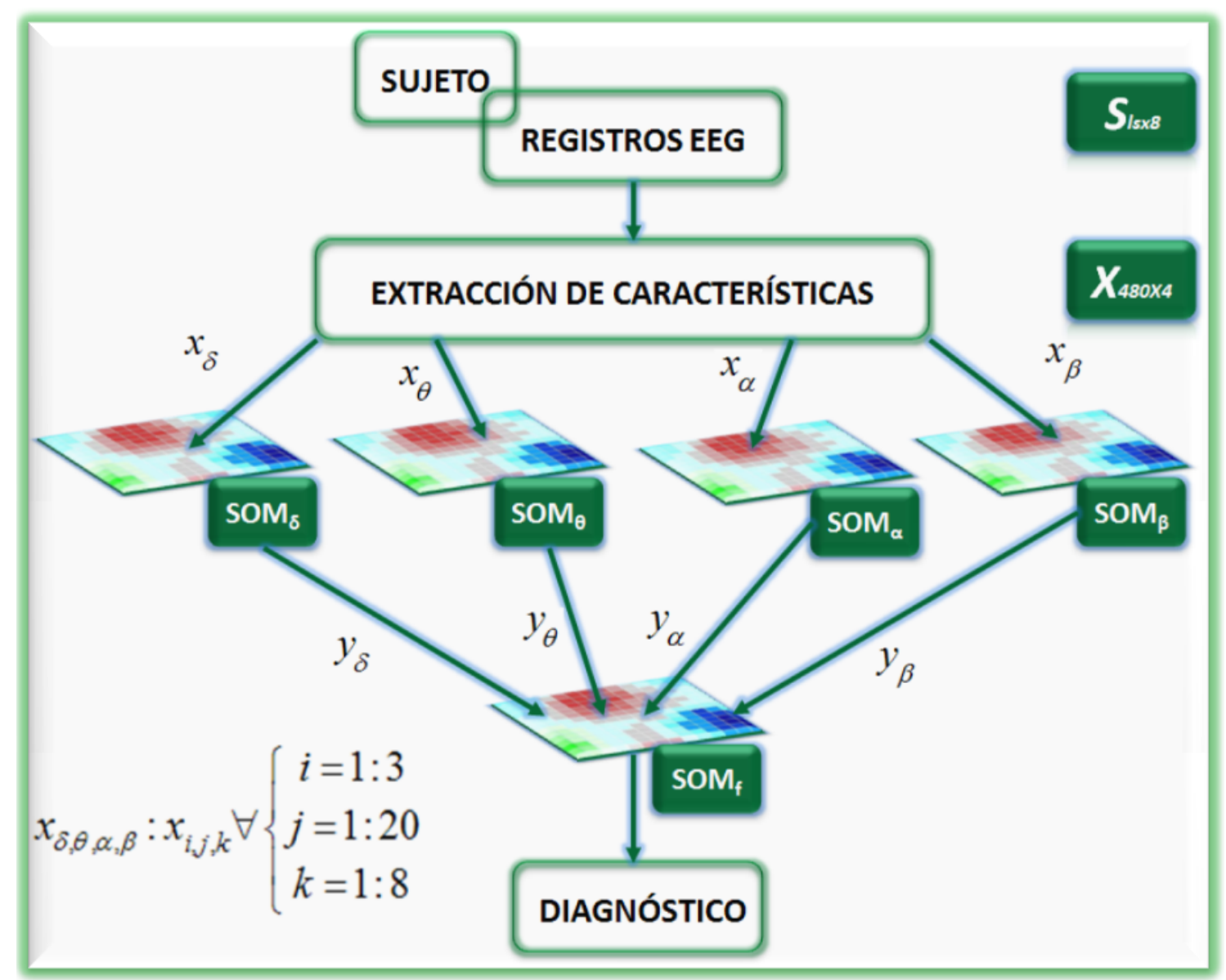

Figura 6.1

Diagrama de la arquitectura propuesta para el análisis de los datos; registro de EEG, extracción de rasgos, búsqueda de patrones por onda cerebral para los rasgos frecuenciales y posterior combinación de los patrones encontrados en el nivel uno para la formación del mapa clasificador final. 
Para el caso de los rasgos wavelet la única modificación en la arquitectura se presenta en el nivel uno donde se generaron mapas por cada señal bipolar registrada y no por cada ritmo cerebral como lo mostrado para los rasgos frecuenciales.

El trabajo realizado por cada uno de estos niveles es el que se explica a continuación:

- El primer nivel de análisis se encargó de generar mapas $\left(S O M_{\delta}, S O M_{\theta}, S O M_{\alpha}, S O M_{\beta}\right)$ entrenados por los rasgos de potencia en banda para cada uno de los ritmos cerebrales y mapas $\left(S O M_{F_{p 1}-F_{3}}, S O M_{F_{3}-C_{3}}, S O M_{C_{3}-P_{3}}, S O M_{P_{3}-O_{1}}, S O M_{F_{p 2}-F_{4}}, S O M_{F_{4}-C_{4}}, S O M_{C_{4}-P_{4}}\right.$, $S O M_{P_{4}-O_{2}}$ ) entrenados por los rasgos wavelet de energía por cada señal bipolar registrada respectivamente.

- Mientras que en el segundo nivel de análisis, después de entrenar a los primeros mapas, los patrones formados en el nivel anterior fueron aprendidos de la forma siguiente: para cada vector de entrada $x_{b i}$, donde $i$ denota al $i$-ésimo sujeto, se mapeó la coordenada espacial correspondiente al nodo activado en dicho mapa, tomándolo como una salida $y_{b i}$ del mismo mapa.

El conjunto de todas las salidas $\left\{y_{b 1}, y_{b 2}, \cdots, y_{b n}\right\}$ para los cuatro u ocho mapas, según los rasgos analizados, del nivel anterior son subsecuentemente combinados para posteriormente ser usados como un único vector de rasgos a ser aprendido por un sólo $S O M_{f}$ en el nivel dos de la arquitectura, es decir, para el conjunto de rasgos frecuenciales se tuvo

$$
y=\left\{\begin{array}{llll}
y_{\alpha} & y_{\beta} & y_{\theta} & y_{\delta}
\end{array}\right\}=\left\{\begin{array}{cccl}
y_{\alpha_{1}} & y_{\beta_{1}} & y_{\theta_{1}} & y_{\delta_{1}} \\
y_{\alpha_{2}} & y_{\beta_{2}} & y_{\theta_{2}} & y_{\delta_{2}} \\
\vdots & \vdots & \vdots & \vdots \\
y_{\alpha_{n}} & y_{\beta_{n}} & y_{\theta_{n}} & y_{\delta_{n}}
\end{array}\right\} \in \mathbb{R}^{[n * 4]}
$$

Al finalizar el entrenamiento en el nivel dos y disponer del $S O M_{f}$ se tomó su codebook final para etiquetarlo con fines de clasificación mediante un proceso en dos pasos:

- Primero, el codebook fue agrupado usando el algoritmo $k$-means, variando el valor del parámetro $k$ y eligiendo al final la $k$ que arrojó el valor mínimo para la medida de error (suma del error cuadrático), este agrupamiento determinó grupos de nodos con codebooks similares determinando así las fronteras óptimas sobre el mapa final de los clusters encontrados durante todo el proceso.

- Segundo, la probabilidad de que un cluster representara un caso positivo de TDAH fue calculado por el conteo de las activaciones dentro de dicho cluster cuando el mapa es presentado al conjunto de entrenamiento etiquetado para finalmente establecer arbitrariamente las áreas de probabilidad máxima, moderada y mínima de presentar la enfermedad. 


\subsection{Proceso de validación del mapa clasificador}

Para llevar a cabo este proceso se diseñó una validación cruzada a seis vías, es decir, el conjunto total de estudio se particionó aleatoriamente en seis subconjuntos $\left\{G_{1}, G_{2}, G_{3}, G_{4}, G_{5}, G_{6}\right\}$ cada uno constituido de tres niños con TDAH y dos niños sin TDAH, tanto para los rasgos frecuenciales como para los rasgos wavelet.

Posterior a esta partición se generaron dos nuevos grupos formados por la combinación de los primeros, para así construir al conjunto de entrenamiento denotado por $s D e$, constituido por cinco de estos subconjuntos, y al conjunto de validación denotado por $s D v$, constituido por el subconjunto restante, repitiendo este proceso para todas las posibles combinaciones entre los seis subconjuntos tal y como se muestra en 6.2

$$
\begin{aligned}
& s D e_{1}=\left\{G_{1}, G_{2}, G_{3}, G_{4}, G_{5}\right\} \leftrightarrow s D v_{1}=\left\{G_{6}\right\} \\
& s D e_{2}=\left\{G_{1}, G_{2}, G_{3}, G_{4}, G_{6}\right\} \leftrightarrow s D v_{2}=\left\{G_{5}\right\} \\
& s D e_{3}=\left\{G_{1}, G_{2}, G_{3}, G_{5}, G_{6}\right\} \leftrightarrow s D v_{3}=\left\{G_{4}\right\} \\
& s D e_{4}=\left\{G_{1}, G_{2}, G_{4}, G_{5}, G_{6}\right\} \leftrightarrow s D v_{4}=\left\{G_{3}\right\} \\
& s D e_{5}=\left\{G_{1}, G_{3}, G_{4}, G_{5}, G_{6}\right\} \leftrightarrow s D v_{5}=\left\{G_{2}\right\} \\
& s D e_{6}=\left\{G_{2}, G_{3}, G_{4}, G_{5}, G_{6}\right\} \leftrightarrow s D v_{6}=\left\{G_{1}\right\}
\end{aligned}
$$

Para cada conjunto de entrenamiento formado $s D e_{i}$, se aplicó la arquitectura de análisis presentada en la sección anterior una vez disponiendo del $S O M_{f i}$, las muestras etiquetadas de $s D v_{i}$ son sometidas a los mapas multicapa para observar los nodos activados y determinar con ello, la eficiencia del soporte brindado por el $S O M_{f i}$ estableciendo para ello, el valor medio de la tasa de acierto $\overline{t_{a}}$ asi como la variación de la misma $s_{t_{a}}$.

\subsection{Procesamiento del mapa entrenado}

Cada uno de los mapas entrenados mediante la arquitectura mostrada en la figura 6.1, fue posteriormente procesado de la manera siguiente:

1. Se decidió tomar al codebook final y obtener su descomposición en componentes principales (PCA, por sus siglas en ingles Principal Component Analysis), en particular se buscó observar la distribución de los datos en un espacio bidimensional por lo que se graficó cada par de coordenadas de los dos componentes principales calculados y sobre este espacio de datos, se montó la red SOM generada durante el entrenamiento asociando para cada uno de los nodos una codificación en color que permitió observar la posible generación de clusters.

La figura 6.2 muestra este primer proceso, los puntos marcados con cruces se obtuvieron al graficar las coordenadas de los dos componentes principles generados después del análisis PCA mientras que los puntos marcados en color, corresponden a los nodos o neuronas del mapa final generado después del entrenamiento los cuales, trataran de modelar a los primeros puntos.

La idea fue que mediante la asignación de una codificaión en color sobre el mapa se pudieran encontrar regiones sobre éste que representaran conjuntos de datos estudiados, por ejemplo, 
de la gráfica mostrada en la figura 6.2 rápidamente pueden encontrarse tres grupos de nodos que representan a toda la población (los nodos naranja, los verdes y los morados) sin embargo, muy probablemente existen más grupos entre éstos ya que como puede observarse, el color de los nodos se ven modificados en dirección al centro del mapa.

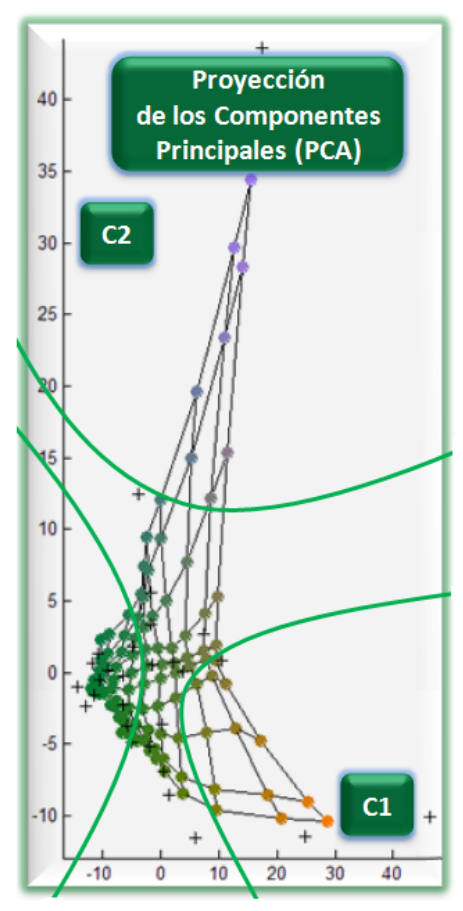

Figura 6.2:

Ejemplo del análisis de los componentes principales del codebook final .

2. Posterior a esta primera aproximación en la visualización del mapa, se generó un histograma plano donde se observó tanto la ubicación sobre el espacio de red del nodo como el número de veces que éste fue activado durante el aprendizaje maquinal (proceso por el cual, la red se organizó bajo el algoritmo de aprendizaje mostrado en la figura 4.4 del capítulo 4), asignando igualmente para cada uno de los nodos del mapa activados una codificación en color que permitió observar más claramente los clusters generados. En la figura 6.3 se ilustra este segundo proceso.
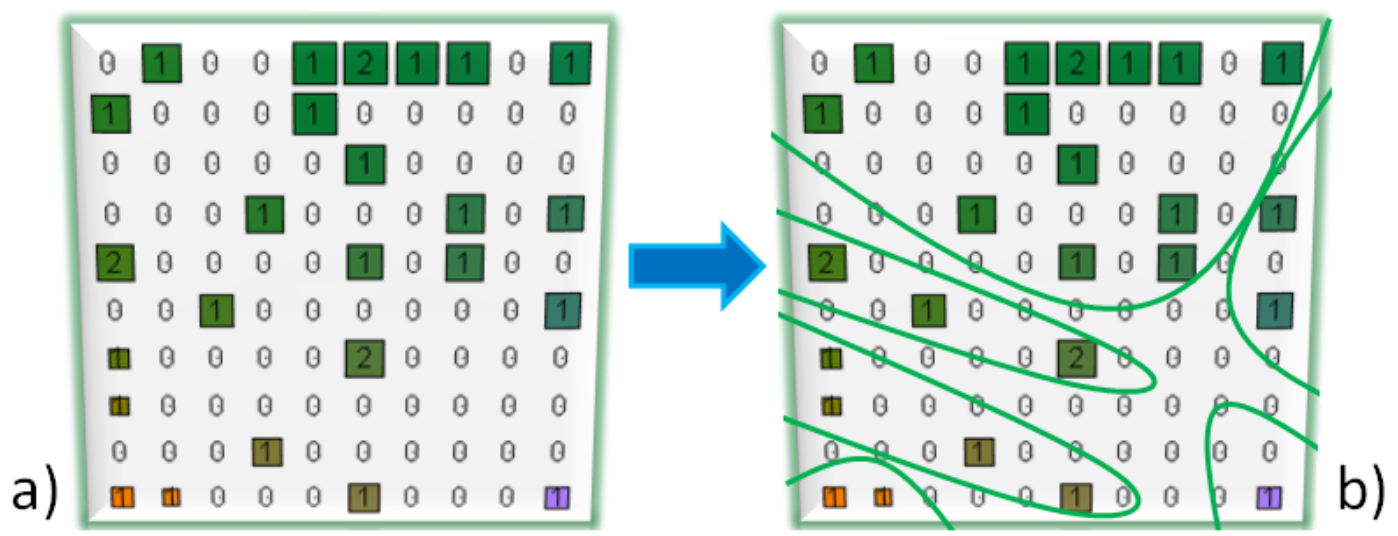

Figura 6.3:

Ejemplo de la generación del histograma plano sobre el espacio del mapa. 
Con esta segunda visualización además de observar lo ya mencionado sobre las áreas del mapa con mayor activación, se pueden encontrar con mayor facilidad las regiones del mapa que representan a ciertos conjuntos de datos debido al despliegue aplanado del mapa final y al color asignado a cada uno de los nodos, observando en esta ocasión seis posibles clusters para el mismo mapa (gráfica b de la figura 6.3, nodos con color semejante conforman un mismo cluster).

3. Finalmente para poder garantizar la existencia de límites o fronteras, de los posibles clusters generados, perfectamente marcados sobre el mapa final entrenado se tomó al codebook resultante y se sometió al algoritmo de agrupamiento $k$-means, eligiendo el parámetro $k$ que arrojara el valor mínimo para la suma del error cuadrático, generando con ello las fronteras óptimas sobre el mapa de cada uno de los clusters y por último, se asignó color a cada región delimitada. En la figura 6.4 se muestra este tercer proceso.

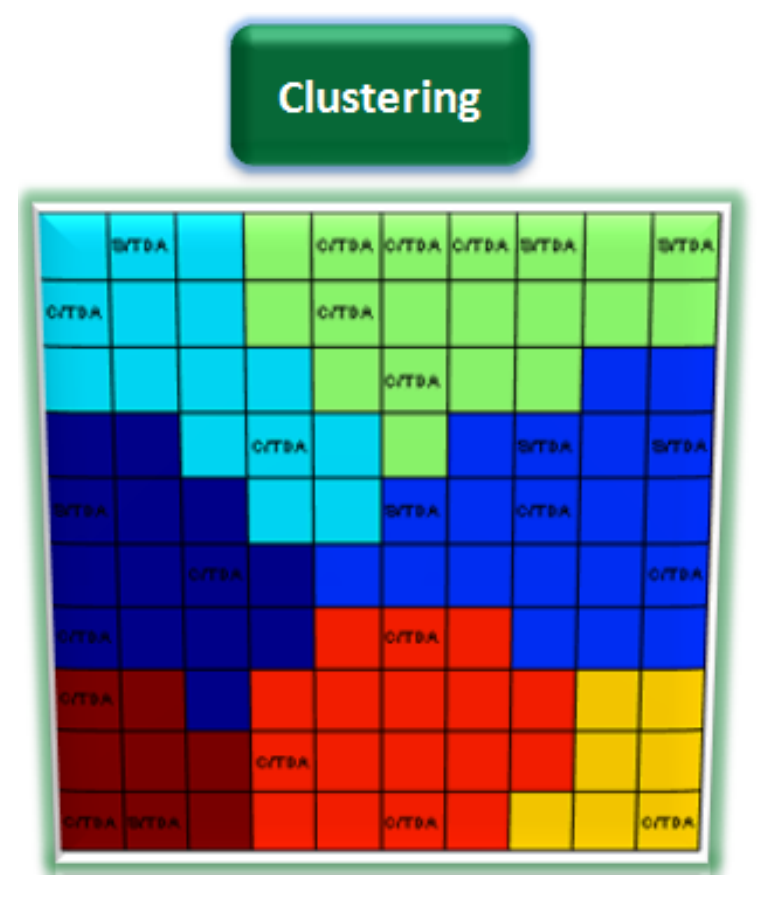

Figura 6.4:

Ejemplo del análisis cluster realizado para la generación de las fronteras óptimas sobre el mapa clasificador.

Con este cuarto proceso, se pudó definir de forma precisa los nodos que conformaron a los clusters encontrados. Para la gráfica ejemplo de la figura 6.4 se observan siete clusters delimitados perfectamente por las fronteras calculadas en este paso y que a diferencia de la gráfica ejemplo de la figura 6.3 no se pudó delimitar de tal manera.

4. Una vez segmentado cada mapa, fueron definidas sobre éstos tres regiones de probabilidad (máxima, moderada y mínima) de presentar TDAH siempre en función de los nodos activados por cada conjunto de entrenamiento respectivo.

Por ejemplo si en el cluster azul de la figura 6.4 solamente se activaron tres nodos, dos de los cuales presentaron etiqueta positiva de TDAH, entonces la probabilidad $p$ de presentar TDAH para este cluster particular fue de $\frac{2}{3} \approx 0,66$. 


\subsection{Interpretación del mapa final}

Para ejemplificar de mejor manera la interpretación del mapa clasificador final generado por la aplicación de los procesos descritos en la sección anterior, obsérvese el mapa ejemplo mostrado en $(a)$ de la figura 6.5 en el cual aparecen siete clusters, los cuales agrupan a un número finito de nodos que poseen características en común (determinadas de forma automática durante el proceso de aprendizaje del mapa), sólo algunos de estos nodos fueron activados durante el proceso de entrenamiento del mapa y corresponden a aquellos nodos que presentan alguna de las dos posibles etiquetas.

- Etiqueta positiva $\mathrm{TDAH}^{+} \rightarrow \mathrm{C} / \mathrm{TDA}$

- Etiqueta negativa $\mathrm{TDAH}^{-} \rightarrow \mathrm{S} / \mathrm{TDA}$

Para poder definir cada una de las regiones de probabilidad sobre el mapa final entrenado fue tomado como base a este conjunto de nodos activados, básicamente se buscó determinar la probabilidad de presentar TDAH para cada uno de estos clusters, calculando para ello el cociente entre el número de nodos activados con etiqueta positiva y el número total de nodos activados para cada cluster.

De esta forma, la regiones de probabilidad de presencia de la enfermedad quedaron definidas de la forma siguiente:

- Región de máxima probabilidad:

Definida por todos los clusters que presentaron una probabilidad $p \geq 0,75$

- Región de probabilidad moderada:

Definida por todos los clusters con probabilidades entre $0,5<p<0,75$

\section{- Región de probabilidad mínima:}

Definida por todos los clusters con probabilidades $p \leq 0,5$

Estas regiones de probabilidad fueron ocupadas siempre y cuando la activación, producida por los sujeto de validación, fuera en los nodos que no presentaran ninguna de las posibles etiquetas (C/TDA o S/TDA) definiendo para este caso, la probabilidad que presenta dicho sujeto de tener la enfermedad. Por otra parte, cuando los sujetos activaron nodos etiquetados las regiones de probabilidad pasaron a segundo plano y la etiqueta tendría mayor valor en el soporte.

Con lo anterior se aseguró que cada región de probabilidad definida fuera proporcional al número de sujetos diagnosticados con TDAH, es decir, la región de probabilidad máxima contendría la mayoría de los sujetos con TDAH ocupados para entrenar al mapa y en contra parte, la mínima presentaría la minoría de estos sujetos tal y como lo muestra el histograma en (b) de la figura 6.5 . 
(a) Mapa ejemplo con los clusters encontrados y etiquetas de los nodos activados durante el entrenamiento. Los clusters dentro de los circulos marcados indican regiones de alta probabilidad.

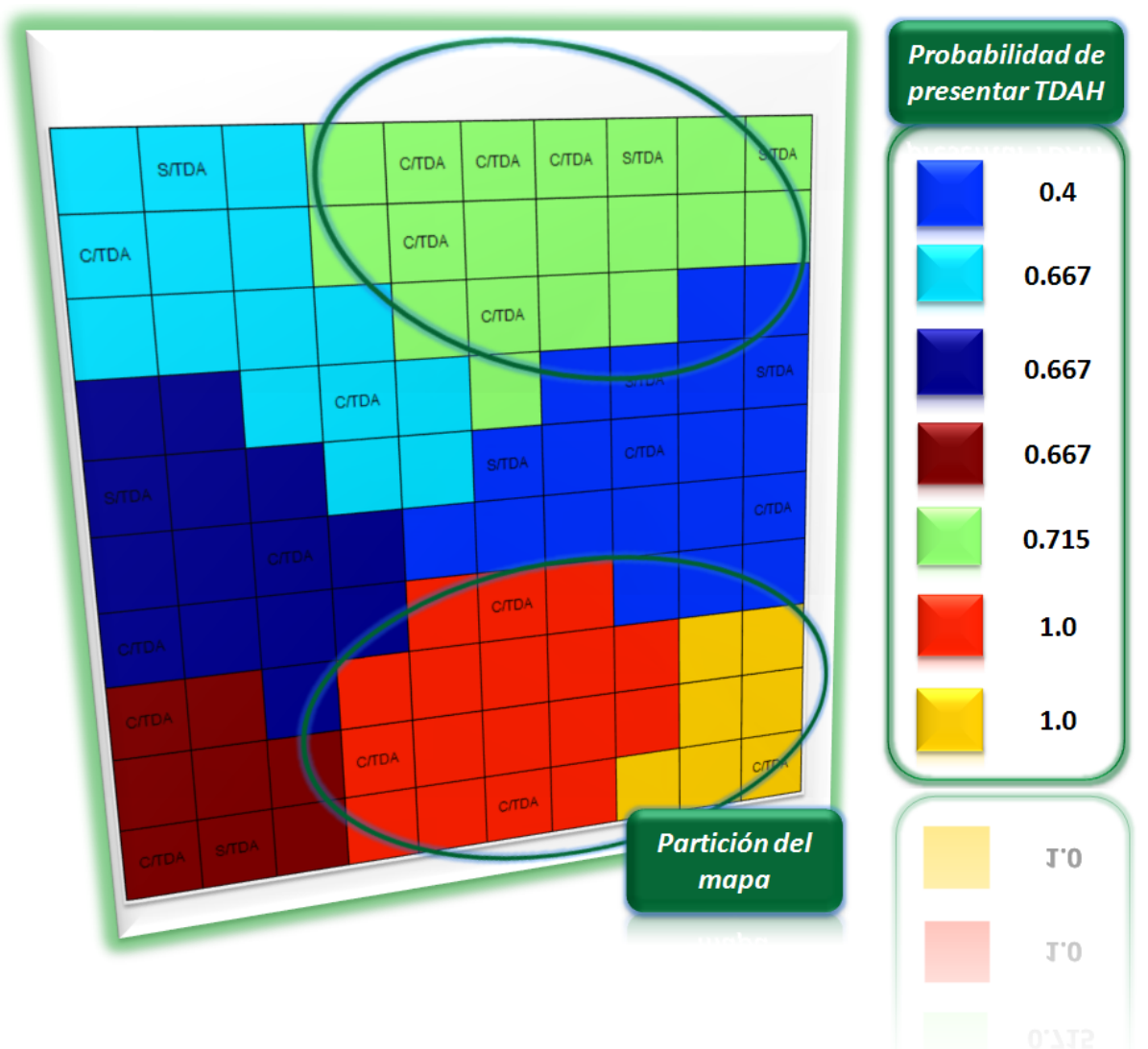

(b) Histograma de los sujetos diagnosticados en función de la región de probabilidad del mapa

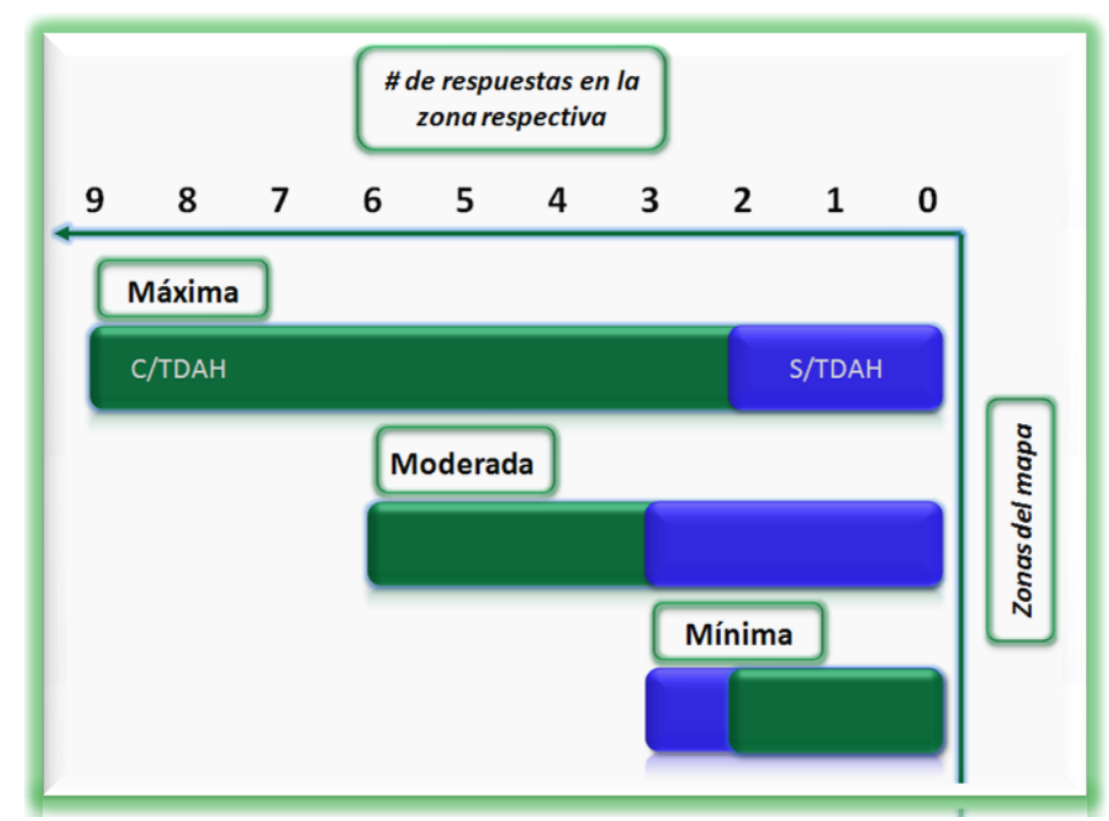

Figura 6.5: Ejemplo ilustrativo de un mapa estándar generado por la arquitectura de análisis propuesta. 


\section{Parte IV}

\section{Experimentos, Resultados y Discusión}




\section{Capítulo 7}

\section{Validación del mapa clasificador}

Aprender sin pensar es inútil.

Pensar sin aprender, es peligroso.

CONFUCIUS

Para generar los seis pares de grupos necesarios durante el proceso de validación, fue permutado aleatoriamente el número de sujetos para ambas poblaciones obteniendo así, dos vectores $v_{C / T D A}$ y $v_{s / T D A}$ a partir de los cuales fue formado tanto el conjunto de entrenamiento denotado por $s D e$ y el de validacion denotado por $s D v$ por medio de la unión entre los seis subgrupos para cada una de las vías de validación, es decir,

$$
\begin{gathered}
v_{c / T D A}=\{\underbrace{17,3,10}_{G_{1 c}}, \underbrace{7,9,12}_{G_{2 c}}, \underbrace{14,6,8}_{G_{3 c}}, \underbrace{11,2,18}_{G_{4 c}}, \underbrace{5,15,16}_{G_{5 c}}, \underbrace{4,13,1}_{G_{6 c}}\} \in \mathbb{R}^{18} \\
v_{s / T D A}=\{\underbrace{2,5}_{G_{1 s}}, \underbrace{6,7}_{G_{2 s}}, \underbrace{8,10}_{G_{3 s}, 10}, \underbrace{4,3}_{G_{4 s}}, \underbrace{9,1}_{G_{5 s}}\} \in \mathbb{R}^{10}
\end{gathered}
$$

via 1: $s D e_{1}=\left\{G_{1 c}, G_{1 s}, G_{2 c}, G_{2 s}, G_{3 c}, G_{3 s}, G_{4 c}, G_{4 s}, G_{5 c}, G_{5 s}\right\} \quad s D v_{1}=\left\{G_{6 c}, G_{6 s}\right\}$ via 2: $s D e_{2}=\left\{G_{1 c}, G_{1 s}, G_{2 c}, G_{2 s}, G_{3 c}, G_{3 s}, G_{4 c}, G_{4 s}, G_{6 c}, G_{6 s}\right\} \quad s D v_{2}=\left\{G_{5 c}, G_{5 s}\right\}$ via 3: $s D e_{3}=\left\{G_{1 c}, G_{1 s}, G_{2 c}, G_{2 s}, G_{3 c}, G_{3 s}, G_{5 c}, G_{5 s}, G_{6 c}, G_{6 s}\right\} \quad s D v_{3}=\left\{G_{4 c}, G_{4 s}\right\}$ via $4: s D e_{4}=\left\{G_{1 c}, G_{1 s}, G_{2 c}, G_{2 s}, G_{4 c}, G_{4 s}, G_{5 c}, G_{5 s}, G_{6 c}, G_{6 s}\right\} \quad s D v_{4}=\left\{G_{3 c}, G_{3 s}\right\}$ via 5: $s D e_{5}=\left\{G_{1 c}, G_{1 s}, G_{3 c}, G_{3 s}, G_{4 c}, G_{4 s}, G_{5 c}, G_{5 s}, G_{6 c}, G_{6 s}\right\} \quad s D v_{5}=\left\{G_{2 c}, G_{2 s}\right\}$ via $6: s D e_{6}=\left\{G_{2 c}, G_{2 s}, G_{3 c}, G_{3 s}, G_{4 c}, G_{4 s}, G_{5 c}, G_{5 s}, G_{6 c}, G_{6 s}\right\} \quad s D v_{6}=\left\{G_{1 c}, G_{1 s}\right\}$

Una vez definidos los sujetos que conforman a los conjuntos de entrenamiento y validación para cada una de las vías, se tomó al $s D e$ generado en cada vía de validación para someterlo a la arquitectura de análisis explicada en el capitulo anterior, consiguiendo al final de todo este proceso seis mapas entrenados, uno por cada una de las vías diseñadas.

Con todo lo anterior, la idea del funcionamiento para el soporte propuesto consiste en el proceso siguiente: cada vez que un nuevo sujeto sea presentado al mapa para ser clasificado, lo único que se tendrá que hacer es determinar la ubicación espacial sobre el mapa del nodo activado 
por este sujeto y observar a cual de los cluster encontrados pertenece, determinando con ello la región de probabilidad relacionada con dicho cluster y así, finalmente poder apoyar al experto humano clínico en su tarea diagnóstica, mostrandole por medio de una codificación en color, en que región de probabilidad de presentar TDAH se encuentra dicho sujeto.

\subsection{Resultados del proceso de validación cruzada}

La tabla 7.1 resume los resultados encontrados, para todas las vías de validación, después de aplicar los puntos mencionados en líneas anteriores respecto al procesamiento de los mapas entrenados tanto para los rasgos frecueciales como para los wavelets.

Tabla 7.1: Cifras relevantes arrojadas por el proceso de valización cruzada.

\begin{tabular}{|c|c|c|c|c|}
\hline Validación & $\begin{array}{c}\text { Rasgos Frecuenciales: } \\
\text { Nodos activados }\end{array}$ & Tasa de acierto $t_{a}$ & $\begin{array}{c}\text { Rasgos Wavelet: } \\
\text { Nodos activados }\end{array}$ & Tasa de acierto $t_{a}$ \\
\hline vía 1 & $\{50,50,98,91,98\}$ & $\frac{4}{5}=0,8$ & $\{92,51,98,51,51\}$ & $\frac{4}{5}=0,8$ \\
vía 2 & $\{25,31,20,20,20\}$ & $\frac{4}{5}=0,8$ & $\{91,73,51,83,91\}$ & $\frac{3}{5}=0,6$ \\
vía 3 & $\{91,91,91,71,91\}$ & $\frac{3}{5}=0,6$ & $\{42,42,42,81,42\}$ & $\frac{4}{5}=0,8$ \\
vía 4 & $\{80,80,80,10,80\}$ & $\frac{4}{5}=0,8$ & $\{41,91,61,61,91\}$ & $\frac{3}{5}=0,6$ \\
vía 5 & $\{60,95,41,95,90\}$ & $\frac{3}{5}=0,6$ & $\{99,59,98,98,10\}$ & $\frac{4}{5}=0,8$ \\
vía 6 & $\{10,10,46,46,3\}$ & $\frac{3}{5}=0,6$ & $\{15,15,15,51,51\}$ & $\frac{5}{5}=1,0$ \\
& & & & \\
\hline$t_{a}$ & & 0.7 & & 0.7667 \\
$s_{t_{a}}$ & & 0.1095 & & 0.1506 \\
\hline
\end{tabular}

La segunda y cuarta columna de la tabla 7.1 se refieren al conjunto de nodos que resultaron ganadores, es decir, que fueron activados al presentarle al mapa su correspondiente conjunto de validación mientras que la tercera y quinta columna, indican el cociente entre el número de veces que el mapa clasificó correctamente al sujeto basado en la región de probabilidad donde se encontrara el nodo activado y el total, tanto para los rasgos frecuenciales como para los rasgos wavelet respectivamente.

Por otro lado, los parámetros $\overline{t_{a}}$ y $s_{t_{a}}$ se refieren al valor de rendimiento medio del mapa clasificador asi como a su desviación estándar respectivamente, pudiendo concluir de ellos que la eficiencia del soporte se moverá aproximadamente de un $60 \%$ a un $80 \%$ respecto a su capacidad acertada de clasificación.

Por otra parte, las gráficas generadas por los cuatro procesos son las mostradas en la figura 7.1 para la validación en la primera y segunda vía, la figura 7.2 para la tercera y cuarta vía y la figura 7.3 para la quinta y sexta vía de validación, respecto únicamente a los rasgos frecuenciales. Para las últimas figuras, en cada vía, correspondientes al proceso de clustering se marcaron con círculos grises sobre el mapa final, los nodos activados por el conjunto de validación respectivo y por último, para los rasgos wavelet los mapas finales generados en cada una de las vías son los mostrados en la figura 7.4. 
Vía 1: $t_{a}=\frac{4}{5}$

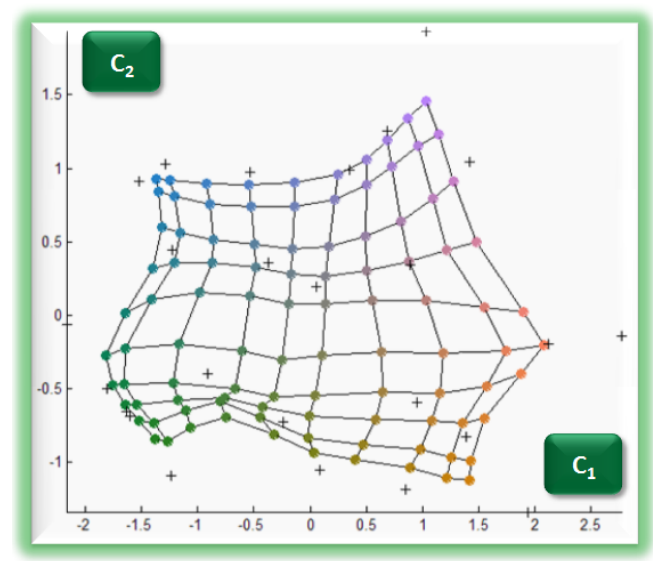

Proceso 1: PCA

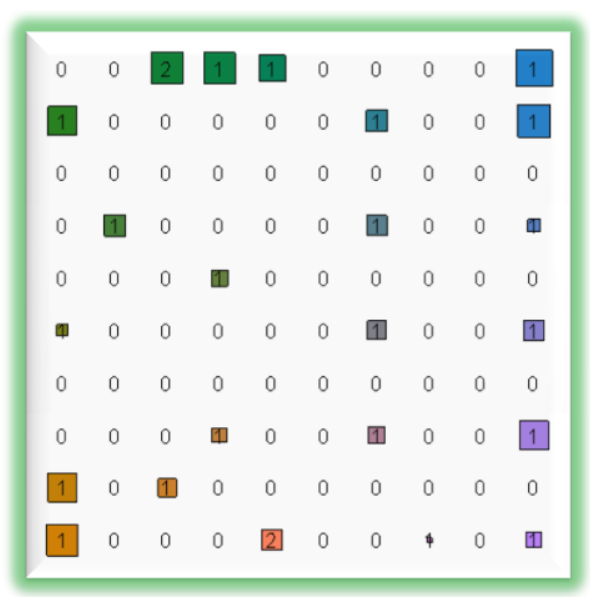

\section{Proceso 2: Histograma}

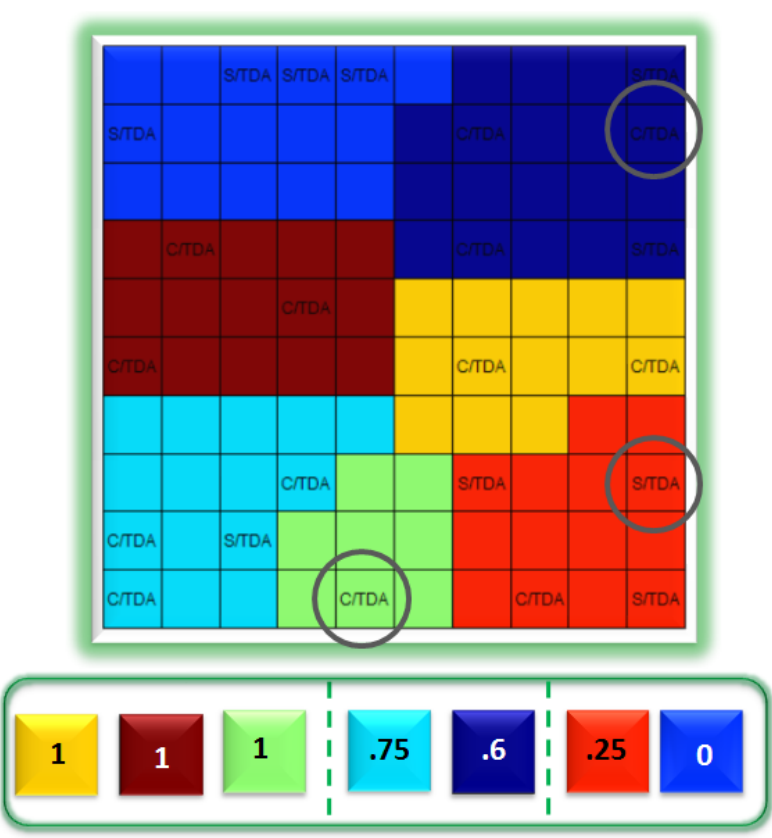

Proceso 3 y 4: Clustering y Región de probabilidad
Vía 2: $t_{a}=\frac{4}{5}$

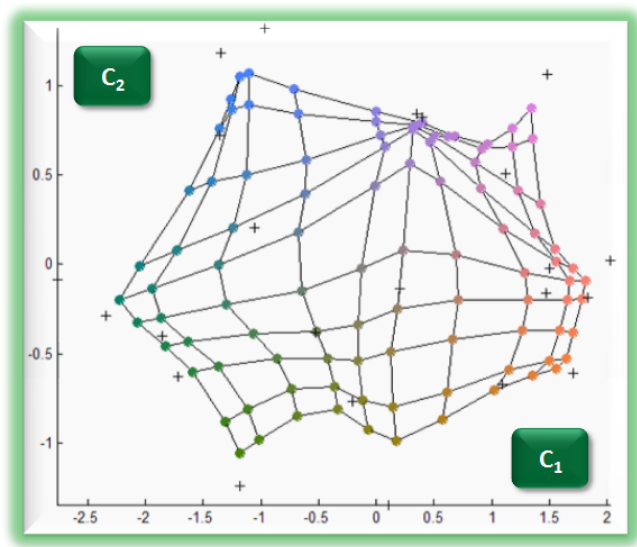

Proceso 1: PCA

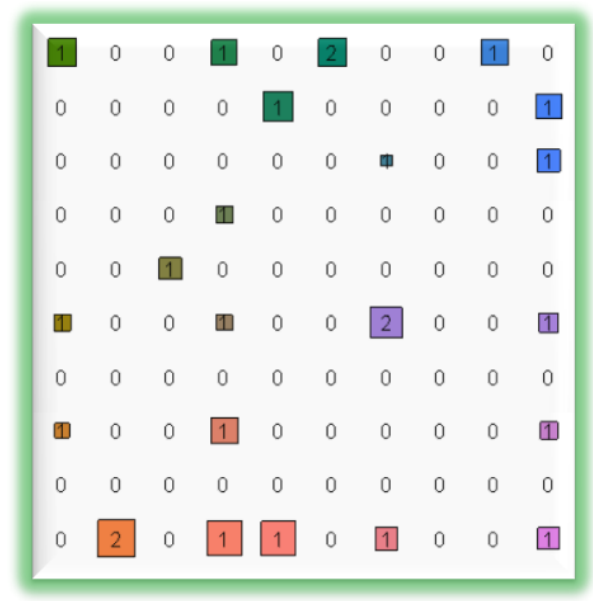

Proceso 2: Histograma

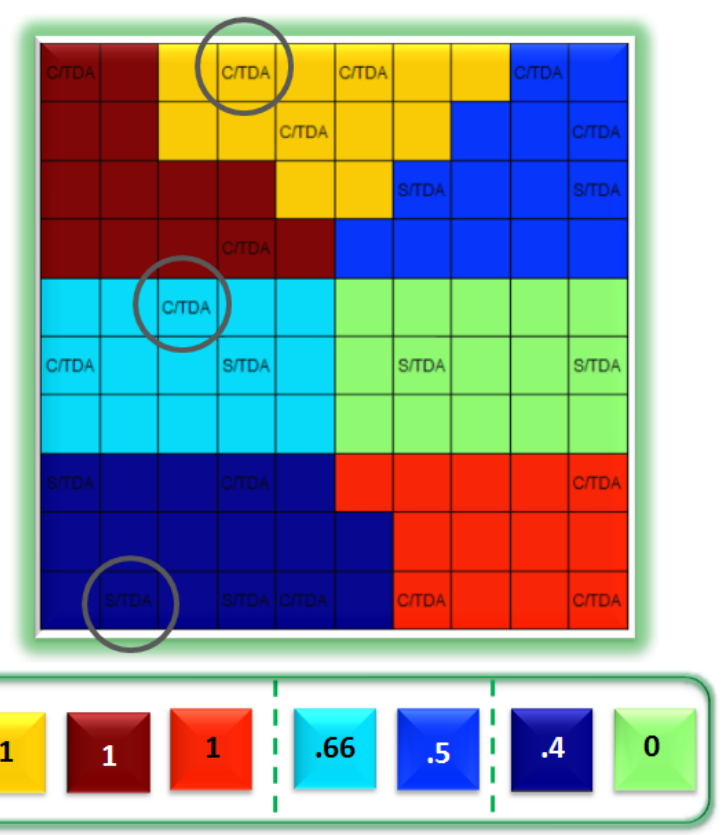

Proceso 3 y 4: Clustering y Región de probabilidad Figura 7.1: Graficas generadas para las dos primeras vías de validación. 
Vía 3: $t_{a}=\frac{3}{5}$

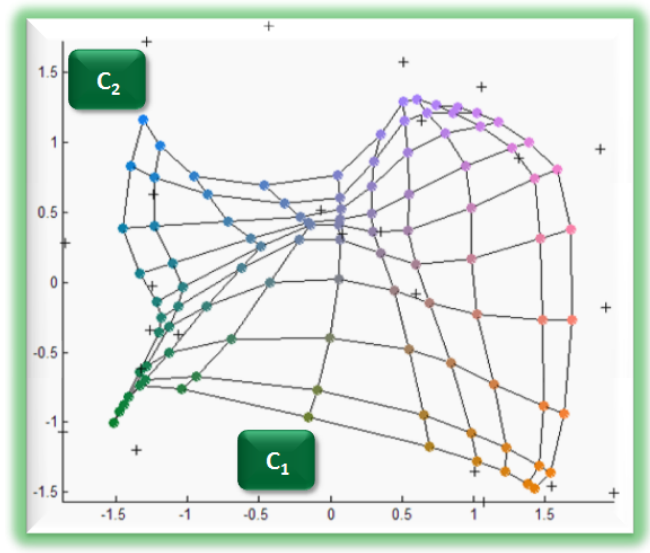

Proceso 1: PCA

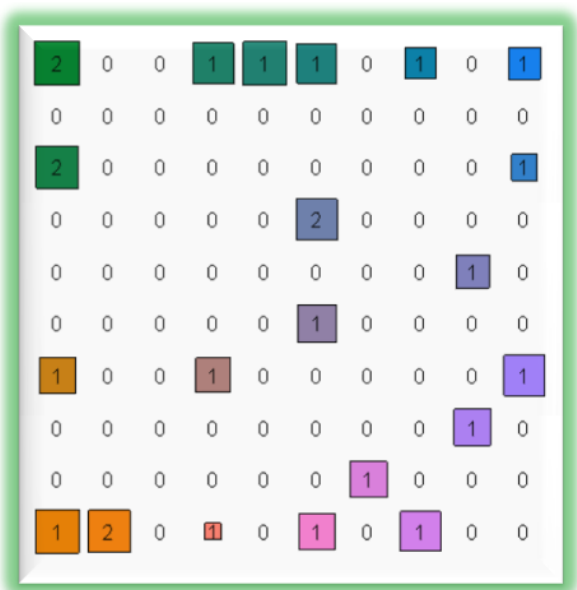

Proceso 2: Histograma

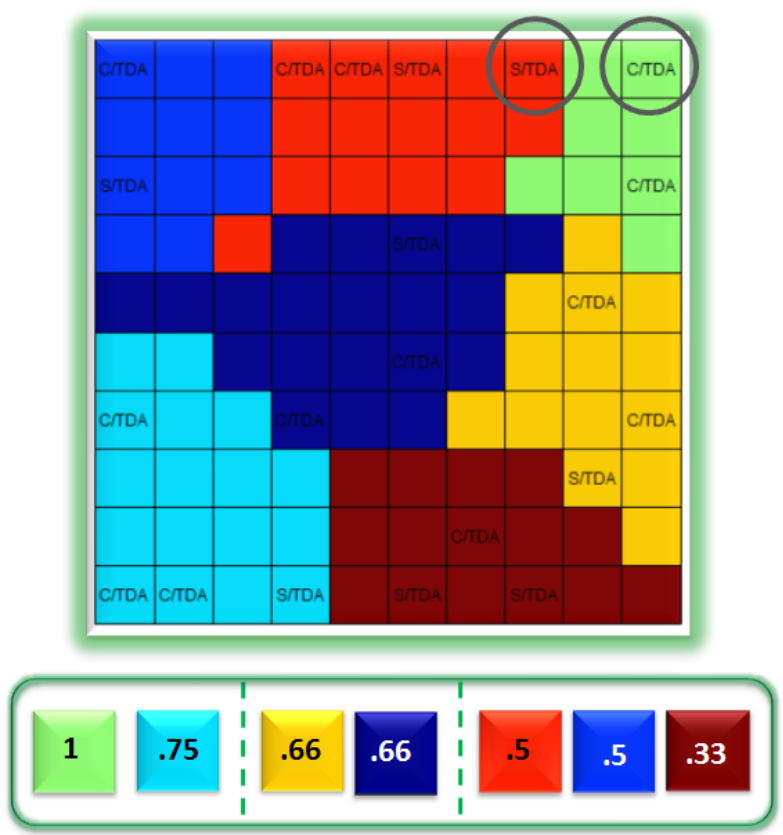

Proceso 3 y 4: Clustering y Región de probabilidad
Vía 4: $t_{a}=\frac{4}{5}$

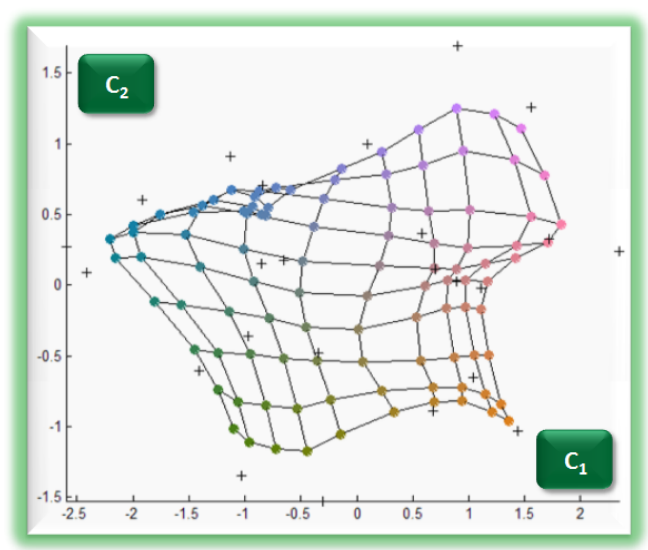

Proceso 1: PCA

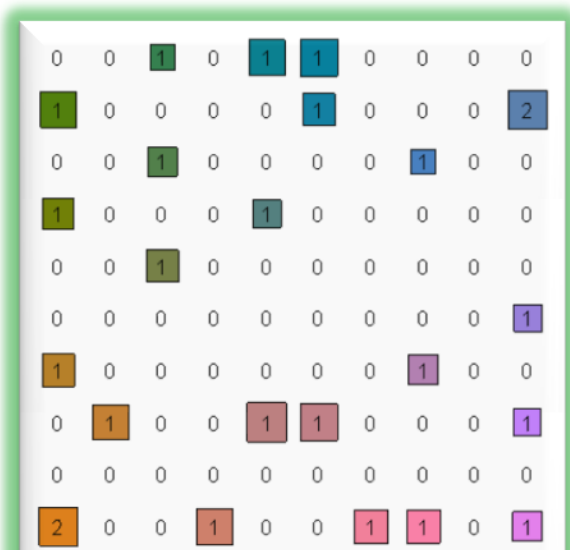

Proceso 2: Histograma

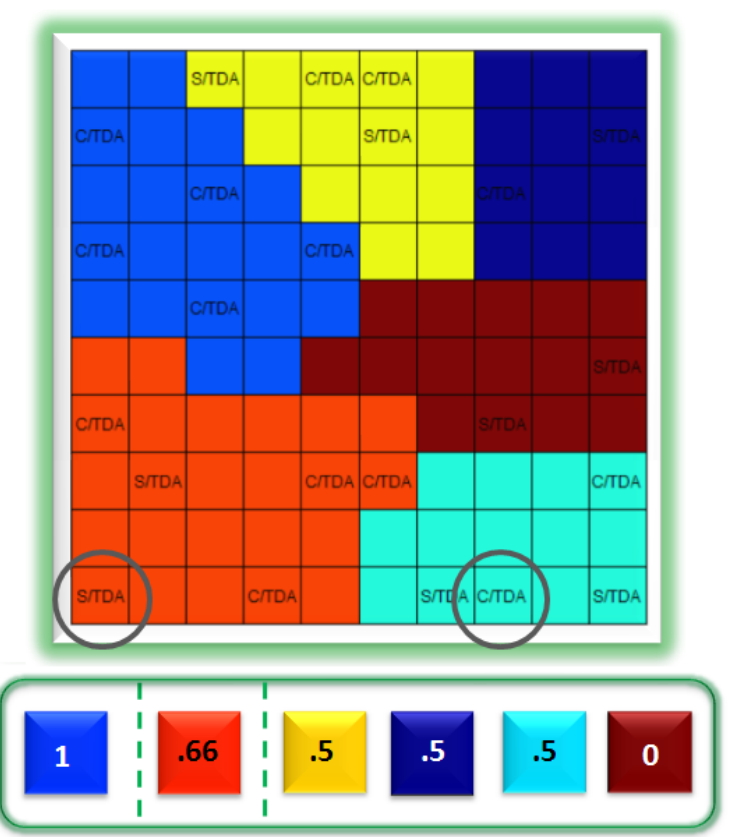

Proceso 3 y 4: Clustering y Región de probabilidad

Figura 7.2: Graficas generadas para las dos segundas vías de validación. 
Vía 5: $t_{a}=\frac{3}{5}$

Vía 6: $t_{a}=\frac{3}{5}$

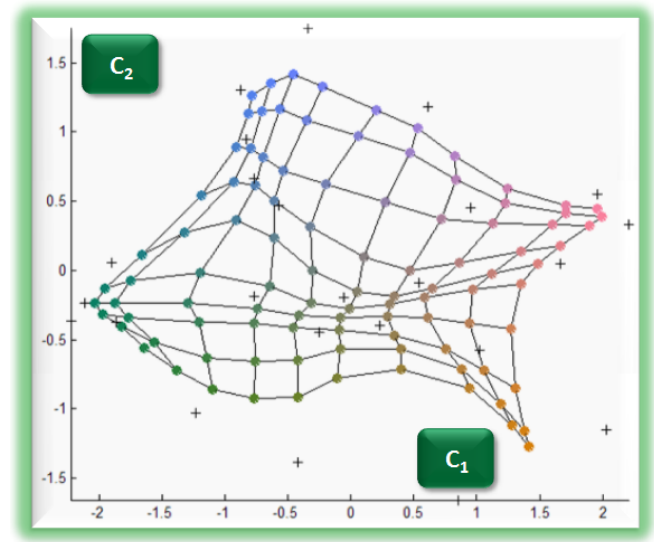

Proceso 1: PCA

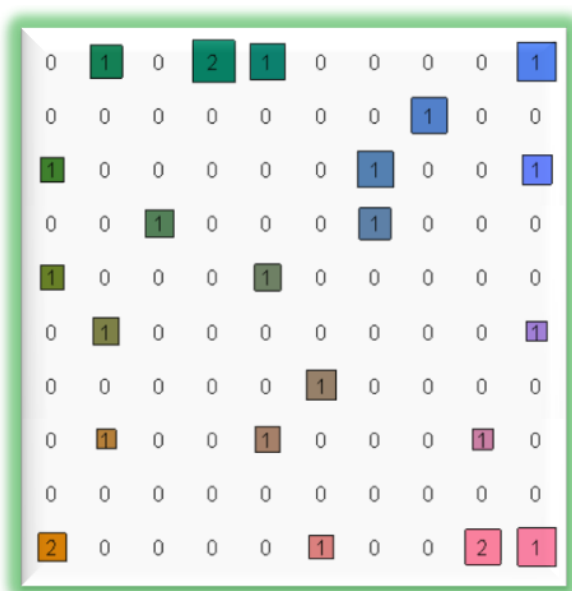

Proceso 2: Histograma

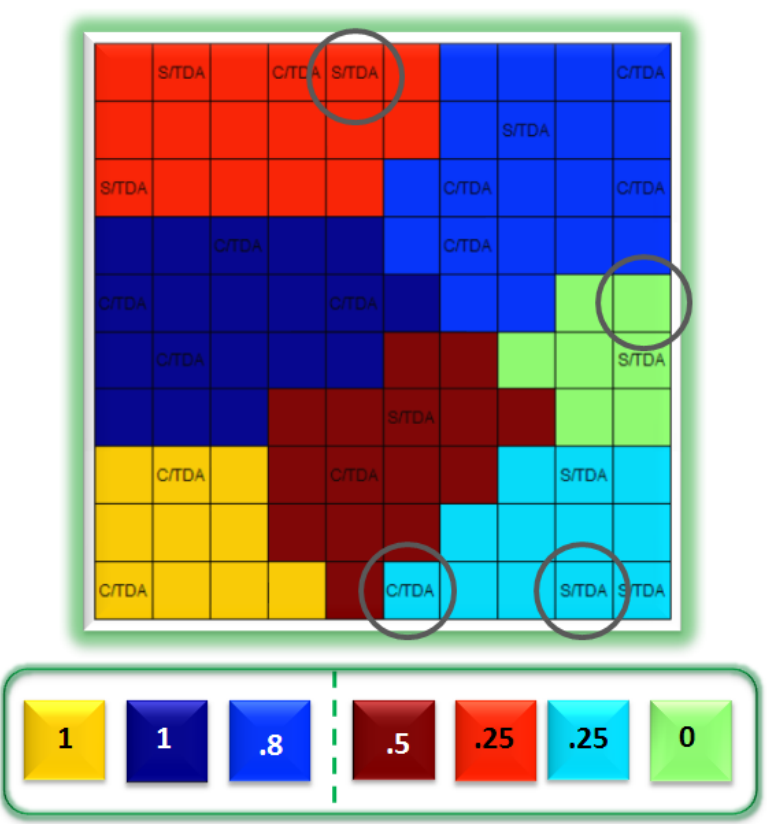

Proceso 3 y 4: Clustering y Región de probabilidad

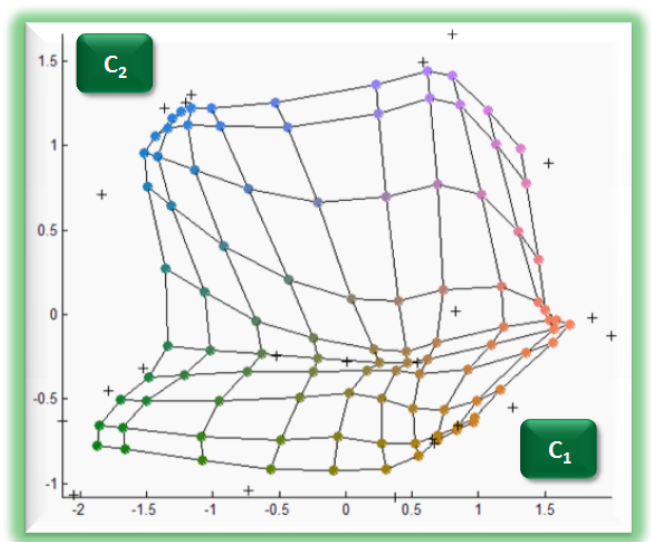

Proceso 1: PCA

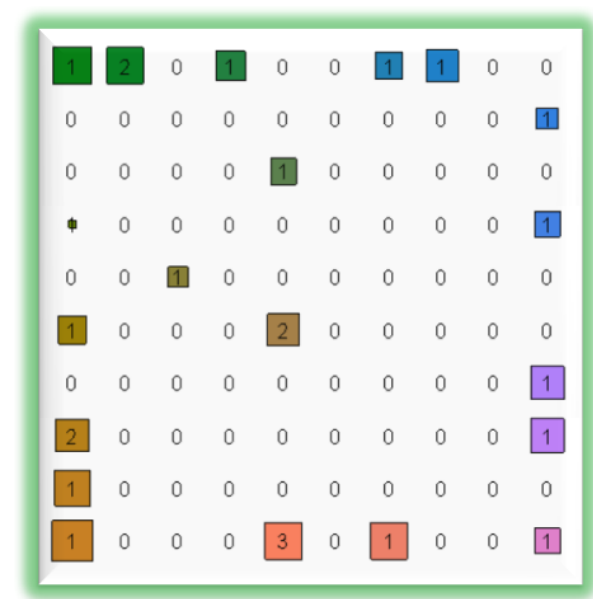

Proceso 2: Histograma

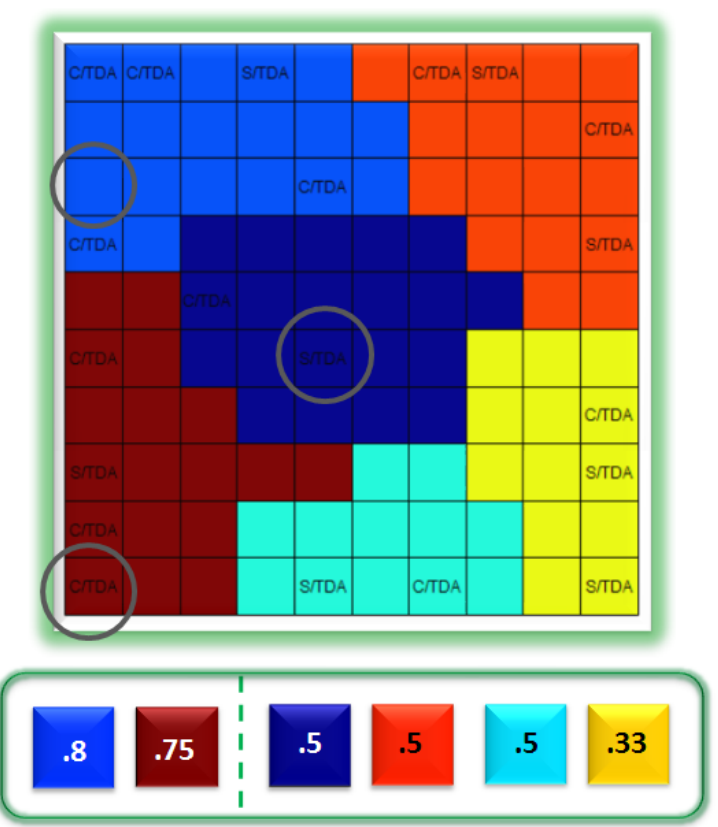

Proceso 3 y 4: Clustering y Región de probabilidad

Figura 7.3: Graficas generadas para las dos terceras vías de validación. 
Figura 7.5: Mapas clasificadores resultantes del proceso de validación respecto a los rasgos wavelet extraídos.

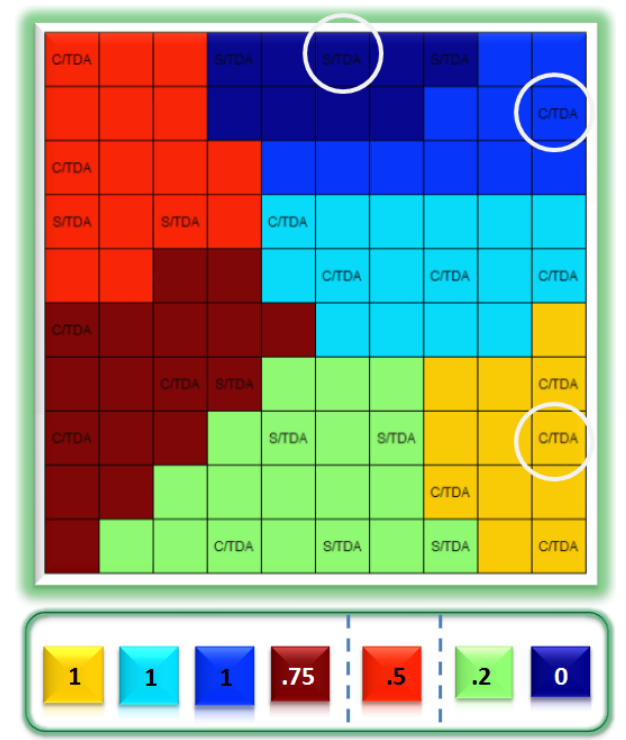

Vía 1: $t_{a}=\frac{4}{5}$

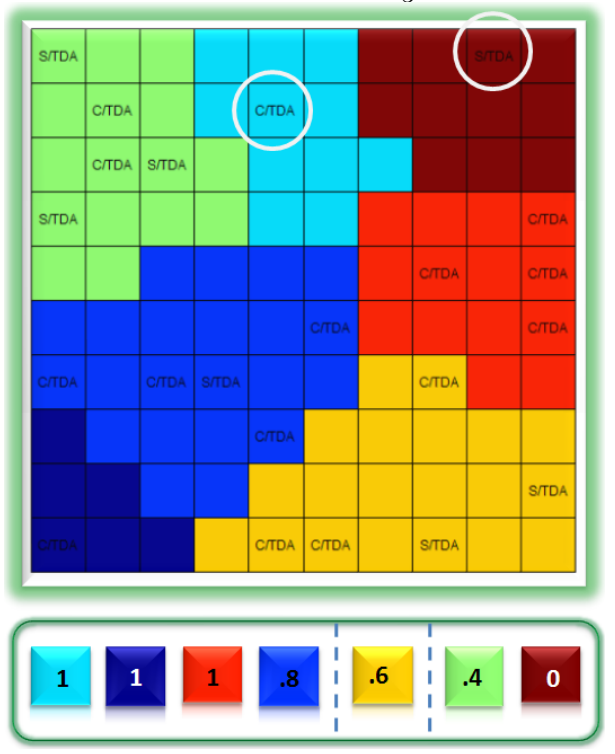

Vía 3: $t_{a}=\frac{4}{5}$
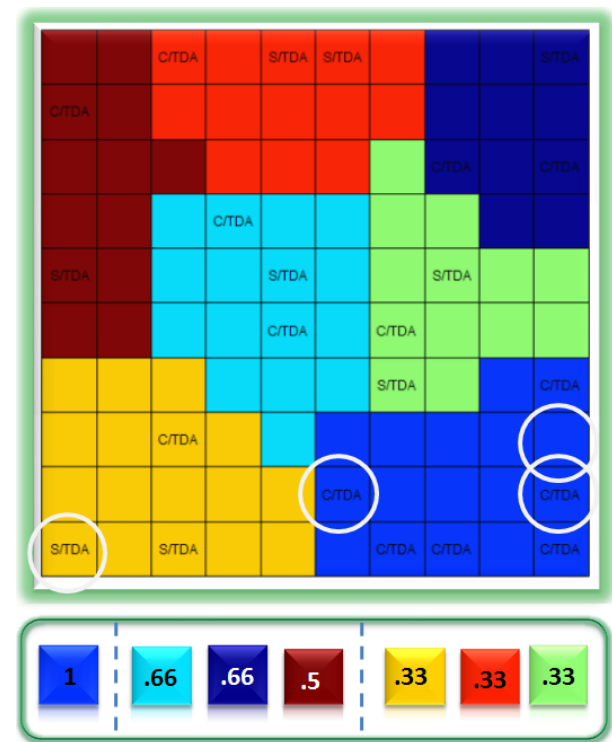

Vía $5: t_{a}=\frac{4}{5}$
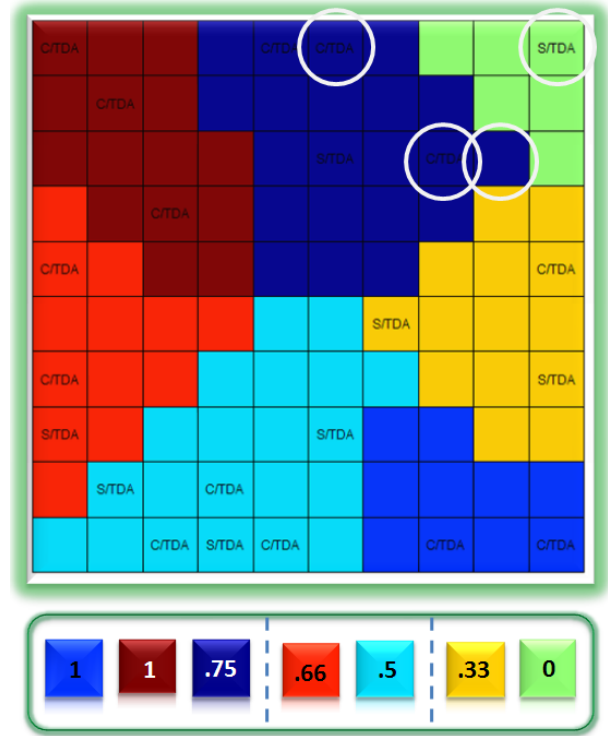

Vía 2: $t_{a}=\frac{3}{5}$
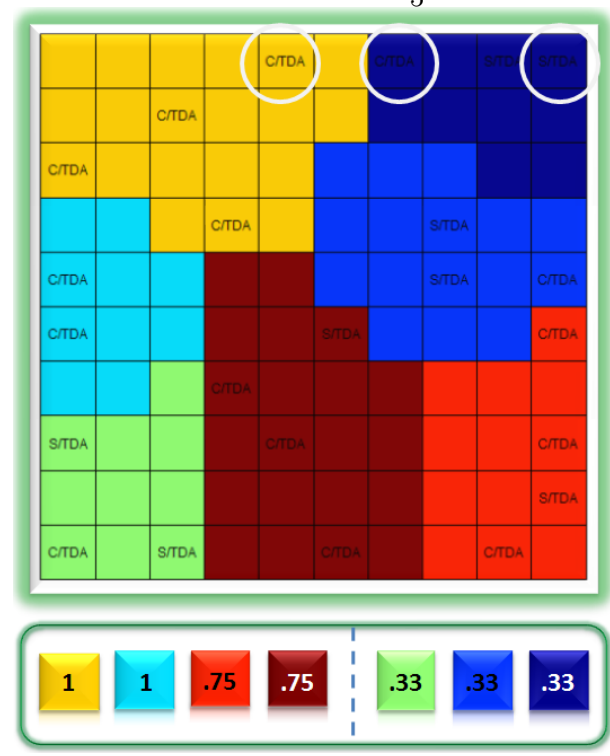

Vía $4: t_{a}=\frac{3}{5}$
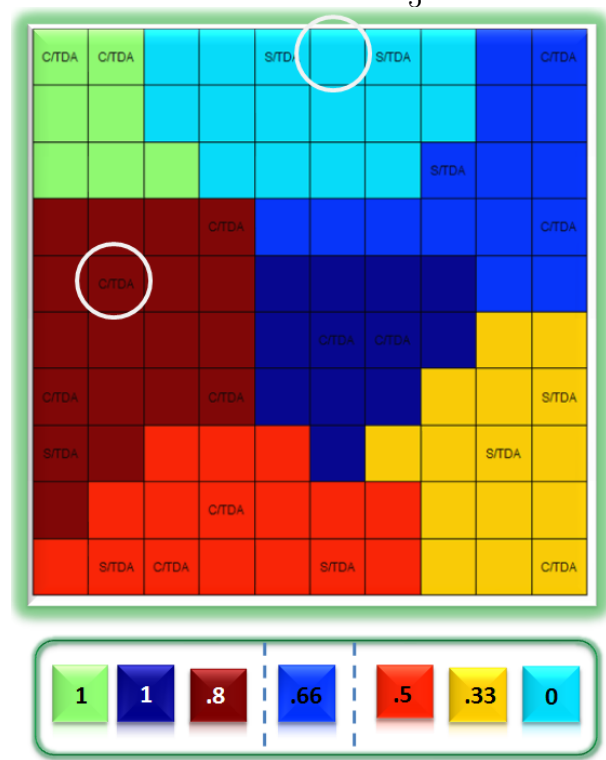

Vía 6: $t_{a}=1$ 


\section{Capítulo 8}

\section{Sistema de detección $S O M_{f}$ para el soporte al diagnóstico de TDAH}

Enseña a tus alumnos cómo pensar, no qué pensar. SIDNEY SUGARMAN

En este capítulo se muestra la evolución en la implementación del sistema de soporte al diagnóstico de TDAH propuesto, presentado para ello todos los mapas generados en cada uno de los niveles de análisis, unicamente, respecto al primer método utilizado para la extracción de características (rasgos frecuenciales). Básicamente fue propuesto un prototipo final para dicho sistema de soporte, por supuesto limitado al tamaño de la población (28 niños en total) y abordando también de manera más minuciosa todos los aspectos relacionados con la interpretación respecto a la capacidad de apoyo del sistema durante la tarea diagnóstica así como a la información brindada por el mismo.

\subsection{Mapas SOM multicapa como clasificadores}

Una vez realizado el proceso de validación, se generó el prototipo final para el mapa clasificador llevando a cabo el mismo procesamiento y análisis de éste, mencionado en líneas anteriores, pero en esta ocasión fueron tomados de manera aleatoria veintidos sujetos de la pobación de estudio, catorce de los cuales diagnosticados con TDAH y ocho sin TDAH, para con ello formar al conjunto de entrenamiento mientras que para el conjunto de validación fueron tomados a los sujetos restantes constituidos de cuatro con TDAH y dos sin TDAH, ésto con dos fines principales:

1. Primeramente, se buscó observar el comportamiento del sistema de soporte brindado durante cada uno de los niveles de análisis, es decir, apreciar la forma en que trabajan tanto los mapas entrenados por cada ritmo cerebral generados en el primer nivel de analisis como el mapa clasificador final construido durante el segundo nivel de análisis a partir de la combinación en información de los primeros mapas generados.

2. Por otro lado, también resultó sumamente interesante poder determinar, para cada uno de los sujetos del grupo de validación, si la clasificación realizada en estos dos niveles de análisis resultó coherente, lo cual se refiere a si en efecto para cada uno de los niveles un cierto sujeto fue clasificado dentro del mismo rubro ya sea con TDAH o bien sin TDAH. 


\subsubsection{Capa I: Mapas por onda cerebral $S O M_{\theta}, S O M_{\alpha}$ y $S O M_{\beta}$}

Los mapas generados en este primer nivel de análisis, fueron formados de manera independiente mediante la búsqueda de correlaciones inmersas en cada uno de los ritmos cerebrales estudiados. Desafortunadamente, debido a la gran cantidad de ruido de baja frecuencia (producido por movimientos o artefactos) inmerso en las señales cerebrales durante la adquisición de los registros, al encontrar la mayoría de la potencia media de dichas señales en las frecuencias del ritmo delta, se presentó la necesidad de descartar la idea de generar el mapa para este ritmo.

A continuación se presentan los mapas generados en la primera capa, rescatando de ellos, primero, los clusters encontrados durante el proceso; segundo, las regiones de probabilidad definidas de presentar TDAH y sobre todo la clasificación resultante para los sujetos realizada por estos mapas.

\section{Mapa Theta: $S O M_{\theta}$}

En la figura 8.1 se muestra el mapa obtenido para el ritmo Theta, en el cual podemos apreciar siete clusters que definen tres regiones de probabilidad; máxima (clusters azul turquesa y verde), moderada (cluster guinda) y mínima (clusters azul claro, marino, naranja y amarillo).

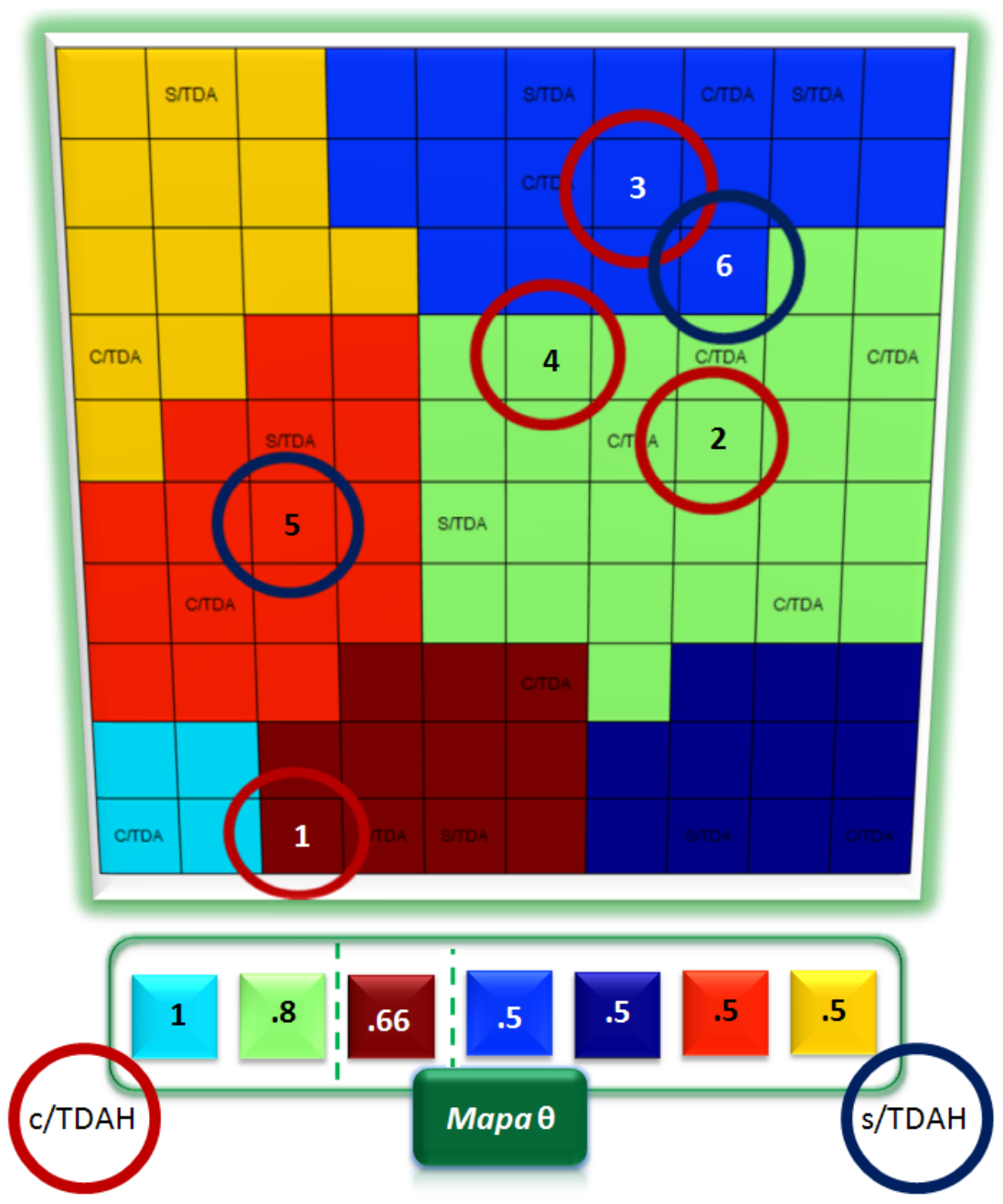

Figura 8.1:

Mapa clasificador etiquetado, con clusters encontrados (codificados en color) y regiones de probabilidad definidas (indicadas en el renglón de cuadros en la parte inferior del mapa), para el ritmo $\theta$ desarrollado en el nivel uno de la arquitectura de análisis. 
Mapa Alpha: $S O M_{\alpha}$

Por otro lado, en la figura 8.2 es mostrado el mapa obtenido para el ritmo Alpha, el cual al igual que el anterior se compuso de siete clusters que definieron de igual manera tres regiones de probabilidad; máxima (clusters azul claro y turquesa), moderada (cluster naranja, azul marino y amarillo) y mínima (clusters guinda y verde).

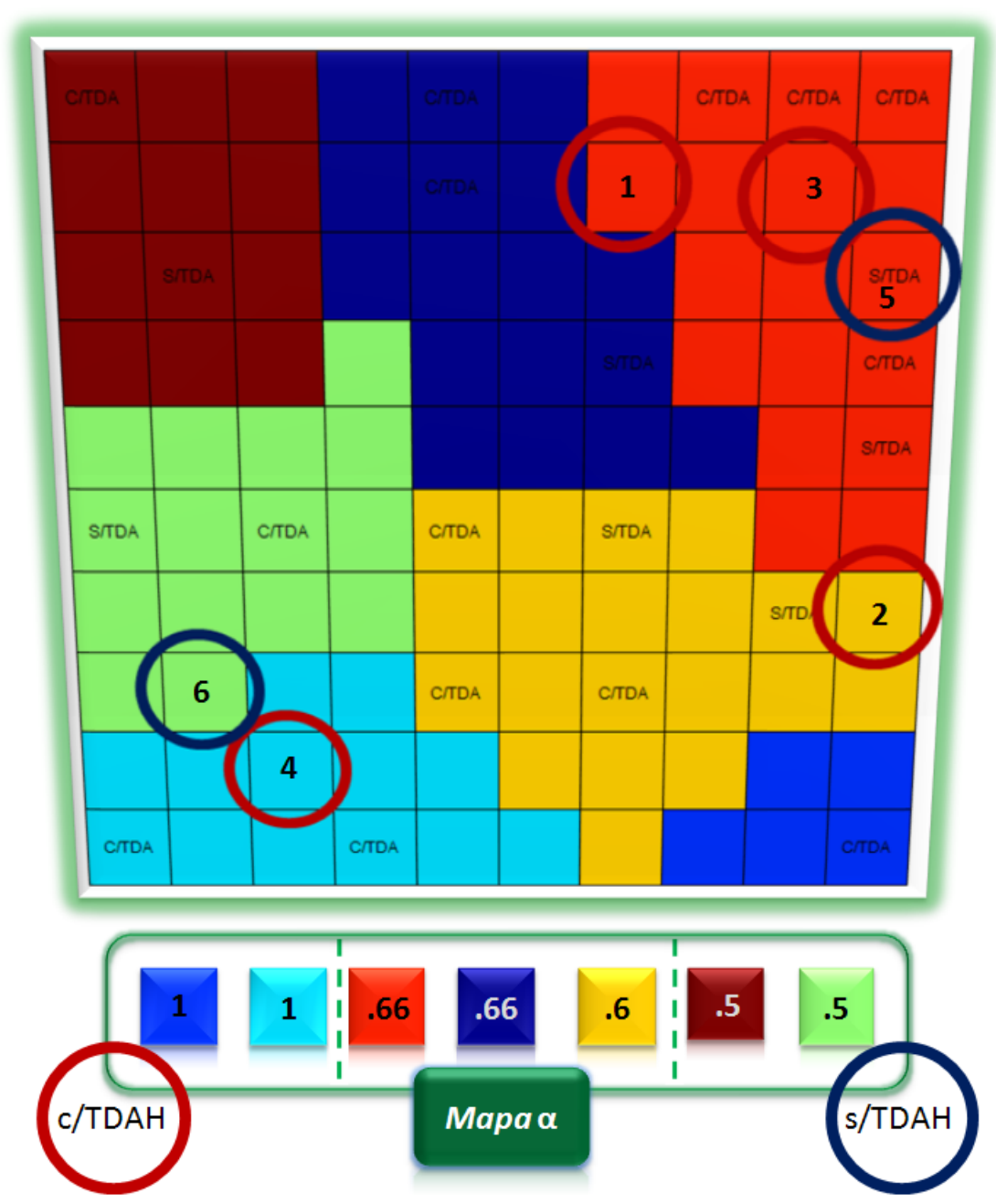

Figura 8.2:

Mapa clasificador etiquetado, con clusters encontrados (codificados en color) y regiones de probabilidad definidas (indicadas en el renglón de cuadros en la parte inferior del mapa), para el ritmo $\alpha$ desarrollado en el nivel uno de la arquitectura de análisis.

Mapa Beta: $S O M_{\beta}$

Finalmente, en la figura 8.3 se muestra el mapa obtenido para el ritmo Beta, donde fueron encontrados en esta ocasión seis clusters cuyas tres regiones de probabilidad fueron definidas de la forma siguiente: máxima (clusters azul claro y marino), moderada (cluster guinda) y mínima (clusters amarillo, naranja y azul turquesa).

Respecto a la clasificación efectuada por cada uno de los mapas, éstos trabajaron según los resultados mostrados en la tabla 8.1 de la cual se puede apreciar, que sólo en un caso que debería haber sido positivo se encontró un valor de probabilidad medio menor a 0,7 (sujeto tres) al igual que para un caso negativo (sujeto 5 ) el valor de probabilidad medio fue superior a 0,5 observando 
además que para ambos casos, el comportamiento de los tres mapas fue similar.

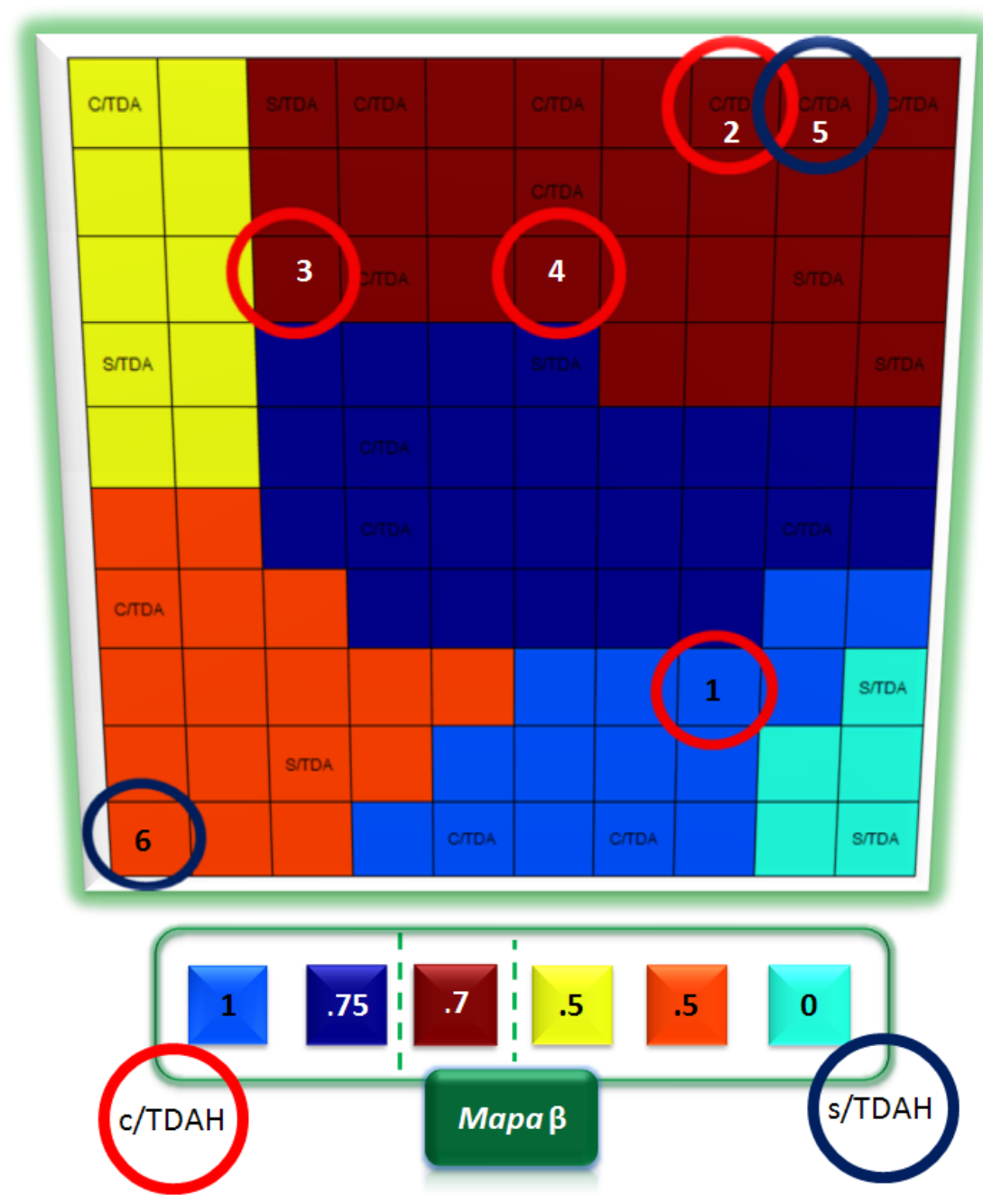

Figura 8.3:

Mapa clasificador etiquetado, con clusters encontrados (codificados en color) y regiones de probabilidad definidas (indicadas en el renglón de cuadros en la parte inferior del mapa), para el ritmo $\beta$ desarrollado en el nivel uno de la arquitectura de análisis.

Tabla 8.1:

Comparación de los resultados en la clasificación de los sujetos de validación para cada uno de los mapas generados en la primera capa del sistema propuesto.

\begin{tabular}{|c|c|c|c|c|c|}
\hline Sujeto & $P\left(S O M_{\theta}\right)$ & $P\left(S O M_{\alpha}\right)$ & $P\left(S O M_{\beta}\right)$ & Valor medio & Diagnóstico maestro \\
\hline & & & & & \\
1 & 0.66 & 0.66 & 1.0 & 0.77 & c/TDAH \\
2 & 0.8 & 0.6 & 0.7 & 0.7 & c/TDAH \\
3 & 0.5 & 0.66 & 0.7 & 0.62 & c/TDAH \\
4 & 0.8 & 1.0 & 0.7 & 0.83 & c/TDAH \\
5 & 0.5 & 0.66 & 0.7 & 0.62 & s/TDAH \\
6 & 0.5 & 0.5 & 0.5 & 0.5 & s/TDAH \\
& & & & & \\
\hline
\end{tabular}




\subsubsection{Capa II: Combinación en información de ritmos cerebrales}

El objetivo de esta segunda capa, consistió en generar un único mapa clasificador, con características iguales a los mapas anteriores, mediante un nuevo aprendizaje de las correlaciones encontradas durante el primer entrenamiento es por ello que este nuevo mapa fue formado primero, por la combinación en información de los ritmos cerebrales estudiados y segundo, por la combinación en información de las señales bipolares registradas.

$$
\left\{S O M_{\theta}, S O M_{\alpha}, S O M_{\beta}\right\} \rightarrow S O M_{f}
$$

A continuación se muestra en la figura 8.4, la propuesta del prototipo final para el mapa clasificador generado, con la metodología de análisis propuesta, por los rasgos frecuenciales mediante el estudio clásico de las fluctuaciones en la potencia espectral para las distintas regiones espaciales donde fue aplicada la fotoestimulación.

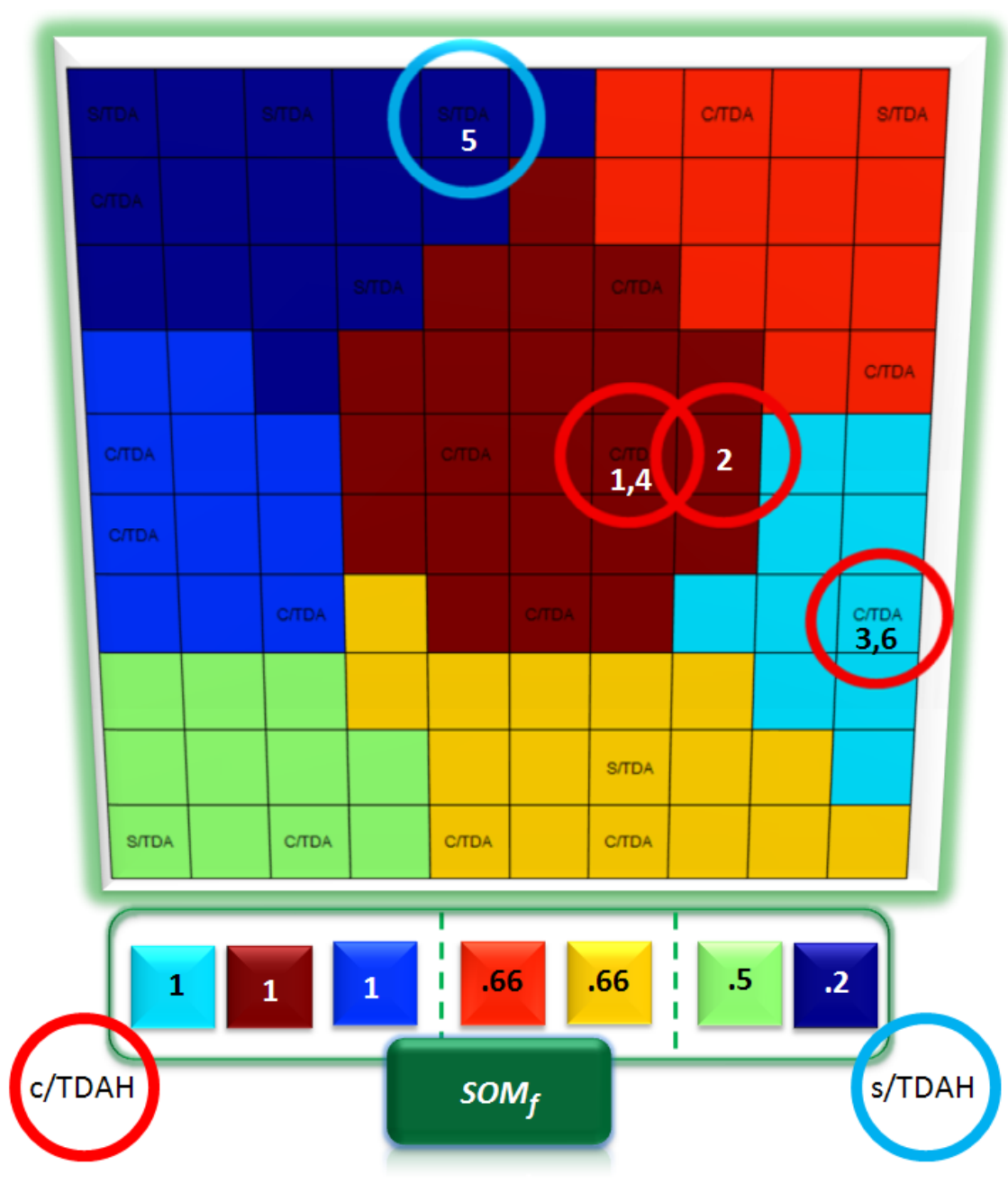

Figura 8.4:

Prototipo final para el mapa clasificador $S O M_{f}$ generado por los rasgos frecuenciales en el nivel dos de la arquitectura de análisis, mostrando las mismas características de los maspas del primer nivel.

En este último mapa de la figura 8.4, se pudo observar que la eficiencia en la clasificación mejoró respecto al desempeño de los mapas del nivel anterior ya que el sistema, en la totalidad de los casos positivos, no solo clasificó de manera acertada sino que los localizó en regiones de probabilidad máxima algo que no sucedió con los otros mapas además de sólo equivocarse únicamente en uno de los casos negativos (sujeto 6). 
$\left\{S O M_{F_{p 1}-F_{3}}, S O M_{F_{3}-C_{3}}, \cdots, S O M_{P_{4}-O_{2}}\right\} \rightarrow S O M_{f}$

Ahora bien, al basarse en un método contemporáneo como lo es el análisis multiresolución se pudo generar también un prototipo de mapa clasificador final cuyo desempeño es el mostrado en la figura 8.5, en el cual se puede apreciar que la clasificación realizada por éste resultó concisa, respecto al diagnóstico maestro, para todos los casos presentados; los sujetos 1, 2 y 4 cayeron en nodos con etiqueta positiva de la misma forma, el sujeto 5 cayó en nodo con etiqueta negativa mientras que para los sujetos 3 y 6 la región de probabilidad determinó su condición alta y baja respectivamente.

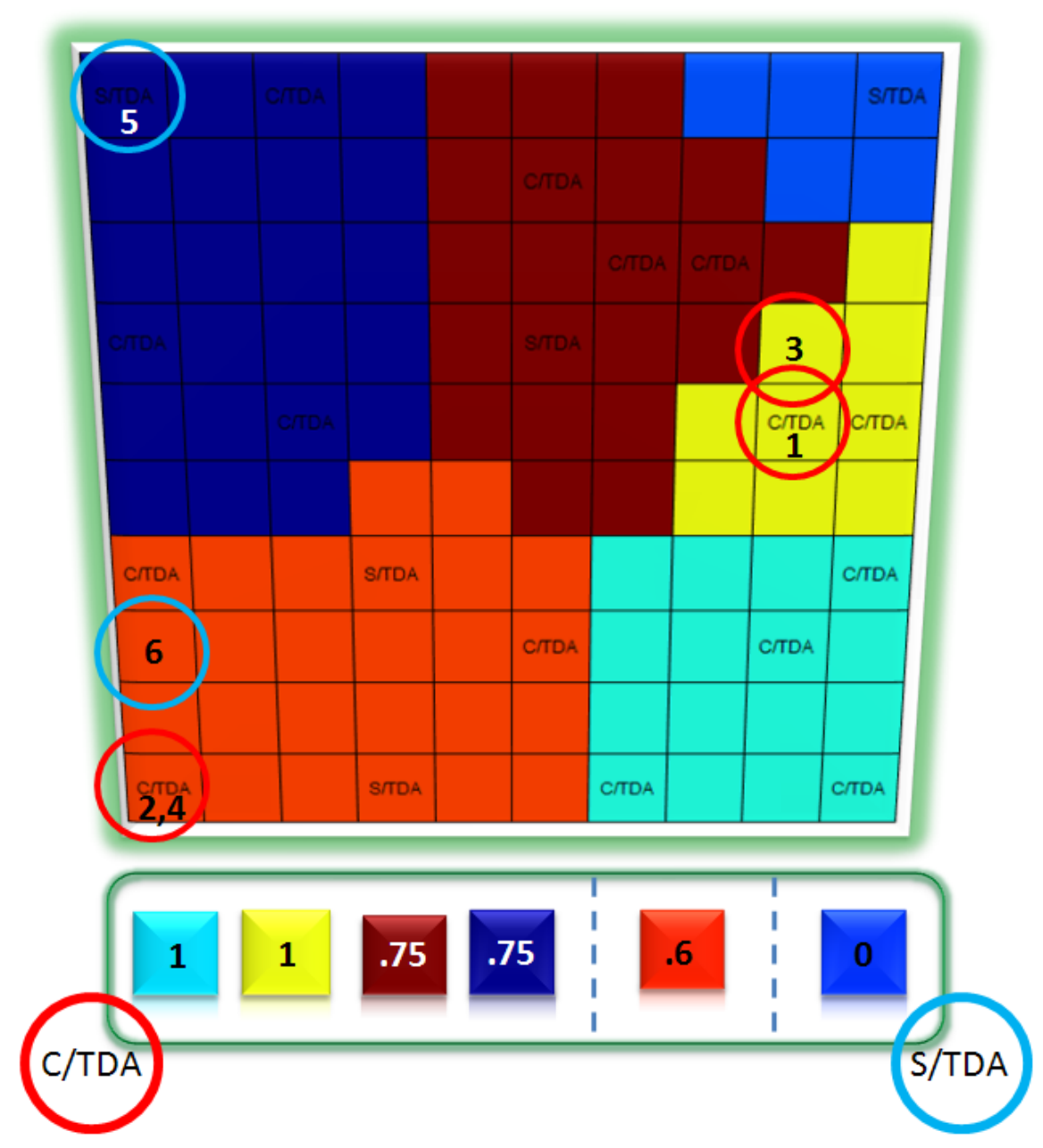

Figura 8.5:

Prototipo final para el mapa clasificador $S O M_{f}$ generado por los rasgos wavelet en el nivel dos de la arquitectura de análisis, mostrando las mismas características de los maspas del primer nivel. 


\section{Capítulo 9}

\section{Discusión y Conclusiones}

La inteligencia consiste no sólo en el conocimiento, sino también en la destreza de aplicar los conocimientos en la práctica.

ARISTÓTELES

\subsection{Discusión}

Se han desarrollado diversos estudios con el objeto de encontrar una herramienta que contribuya a enriquecer el diagnóstico basado en evidencia. En el caso de la Imagenología, se han obtenido imágenes con SPECT, se refería baja actividad neuronal en sujetos con TDAH [21], sin embargo, aún es controversial la causa de tal disminución del flujo sanguíneo en dicha región cerebral.

Por su parte, las investigaciones que han empleado imágenes de PET en personas con TDAH [22], no han sido concluyentes en encontrar diferencias entre los sujetos control y los que padecen el desorden debido a que no se tienen resultados reproducibles y a que no se ha podido demostrar que la causa de los aparentes cambios sea el TDAH [23].

En cuanto a la electroencefalografía, pocos trabajos de investigación han propuesto métodos cuantitativos de soporte al diagnóstico de desórdenes mentales tales como lo es el TDAH, fuera del estudio clásico del comportamiento en las fluctuaciones de la potencia espectral del EEG en niños con y sin presencia de la patología [1-5], por ende el subsecuente avance a nivel clínico de esta condición sigue basado en enfoques puramente cualitativos. Es por ello, que resulta muy importante presentar propuestas de posibles enfoques cuantitativos como la desarrollada durante este proyecto de investigación.

A diferencia de las investigaciones antes citadas [21-23], este estudio considera grupos de poblaciones y por lo tanto no compara a los sujetos, por el contrario los sujetos dadas sus características cerebrales (ambos conjuntos de rasgos frecuenciales y wavelet extraidos en este trabajo) se diferencían entre si, sin importar la edad y como consecuencia sin ser relevante el tamaño del cerebro del sujeto. 
Es de relevancia observar que la información que arroja el mapa clasificador final $S O M_{f}$ no depende de la colaboración del sujeto a estudiar ni tampoco de terceras personas, lo cual elimina gran parte del sesgo inmerso en el diagnóstico, además de que disminuye el efecto de la edad y las características antropométricas del sujeto sometido a un diagnóstico de este tipo.

La herramienta diseñada en esta investigación ofrece una atractiva opción para contribuir al diagnóstico de la patología en cuestión, teniendo siempre en mente que la eficiencia de clasificación de los mapas auto-organizados es proporcional al número de datos con que sean entrenados, es decir, que al incrementar la cantidad de datos durante el proceso de entrenamiento, muy probablemente el mapa final generado mejorará su capacidad de clasificación.

La arquitectura de análisis de los datos propuesta funcionó de manera adecuada ya que gracias al segundo nivel de clasificación por mapas auto-organizados, las categorías generadas sobre el mapa segmentado discriminaron de mejor manera los casos positivos de los negativos comparándolo con el desempeño del mapa observado durante el primer nivel de clasificación, teniendo que recurrir en menores ocasiones a la probabilidad asociada para cierta región ya que durante la validación, en la mayoría de los casos las etiquetas de los nodos activados establecían la condición del sujeto.

Por otra parte, respecto a la eficiencia en la clasificación del mapa final, el método contemporáneo ocupado para la extracción de características (rasgos wavelet) resultó ser un poco mejor que el método clásico (rasgos frecuenciales), esto, gracias a la comparación de las medidas estadísticas para la tasa de acierto arrojadas por el proceso de validación cruzada para ambos casos sin embargo, ambos prototipos mostraron relativamente buenos resultados permitiendo con ello pensar el posible uso, en un futuro, de estos tipos de sistemas cuantitativos durante la tarea diagnóstica para este trastorno neuropsicológico, ya que en ambos mapas la eficiencia en la clasificación fue muy aceptable.

Es posible que aquellos casos en los que el $S O M_{f}$ arrojó valores medios de probabilidad, se puedan atribuir a que los sujetos en tales circunstancias no tienen tan desarrollada la enfermedad o bien, a que han desarrollado mecanismos de adaptación que ocasionaron que los rasgos cerebrales analizados por el SOM sean similares a los de aquellos sujetos sin el padecimiento o incluso, a los continuos cambios en los parámetros de aceptación de la condición dados por el DSM.

\subsection{Conclusiones finales del trabajo}

Básicamente, después del desarrollo de todo este trabajo se pudieron establecer conclusiones sobre el posible uso del soporte computacional para estos tipos de desórdenes mentales

- Primeramente, los métodos para la extracción de rasgos propuestos, modelan adecuadamente la actividad eléctrica cerebral, observando que el algoritmo del mapa auto-organizado fue capaz de encontrar, a partir de ambos conjuntos de rasgos (rasgos frecuenciales mediante un enfoque clásico de análisis de potencias espectrales y rasgos wavelet con ayuda de un análisis multiresolución que permitió estudiar a las señales de EEG a un detalle igual que el 
ancho de banda del ritmo cerebral más lento), correlaciones en los datos de las poblaciones estudiadas

- Además, la tasa de acierto del clasificador fue relativamente alta (para datos no empleados durante el entrenamiento y validados a seis vías). Numéricamente se encontró para los rasgos frecuenciales valores de $\bar{t}_{a} \pm s_{t_{a}}=0,7 \pm 0,11$ y para los rasgos wavelet valores de $\bar{t}_{a} \pm s_{t_{a}}=0,77 \pm 0,15$ presentándose un mejor resultado en la clasificación ocupando la extracción de los rasgos mediante el análisis multiresolución.

- La metodología y arquitectura de análisis propuesta en esta tesis puede ser considerada para contribuir al diagnóstico a nivel poblacional del trastorno por déficit de atención con hiperactividad, ofreciendo un soporte con un enfoque puramente cuantitativo al experto clínico durante su tarea diagnóstica.

Pudieron lograrse cubrir de manera satisfactoria todos lo objetivos planteados al comienzo de esta investigación ya que la metodología seguida durante ésta, arrojó mapas finales capaces de clasificar adecuadamente a sujetos que presentan características propias del trastorno por déficit de atención con hiperactividad cuestión, que como ya se había mencionado, resultaba de mucho interés para la futura mejora en el diagnóstico, tratamiento y rehabilitación de estas condiciones en los años próximos.

Desafortunadamente, debido a que el número de individuos que constituyó a la población estudiada (28 sujetos en total) no fue tan grande como se hubiera deseado, posiblemente para poder mejorar los resultados de esta investigación solamente bastaría incorporar a ésta, una mayor cantidad de sujetos para ambas poblaciones (con y sin la patología) con el único fin, de ser capaces de generar con ello un mapa clasificador final $S O M_{f}$ que presente una mayor capacidad de generalización, es decir, que sea más robusto y confiable en su clasificación permitiendo de esta forma poder brindar un mejor y mas fiable soporte y finalmente tener las herramientas necesarias para establecer conclusiones más sólidas respecto a ésta propuesta de enfoque cuantitativo.

\subsection{Trabajo futuro y líneas de investigación abiertas}

Después de todos los resultados que se pudieron encontrar al realizar este proyecto, surgen gran cantidad de ideas relacionadas con el estudio, análisis y soporte al diagnóstico de desórdenes mentales que podrían elaborarse mediante un protocolo similar al presentado en esta tesis, el cual solamente se enfocó en determinar la posible existencia de sistemas computacionales capaces de ayudar en la clasificación de sujetos que presenten características relacionadas con el trastorno por déficit de atención con hiperactividad.

La idea que se propone a futuro para el soporte al diagnóstico consiste básicamente en que una vez generado el mapa clasificador final validado $S O M_{f}$ éste se utilice de manera paralela junto con el proceso cualitativo, ocupado actualmente para generar el diagnóstico de la patología 
en cuestión, tal y como se muestra esquemáticamente en la figura 9.1.

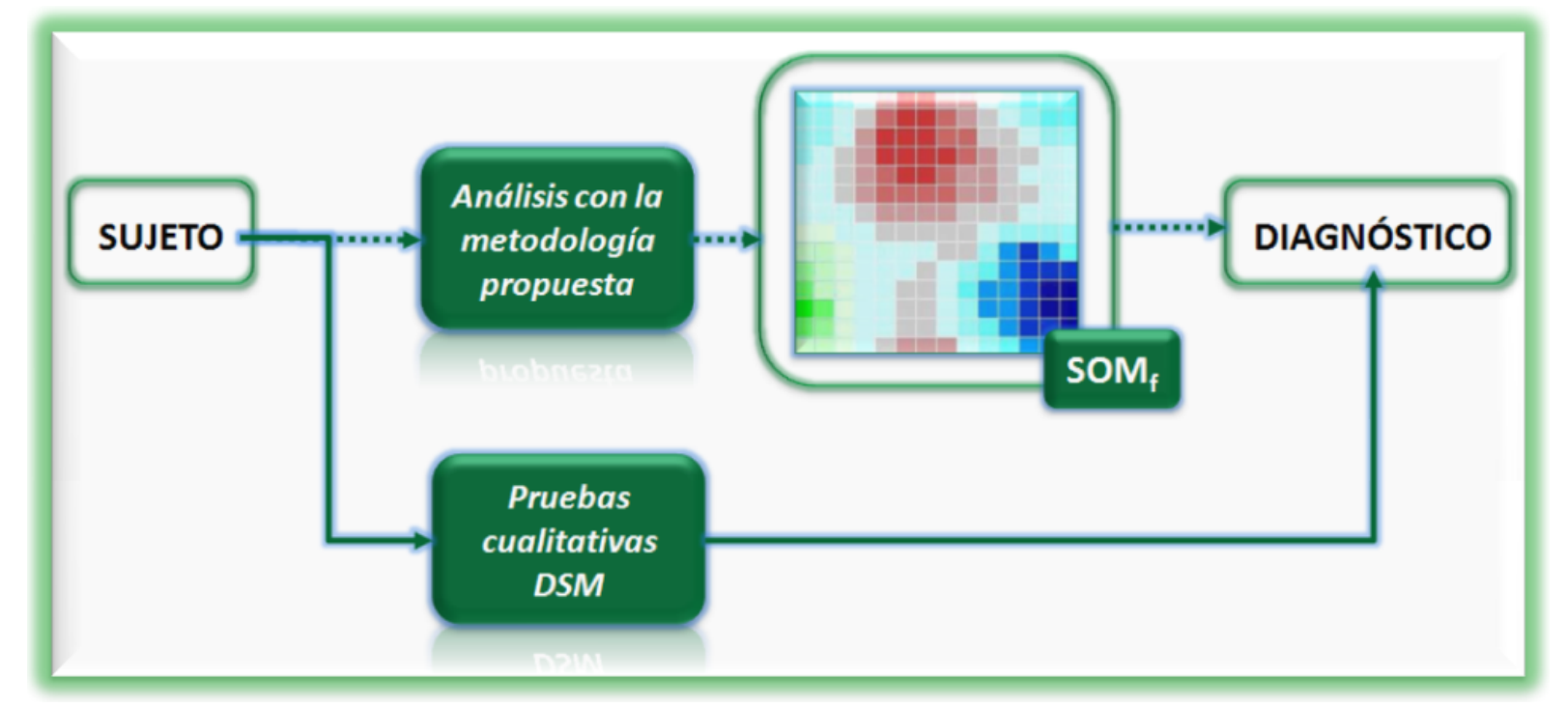

Figura 9.1:

Diagrama esquemático del proceso completo de diagnóstico del TDAH propuesto: trabajo conjunto de ambos proceso vía cuantitativa mediante el mapa clasificador (línea punteada) y vía cualitativa mediante las pruebas estandarizadas DSM (línea contínua).

Estos mapas también podrían ser ocupados para observar la evolución del medicamento proporcionado al paciente ya diagnósticado con TDAH, es decir, mediante estudios longitudinales aplicados al paciente determinar la efectividad del medicamento consumido (en cantidad y tipo) y evaluar si el sujeto es capaz de trasladarse de zonas de alta probabilidad a zonas con menor probabilidad.

Sin embargo, esta idea podría bien extrapolarse al estudio de otras patologías que tengan como médula o base la actvidad eléctrica cerebral medida por la electroencefalografía cuantitativa mencionando algunas como la epilepsia, la esquizofrenia, los trastornos de conducta y personalidad, la bipolaridad, entre muchas otras.

Se podría establecer un procedimiento para la generación de un gran mapa clasificador de desordenes mentales, construido con la información de sujetos que presenten todas estas patologías, el cual fuera capaz de discriminar no sólo a sujetos con o sin TDAH sino al contrario, también fuera capaz de colocar a un sujeto cualquiera en regiones relacionadas con alguna de las patologías mencionadas en líneas anteriores lo que sin duda alguna, resultaría interesante explorar. 


\title{
Apéndice A
}

\section{Electroencefalografía cuantitativa}

\author{
Por qué esta magnifica tecnología científica, \\ que ahorra trabajo y nos hace la vida mas fácil, \\ nos aporta tan poca felicidad? \\ La respuesta es simple: porque aún no hemos aprendido a usarla con tino.
}

ALBERT EINSTEIN

Hans Berger descubrió la actividad eléctrica del cerebro en 1923. Describió las señales electroencefalográficas como constituidas por cuatro diferentes ondas llamadas ritmos cerebrales, que presentan tanto diferentes frecuencias como amplitudes y que caracterizan a procesos diferentes tales como el sueño, el descansar, el despertar, algunas enfermedades mentales, entre otras.

\section{A.1. Principios basicos del EEG [24]}

Comunmente se describen ciertos tipos de ritmos cerebrales cuyas características más importantes son las mostradas en la tabla A.1.

Tabla A.1: Características más importantes de los ritmos cerebrales.

\begin{tabular}{|c|c|c|c|}
\hline Ritmo Cerebral & Frecuencia (Hz) & Amplitud $(\boldsymbol{m} \boldsymbol{V})$ & Región Predominante \\
\hline$\delta$ & $f<4$ & 100 & Frontal \\
$\theta$ & $4<f<8$ & $10-50$ & Temporal \\
$\alpha$ & $8<f<13$ & $20-50$ & Parietal/Occipital \\
$\beta$ & $f>13$ & $10-20$ & Frontal \\
\hline
\end{tabular}

La electroencefalografía cuantitativa a grandes rasgos consiste en la obtención de una representación gráfica de la diferencia de voltaje entre dos lugares diferentes del cerebro, trazado en el tiempo, a partir de uno o varios electrodos colocados sobre el cuero cabelludo o directamente en la corteza cerebral. La señal del EEG detectada en el cuero cabelludo es generada por las neuronas corticales representando así, la actividad eléctrica promedio de estas redes neuronales siendo modificado por propiedades eléctrico conductoras de los tejidos entre la fuente de potenciales y el electrodo en el cuero cabelludo, las propiedades conductoras del electrodo en sí, así como la orientación del generador cortical para el electrodo de registro. 
Un ejemplo de registro de EEG es el mostrado en la figura A.1, en la cual se puede apreciar que el registro intracraneal parece tener en general, actividad más regular y de mayor amplitud, mientras que la actividad registrada con electrodos en el cuero cabelludo parece ser atenuada [?].

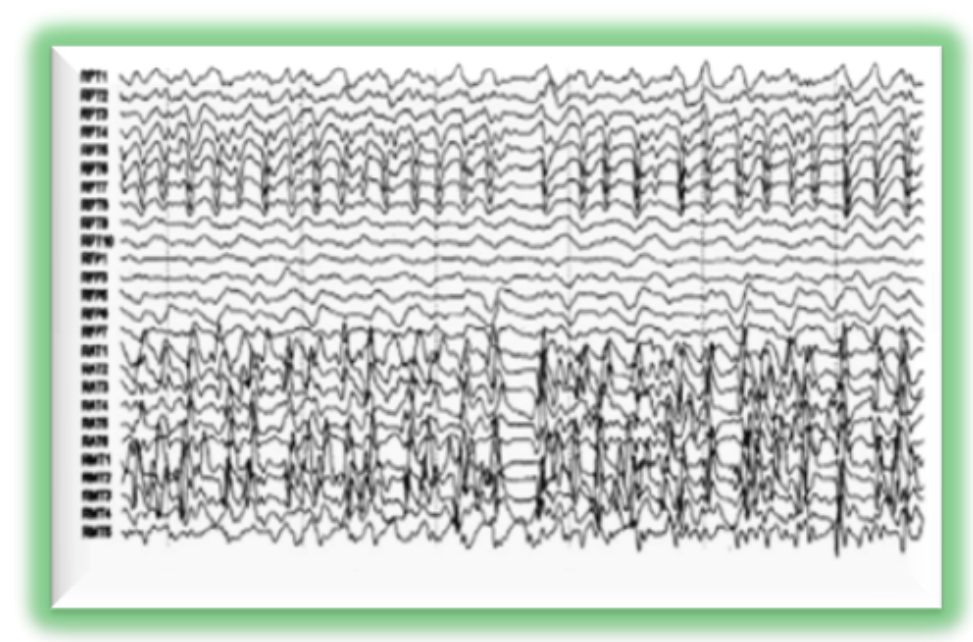

$a$

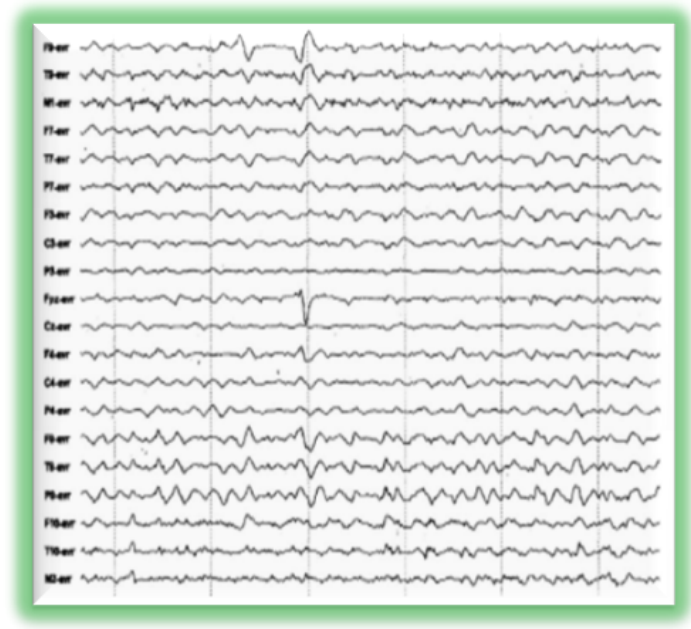

$b$

Figura A.1: Grabación simultánea del EEG en una cisura del lóbulo temporal.

a) Actividad eléctrica cerebral registrada intracranealmente.

b)Actividad eléctrica cerebral registrada en el cuero cabelludo.

La señal EEG puede ser obtenida por medio del registro del flujo de corriente a través de los tejidos entre el generador eléctrico y el electrodo de registro, lo cual se conoce como conducción de volumen. El EEG proporciona una proyección bidimensional de la realidad tridimensional, lo que significa que, teóricamente, es imposible determinar la ubicación del generador de la actividad registrada en el EEG basándose únicamente en la señal proveniente de los electrodos colocados en el cuero cabelludo, lo cual se conoce como el problema inverso en electroencefalografía.

\section{A.1.1. Fuentes sinápticas del EEG}

Para poder visualizar y monitorear el registro de la diminuta (en el rango de los microvoltios) actividad eléctrica cerebral, ésta debe ser duradera y vigorosa. Para decirlo de manera figurada, habría que encontrar una plataforma en la que tanto el examinador como el cerebro examinado se encontraran en el mismo tiempo-espacio continuo. Sólo cumpliría estos criterios la actividad sináptica y es la fuente más duradera de los potenciales del EEG. Cada sinapsis actúa como una batería generadora de corriente en un pequeño circuito cerrado. Tanto los potenciales postsinápticos excitatorios PEPS como los potenciales postsinápticos inhibitorios PIPS contribuyen a la actividad registrada como EEG.

La suma de las corrientes extracelulares es lo suficientemente lenta para generar potenciales de EEG. La corriente que fluye a través de la corteza cerebral, más la suma de la corriente eléctrica generada por las neuronas vecinas constituye una aproximación a la localización de la fuente de corriente eléctrica. Visto desde fuera de las células, las zonas de la membrana donde la corriente fluye dentro o fuera de las células se llaman, respectivamente, los sumideros y fuentes. 


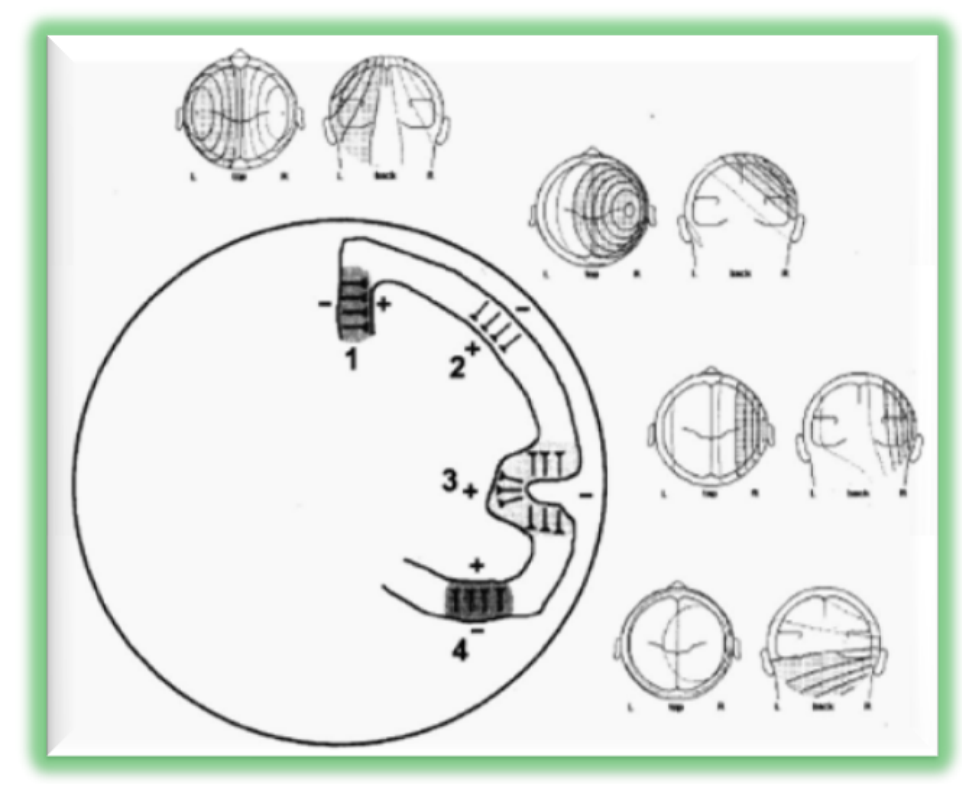

Figura A.2:

Esquema de una sección transversal del cerebro, que ilustra cuatro fuentes corticales representativas de la señal del EEG [?].

De la figura A.2, se puede apreciar que las fuentes dos y tres poseen campos de producción radial, por lo que el máximo voltaje negativo está directamente encima de ellos. Fuentes uno y cuatro producen campos tangenciales y tanto el voltaje máximo negativo, como positivo se desplazan a cualquier región.

Las corrientes de excitación, incluyen la participación de $\mathrm{Na}^{+}$e iones de $\mathrm{Ca}^{2+}$, el flujo hacia dentro, hacia el interior de la célula. La corriente de salida hace referencia a un retorno pasivo de corriente, del espacio intracelular al extracelular, y por otro lado las corrientes del circuito inhibitorio, incluyen la participación de los iones $\mathrm{Cl}^{+}$y K ${ }^{+}$, con flujo de corriente en la dirección opuesta.

Los electrodos del cuero cabelludo registran las diferencias de los potenciales que son causadas por los potenciales postsinápticos en la membrana celular de las neuronas corticales. Los circuitos cerrados de las líneas de trazos ligeros representan la suma de las corrientes extracelulares producida por los potenciales postsinápticos; los segmentos abiertos de las líneas de trazos más pesados conectan todos los puntos que tienen el mismo nivel de voltaje. Los dos electrodos en el cuero cabelludo tienen diferentes niveles de voltaje y registran esta diferencia. La actividad no-sináptica es una fuente menos importante del flujo de corriente extracelular que produce los potenciales de EEG. La actividad neuronal intrínseca, como los potenciales de acción rápida, usualmente tiene un periodo de duración demasiado corto como para tener un efecto en el EEG.

\section{A.1.2. Fuentes neuronales intrínsecas del EEG}

Los potenciales de acción rápida ( $\mathrm{Na}$ ), de corta duración (menores a dos $\mu \mathrm{s}$ ) y de gran amplitud, no contribuyen al registro de los potenciales en el cuero cabelludo, excepto durante eventos sincrónicos, tanto fisiológicos como los estados transitorios del sueño y patológicos, como la actividad epileptiforme. Los potenciales mediados por calcio, espiga de calcio, son dependientes del voltaje y son generados en forma sincrónica con los potenciales postsinápticos excitatorios 
dendríticos. Pueden contribuir a la creación de los sumideros de campo dendríticos, especialmente durante la actividad epileptiforme.

\section{Espigas Intrínsecas post-hiperpolarización}

La espiga intrínseca Post-hiperpolarización EIPH posterior a las espigas dendríticas de $\mathrm{Ca}^{2+}$, da por resultado la supresión de espigas rápidas e hiperpolarización de la membrana causada por la activación de la conductancia de $\mathrm{Ca}_{2}$, mediada por $\mathrm{K}^{+}$, las cuales son comparables en amplitud y duración con los eventos sinápticos y, como ellos, pueden contribuir con los potenciales de EEG registrados extracelularmente. Un ejemplo de lo anterior puede ser la generación de ondas $\delta$ en el sueño. En el cerebro despierto, neurotransmisores subcorticales como la acetilcolina, histamina y catecolamina reducen la conductancia de Ca mediada por K, bloqueando las EIPH relacionadas con las ondas $\delta$.

\section{A.1.3. Generadores corticales del EEG}

Las principales fuentes de EEG se derivan de la corteza cerebral y la forma tridimensional de los campos de potenciales, que son registrados como dos campos 3D proyectados en función del voltaje contra el tiempo. Se necesita de la actividad eléctrica sincrónica de alrededor de 108 neuronas en un área cortical de un mínimo de $6 \mathrm{~cm}^{2}$ para crear una señal visible en el EEG. El área de la corteza necesaria para la generación de espigas puede ser tan grande como de $20 \mathrm{~cm}^{2}$.

Como se mencionó anteriormente, los principales campos generadores de señales de EEG registrados en la superficie del cerebro o en el cuero cabelludo se clasifican en y PIPS de las neuronas piramidales. El sitio sináptico de los PEPS es un sumidero de corriente activa (campo negativo extracelular). Los iones positivos migran hacia la célula y despolarizan la membrana.

En la parte distal de la célula, el cuerpo y las dendritas distales, una fuente de corriente pasiva fuera de la célula está relacionada con el campo extracelular positivo. Los campos de EEG son generados principalmente por las neuronas piramidales grandes de orientación vertical situadas en las capas corticales III, V, y VI. Debido a las propiedades de atenuación del cráneo espacial tridimensional, la suma de la actividad cortical es fundamental para la producción de un campo de voltaje que pueda ser registrado en el cuero cabelludo.

\section{A.1.4. Potenciales de superficie y dendríticos postsinápticos}

El área generadora de actividad es fundamental para un electrodo cuando la orientación de la región cortical activa es de frente. Este es el caso cuando el campo de voltaje es radial y el electrodo está sobre la fuente generadora de actividad. Una fuente radial produce un campo de máxima actividad directamente sobre ella, y otro con la polaridad opuesta en el lado opuesto de la cabeza. Para una fuente superficial, el cuero cabelludo, más cercano es significativamente mayor que la que existe en el lado opuesto de la cabeza.

Un solo campo de voltaje máximo no puede ser utilizado para definir la orientación y/o localización de un generador de señal EEG cortical. A excepción de una fuente puramente radial, los principales campos generadores de una señal de EEG son desplazados desde una posición

directamente encima de él. La ubicación de los principales campos generadores tanto negativos 
como positivos y sus puntos fuertes deben ser considerados.

\section{Propagación de la actividad cortical}

Con la propagación de la actividad eléctrica en las regiones corticales adyacentes, la geometría de la actividad de origen cambia. Esta nueva ubicación y orientación de la actividad de origen ocupa un campo de voltaje diferente. El movimiento de la actividad en diferentes áreas del cuero cabelludo, que se presenta en decenas de milisegundos, sugiere la propagación de la fuente generadora de la actividad en el cerebro.

\section{Sincronización subcortical del EEG}

El tálamo dorsal es considerado como el principal generador de la actividad subcortical que registra el EEG, sincronizando grupos de neuronas neocorticales como generadores de voltaje. En condiciones normales, los núcleos del tálamo y las regiones corticales interactúan para producir la sincronía de los potenciales corticales postsinápticos, durante la vigilia y el sueño. De acuerdo con la teoría del marcapasos facultativo, las neuronas tálamo-corticales de relevo envían fibras a la corteza, y también envían ramificaciones de regreso y terminaciones en las neuronas inhibitorias talámicas.

La hipótesis del núcleo reticular talámico atribuye a éste la función de marcapasos, cuyas células liberan el neurotransmisor inhibidor GABA en secuencias rítmicas de despolarización dirigidas a las neuronas del tálamo dorsal y de la parte anterior del tronco encefálico. Además del ritmo $\alpha$ dominante generado en regiones occipitales, los ritmos de frecuencia $\alpha$ se generan en múltiples áreas corticales. Las conexiones corticales parecen ser más importantes en la generación de la mayoría de los ritmos $\alpha$, aunque las descargas tálamo-corticales pueden tener una influencia limitada en la posterior generadora del ritmo $\alpha$ dominante. Los ritmos rápidos en estado de vigilia, como las frecuencias $\beta$ y $\gamma$ son producidos por los generadores corticales. La entrada de colinérgica pontina al tálamo inhibe la producción de los generadores transitorios del sueño, como tales, los husos de sueño y las ondas $\delta$ de amplitud alta.

Los husos de sueño parecen ser fenómenos tálamo-corticales. Las neuronas GABAérgicas del núcleo reticular talámico descargan en ciclos rítmicos. Se proyectan a las neuronas tálamocorticales de relevo, que a su vez se proyectan principalmente hacia las neuronas corticales. La descarga de las neuronas de relevo después de la liberación del neurotransmisor GABA disminuye la inhibición. Lo que da como resultado la sincronización de los PEPS en la corteza, registrados por el EEG. A diferencia de los husos de sueño, que requieren actividad sináptica para establecer la oscilación rítmica, la frecuencia delta es un ritmo intrínseco que depende de los flujos de potasio en los canales de iones de las neuronas corticales y talámicas.

\section{Desincronización subcortical del EEG}

La desincronización del EEG es la interrupción de la actividad rítmica. Se produce por la activación de las proyecciones colinérgicas ascendentes del prosencéfalo basal, del tallo cerebral y por proyecciones de los núcleos del rafe y del locus coeruleus. La actividad rítmica es interrumpida por los efectos directos sobre las neuronas corticales e indirectamente sobre las neuronas del tálamo. A nivel celular, a la par con el proceso de desincronización, ocurre la transición de un 
patrón de disparo a un patrón de una o varias ráfagas de disparo. La desincronización aumenta con el estado de vigilia y es suprimida por el sueño no-REM. Algunos patrones sincrónicos anormales, como el coma $\alpha$, se producen en el contexto de daños generalizados a los grupos neuronales ascendentes que de otra manera producen el estado de vigilia y desincronización.

\section{A.2. Sistema Internacional de Colocación de Electrodos 10/20 para registro de EEG}

Durante la decada de 1950, el Dr. Herbert Jasper $5^{5}$ desarrolló un sistema lógico denominado sistema de colocación de electrodos 10/20 el cual depende de la realización de mediciones precisas en el cráneo utilizando diversas marcas anatómicas; básicamente se obtienen mediciones en tres planos (sagital, coronal y horizontal) de un arco semicircuferencial del cráneo siendo éste aceptado de manera genérica.

- La medición sagital se extiende desde el nasión ${ }^{6}$ en la zona superior de la cabeza hasta el inión7. Esta medida define el plano sagital mostrado en la figura A.3

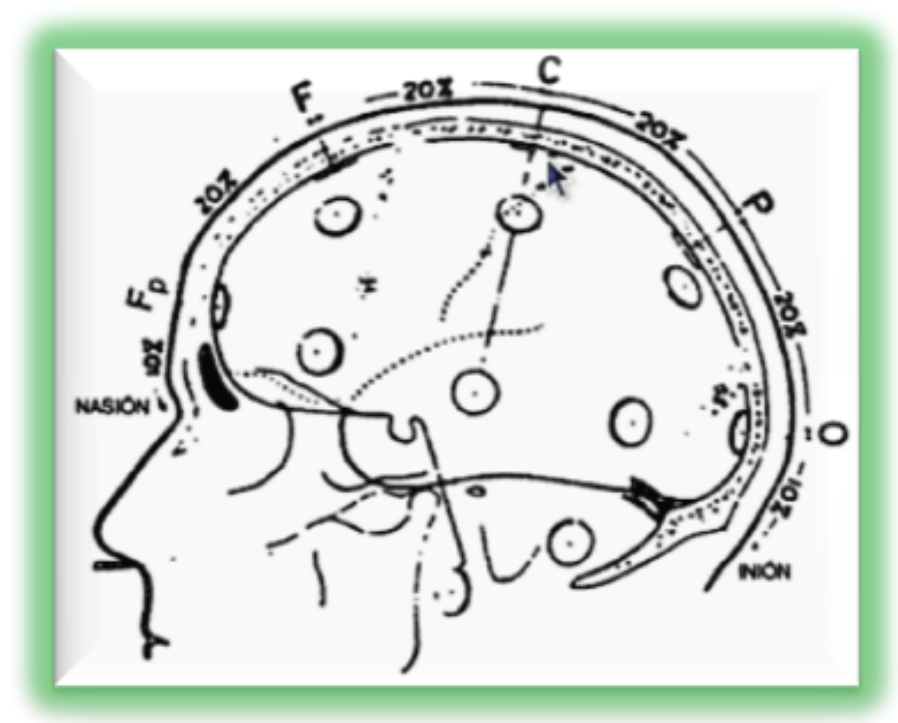

Figura A.3:

Imagen lateral del cráneo que muestra las mediciones desde el nasión hasta el inión en la línea media. $F_{p}$ es la posición en el polo frontal, $F, C, P$ y $O$ son las líneas frontales, centrales, parietales y occipitales de electrodos respectivamente. Los porcentajes indicados representan las proporciones de la distancia medida desde el nasión hasta el inión. Se puede apreciar quela línea central corresponde a un $50 \%$ de esta distancia y que los electrodos de los polos frontal y occipital representan un $10 \%$ de la distancia entre el nasión y el inión respectivamente. La línea externa de electrodos está separada el doble de esta distancia, es decir, un $20 \%$.

(Reproducida de Report of the committee on methods of clinical examination in electroencephalography. Electroenceph Clin Neurophysiol 1958; 10:370-375)

Ahora, hay tres mediciones que permiten definir a los otros dos planos, la primera es la mitad de la distancia entre el nasión y el inión, que representa uno de los marcadores del vértex, las otras dos mediciones son dos puntos que quedan por encima del nasión y el inión, que constituyen cada uno el $10 \%$ de la medición sagital total y que en total facilitan el establecimiento del plano horizontal.

\footnotetext{
${ }^{5}$ Renombrado especialista en electroencefalografía del Montreal Neurological Institute

${ }^{6}$ Depresión situada en la parte superior de la nariz

${ }^{7}$ Prominencia existente en la línea media de la base del occipucio
} 
- La medición coronal se extiende desde un punto inmediatamente anterior al tragd $8^{8} \mathrm{y}$ atraviesa el punto medio de la medición sagital hasta el mismo punto del lado opuesto. Esta medición define el plano coronal. La intersección de los puntos medios de las mediciones sagital y coronal representan la localización del vértex y, por lo tanto, del electrodo $C_{z}$ mostrado en la figura A.4-a

Los primeros puntos correspondientes al $10 \%$ definen los electrodos $T_{3}$ y $T_{4}$, que son los electrodos temporales mediales; los puntos siguientes correspondientes al $20 \%$ definenlos electrodos centrales $C_{3}$ y $C_{4}$. Los segmentos restantes correspondientes al $20 \%$ representan la distancia entre $C_{3}$ y $C_{4}$ por un lado y $C_{z}$ por otro.

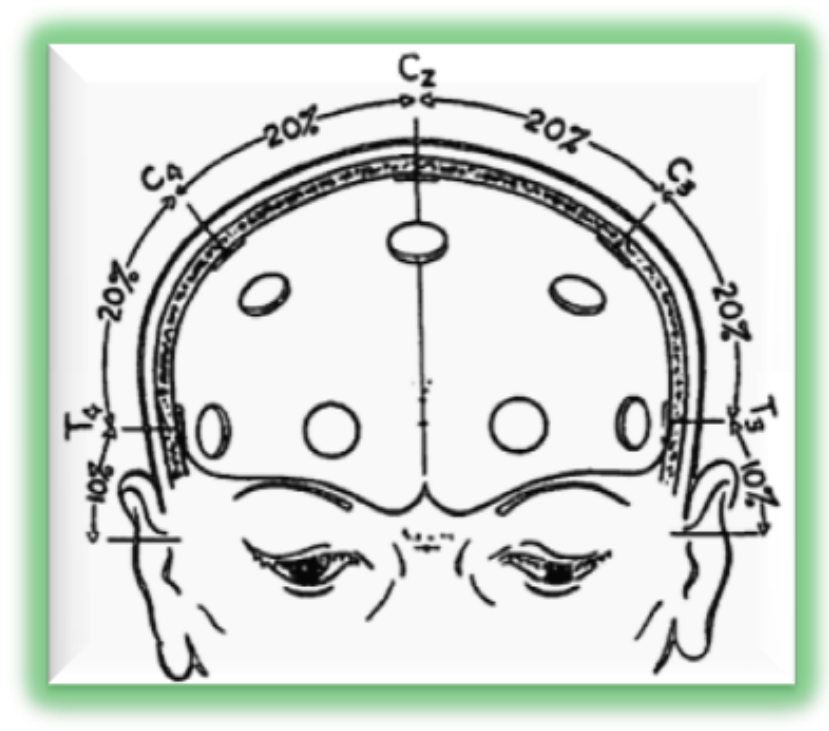

$a$

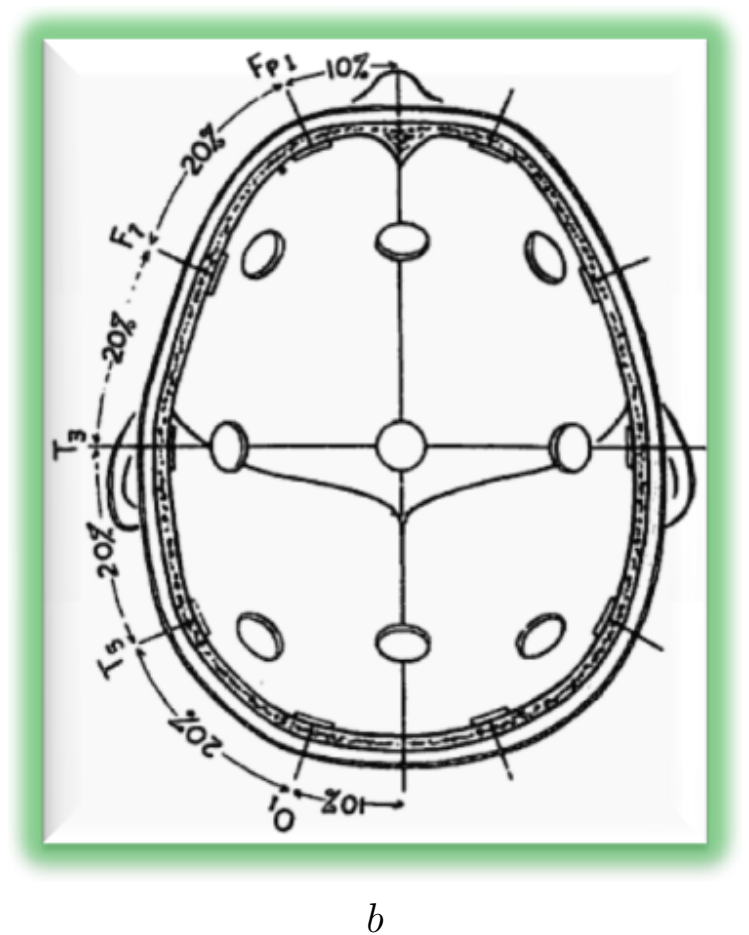

$b$

Figura A.4: Mediciones para la determinacion de los planos coronal y horizontal (Reproducida de Report of the committee on methods of clinical examination in electroencephalography. Electroenceph Clin Neurophysiol 1958; 10:370-375) a)Imagen frontal del cráneo que muestra el método de medición de la línea central de electrodos.

b) Imagen superior con sección transversal del cráneo a través de la línea temporal de electrodos que ilustra el sistema 10/20.

Para definir la medición horizontal se utiliza el punto que queda por encima del trago a cada lado (y que represental el $10 \%$ de la medición coronal total) y los puntos situados inmediatamente encima del nasión y el inión, que representan el $10 \%$ de la medición sagital total

- La medición horizontal de obtiene por separado en ambos lados de la cabeza, se extiende desde un punto por encima del nasión atravesando el punto que queda por encima del trago hasta el punto situado por encima del inión. Estas mediciones definen al plano horizontal mostrado en la figura A.4-b

Los puntos del plano horizontal correspondientes al $10 \%$ definen los electrodos $F_{p 1}$ y $F_{p 2}$ en la parte anterior (frontopolares) y los electrodos $O_{1}$ y $O_{2}$ en la parte posterior (occipitales). Los

\footnotetext{
${ }^{8}$ Protrusión cartilaginosa situada en la parte anterior del oido externo
} 
puntos del $20 \%$ definen los electrodos $F_{7}$ y $F_{8}$ en la parte anterior (frontales inferiores) y los electrodos $T_{5}$ y $T_{6}$ en la parte posterior (temporales posteriores). Los dos segmentos restantes correspondientes al $20 \%$ representan las distancias $F_{7}-T_{3}$ y $T_{3}-T_{5}$ en la parte izquierda y $F_{8}-T_{4}$ y $T_{4}-T_{6}$ en la derecha.

Los electrodos $F_{3}$ y $F_{4}$ se definen por los puntos situados a la mitad de la distancia entre $F_{7}$ y $F_{z}$ a la izquierda y entre $F_{8}$ y $F_{z}$ a la derecha. De la misma manera, $P_{3}$ y $P_{4}$ quedan definidos por los puntos medios entre $T_{5}$ y $P_{z}$ a la izquierda y entre $T_{6}$ y $P_{z}$ a la derecha.

Finalmente, las localizaciones y definicones estándar para cada uno de los electrodos, que conforman al sistema 10/20, se exponen a continuación: (Figuras A.5-A.7)

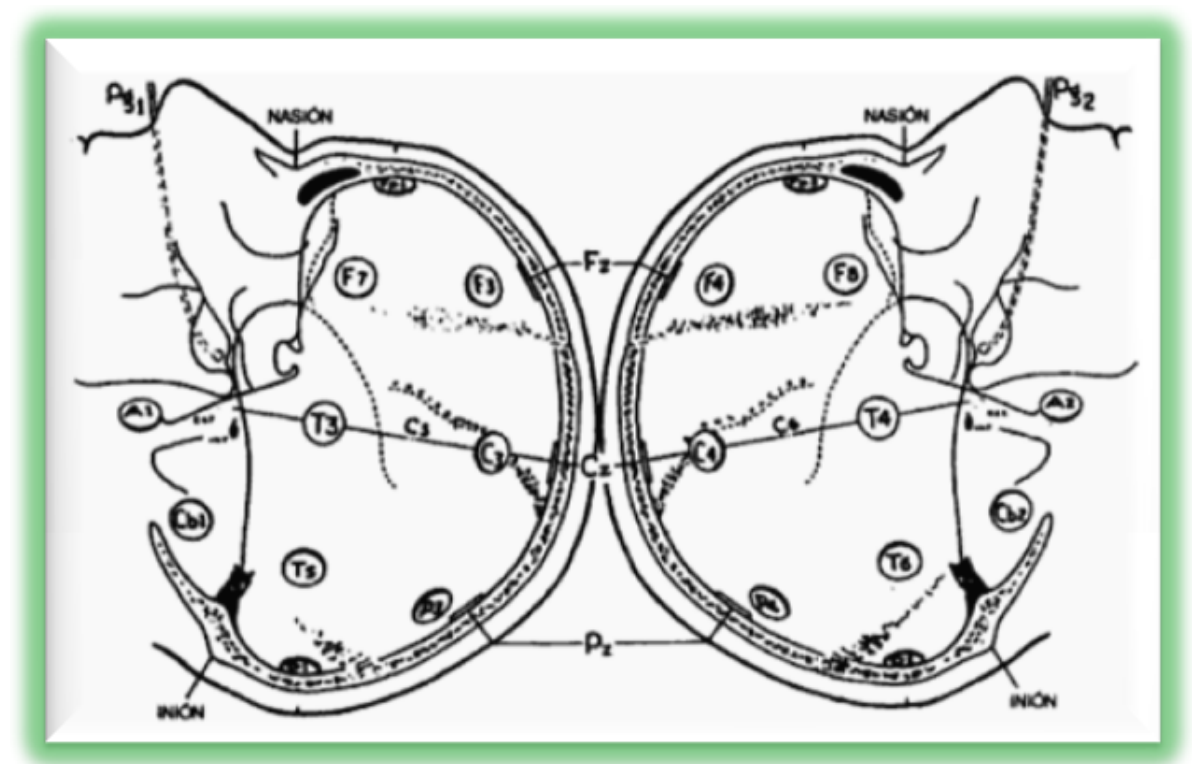

Figura A.5:

Imagen lateral de los hemisferios izquierdo y derecho en la que aparecen todas las posiciones estándar de los electrodos con omisión de las posiciones intermedias, $C_{5}$ y $C_{6}$. Estos esquemas se obtuvieron a partir de una serie de radiografías con proyecciones laterales verdaderas. (Reproducida de Report of the committee on methods of clinical examination in electroencephalography. Electroenceph Clin Neurophysiol 1958; 10:370-375)

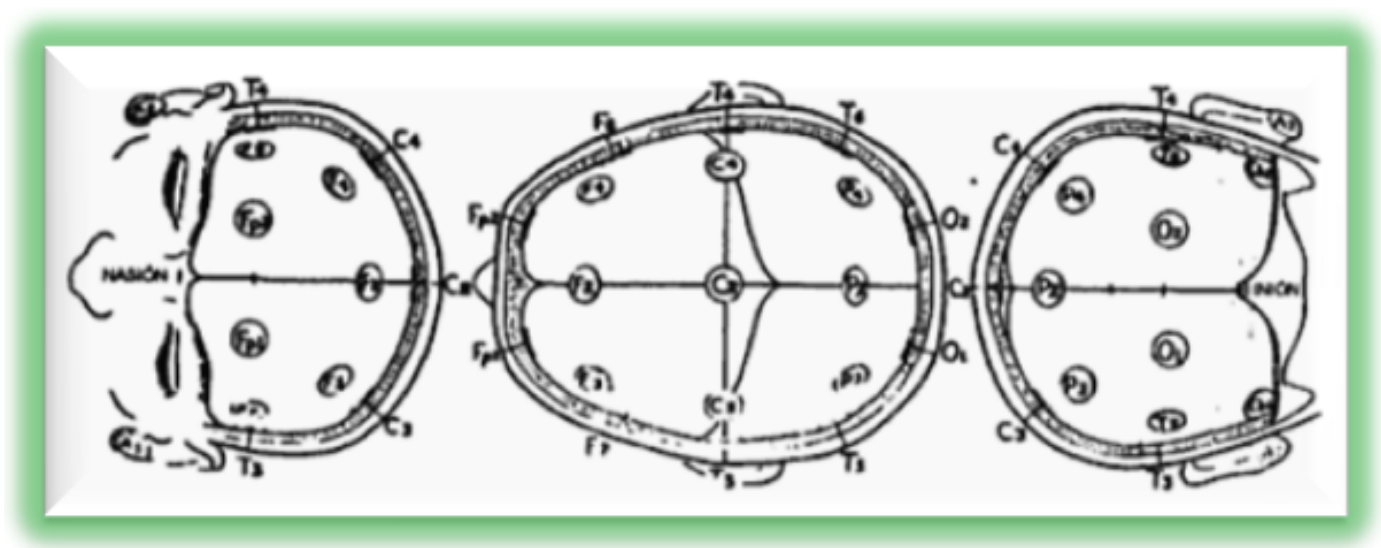

Figura A.6:

Imagen frontal superior y posterior en las que se observan todas las posiciones de los electrodos estándar. (Reproducida de Report of the committee on methods of clinical examination in electroencephalography. Electroenceph Clin Neurophysiol 1958; 10:370-375) 


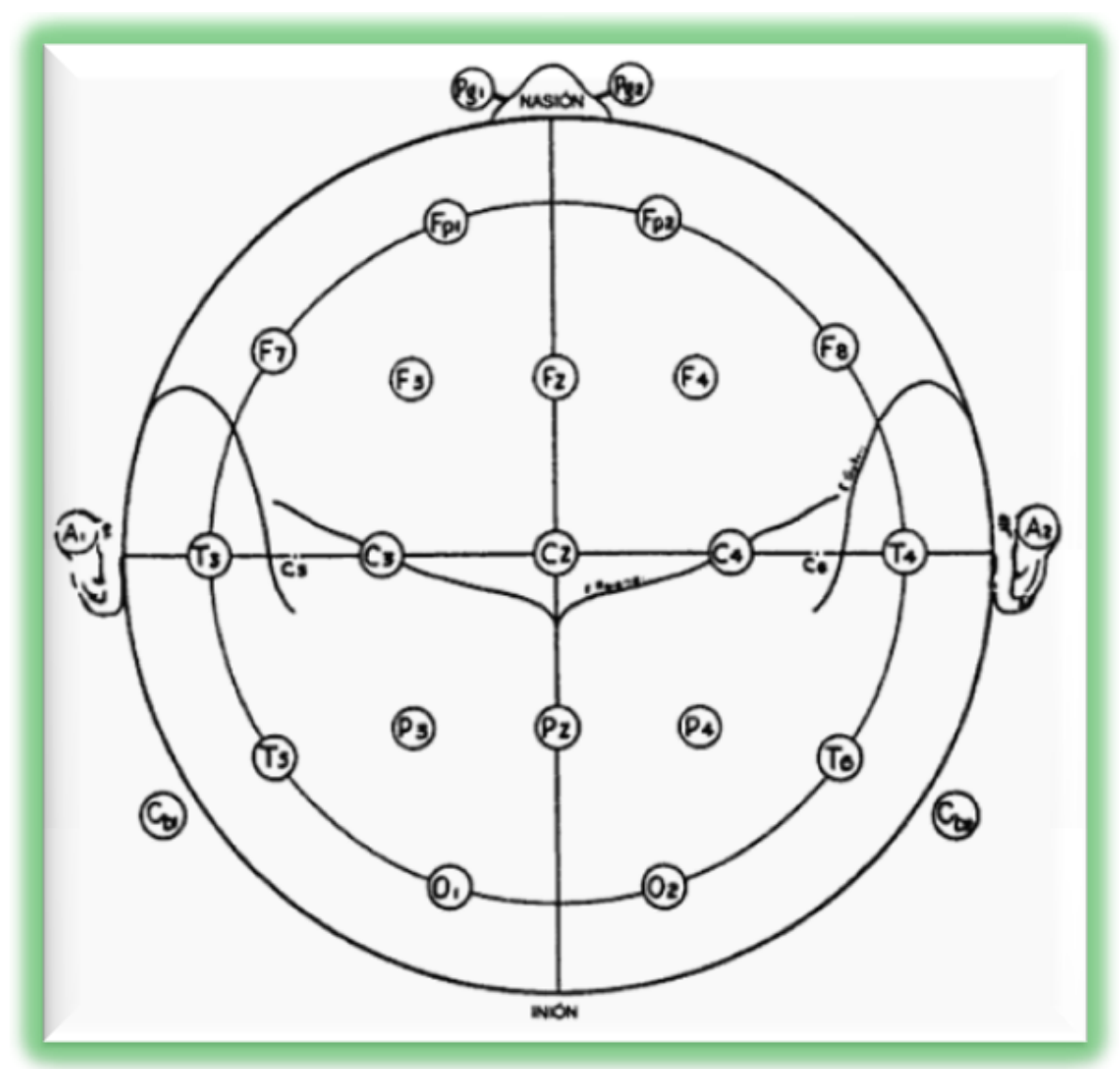

Figura A.7:

Proyección en plano único de la cabeza con demostración de todas las posiciones estándar y con las localizaciones correspondientes a las cisuras de Rolando y Silvio. El círculo externo fue trazado a nivel del nasión y el inión, el círculo interno representa la línea temporal de elctrodos. Este esuqema es una representación útil para la indicación de la colocación de los electrodos en los registros sistemáticos. (Reproducida de Report of the committee on methods of clinical examination in electroencephalography. Electroenceph Clin Neurophysiol 1958; 10:370-375)

- $F_{z}, C_{z}, P_{z}$ : Electrodos de la línea media en las regiones frontal, central y parietal, registran los potenciales procedentes de las superficies de la línea media y mediales de los hemisferios.

- $\frac{A_{1}}{A_{2}}$ : Electrodos de referencia en los pabellones auriculares y aunque son utilizados como referencia, también registran la actividad procedente de las regiones temporales mediales.

- $\frac{F_{p 1}}{F_{p 2}}$ : Prefrontal, ubicada en la frente, registra la actividad de los polos frontales

- $\frac{F_{3}}{F_{4}}$ : Mediofrontal, ubicada sobre el lóbulo frontal, registra la actividad frontal

- $\frac{C_{3}}{C_{4}}$ : Central, ubicada sobre la cisura de Rolando denominada surco central

- $\frac{P_{3}}{P_{4}}$ : Parietal, registra la actividad parietal

- $\frac{O_{1}}{O_{2}}$ : Occipital, registra la actividad occipital

- $\frac{F_{7}}{F_{8}}$ : Frontal inferior, registra la actividad de las regiones orbitofrontal, frontal lateral y temporal anterior

- $\frac{T_{3}}{T_{4}}$ : Temporal medial, registra la actividad de las regiones temporales anterior y medial

- $\frac{T_{5}}{T_{6}}$ : Temporal posterior, registra la actividad en las regiones temporales posteriores 


\section{Apéndice B}

$32^{\text {nd }}$ Anual International Conference of the IEEE Engineering in Medicine and Biology Society, EMBC 2010.

Si buscas resultados distintos, no hagas siempre lo mismo. ALBERT EINSTEIN

Assisted Diagnosis of Attention-Deficit Hyperactivity Disorder through EEG Bandpower Clustering with Self-Organizing Maps. 


\title{
Assisted Diagnosis of Attention-Deficit Hyperactivity Disorder through EEG Bandpower Clustering with Self-Organizing Maps
}

\author{
Federico Alba-Sanchez ${ }^{a}$ Oscar Yanez-Suarez ${ }^{a}$ Hector Brust-Carmona $^{b}$ \\ ${ }^{a}$ Neuroimaging Laboratory, Universidad Autonoma Metropolitana - Iztapalapa, Mexico. \\ ${ }^{b}$ National Rehabilitation Institute, Mexico.
}

\begin{abstract}
The electroencephalogram is an attractive clinical tool given its non-invasive nature, its ability to reflect realtime changes in local cortical activity, and the load of objective bioelectrical measurements that can be derived from it. For decades, the electroencephalogram has been successfully used for diagnosing epilepsy and schizophrenia, among other brain disorders. This paper focuses in the design and implementation of a computer-aided diagnostic tool for establishing the likelihood of presence of Attention-Deficit Hyperactivity Disorder in children, out of routine electroencephalographic recordings obtained during a specific visual stimulation protocol. Classical bandpower features from multiple differential recordings are computed and used as features in a classifier built from a cooperative ensemble of labeled self-organizing maps. Classification accuracy of the proposed system is $0,7 \pm 0,11$, as estimated from unseen data, a result that points to the idea that such a quantitative diagnostic aid could adequately support the diagnostic task of a clinical expert.
\end{abstract}

\section{INTRODUCTION}

The Attention-Deficit Hyperactivity Disorder (ADHD) is one of the most common psychiatric conditions among children in the 6-17 age range, who present varying degrees of impulsiveness, lack of attention and hyperactivity (conditions that change as the children become of age). This disorder interferes with normal child development and, if missed for timely treatment, will preset the patient for problematic personal, social and psychiatric issues, while significantly deteriorating some behavioral and cognitive functionalities [1].

Patients who have been clinically diagnosed with ADHD through standard psychological testing and family interviews, show deficits similar to those of patients with frontal lobe lesions when performing tasks that require continued attention [2]. Recent electroencephalograpic (EEG) research has found consistent differences in activity among children with and without ADHD. Those children who have been diagnosed with the disorder typically show excess power in low frequency $\operatorname{rhythms}(\delta, \theta)$ with a corresponding reduction in power for higher frequency bands $(\alpha, \beta)$, when compared to normal subjects under closed-eyes condition [2]. Moreover, a significantly larger percentage of epileptoid activity has been observed in diagnosed children, being more evident during maneuvers such as hyperventilation or photostimulation, with additional

Corresponding author: F. Alba-Sanchez, chambridubri@gmail.com presence of inter-hemispheric anormalities such as power asymmetry or reduced parieto-temporal and increased fronto-central coherences [3]. Additional reports strenghten the assumption that subjects with ADHD might have an overall reduction of their cortical activation level, supporting the hypothesis of a maduration delay within the frontal neural systems [4], that is, that when facing a demanding cognitive task there is no evidence of the expected increase of high frequency rhythms.

Current ADHD diagnosis is based mainly in observations of children behavior as reported by parents and/or teachers through standardized questionnaires such as DSM-IV and WISC [5], which are highly subjective. The need for an objective and independent evaluation is relevant so that a confident decision about the presence or absence of this disorder could be established. Given that ADHD is indeed the result of central nervous system disfunction [6], and considering that EEG provides a continuous measure of cerebral activity, it is reasonable to think that this bioelectrical potential carries information that could be used within an adequate decision-support tool for objective evaluation. If better diagnosis is realized, better medication schemes or even neural rehabilitation procedures might be devised in the future.

This paper discusses the design and performance of a computational intelligence tool for objective support of the ADHD clinical expert decision. This tool uses bandpower features derived from multi-channel differential EEG recordings as inputs to a self-organizing map (SOM [7]) ensemble, providing a multivariate classification analysis framework. SOM output is presented in a simple visual indication of confidence about healthy or affected conditions.

\section{METHODOLOGY}

\section{Subjects}

Studied population consisted of 28 children with ages between 7-12 years, eighteen of which were diagnosed with ADHD (15 male, three female) and ten without ADHD (five male, five female). Subjects were referred by their educational institutions for psychological evaluation and ADHD diagnosis to the Neuropsychology section of the National Rehabilitation Institute in Mexico City. Evaluation 
was performed through the application of standardized DSMIV questionaries [5] and a clinical interview. EEG recordings were carried out at the Electrodiagnosis service of the same Institute, by means of a NicoletOne cEEG system (VIASYS Healthcare, USA).

\section{Stimulation Protocol}

Visual stimulation was carried out through the application of 20 bursts of 10 flashes each, with an inter-stimulus interval of 0.2 seconds, as shown in Fig. 1.

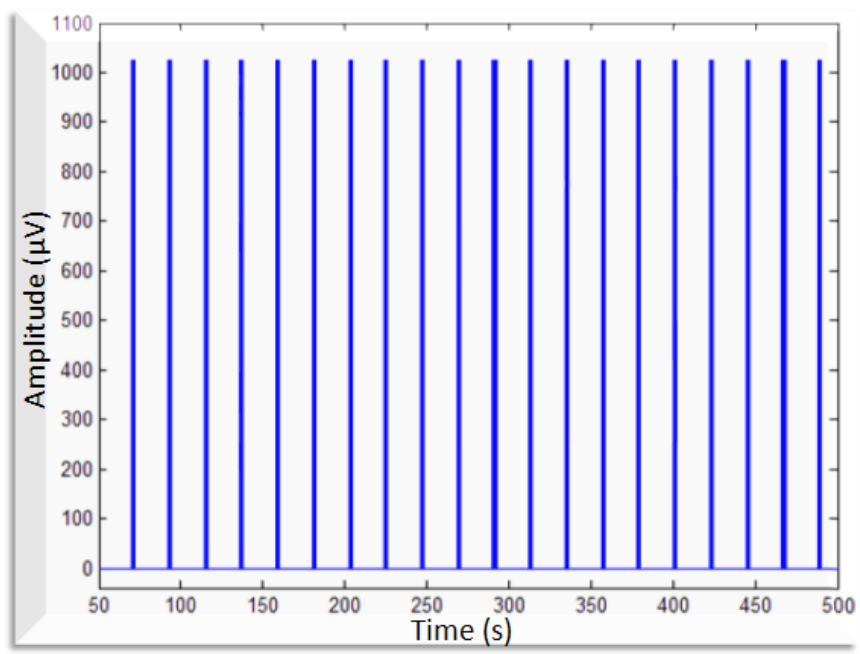

(a) Train of twenty photostimulations.

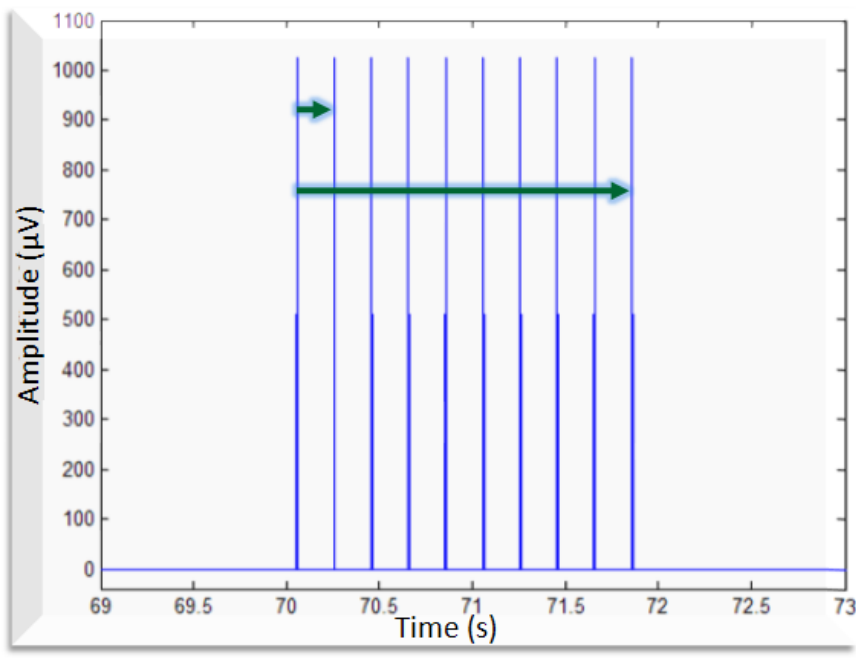

(b) First ten flashes corresponding to the first photostimulation pulse.

Fig. 1. Visual stimulus specification

\section{Signal Recording}

Subjects were placed in supine position, and asked to remain awake but relaxed with closed eyes. Ten monopolar scalp electrodes corresponding to the standard 10/20 locations $F_{p 2}, F_{4}, C_{4}, P_{4}, O_{2}$ (right hemisphere RH), $F_{p 1}$, $F_{3}, C_{3}, P_{3}$, and $O_{1}$ (left hemisphere $\mathrm{LH}$ ) were used for recording and digitization at a sampling frequency of 256 $\mathrm{Hz}$, with a bandpass filter in the $[0.5,40] \mathrm{Hz}$ range.
Offline digital substraction of adjacent intrahemispheric signal recordings was carried out to end up with eight synthetic bipolar recordings to be processed (Fig. 2)

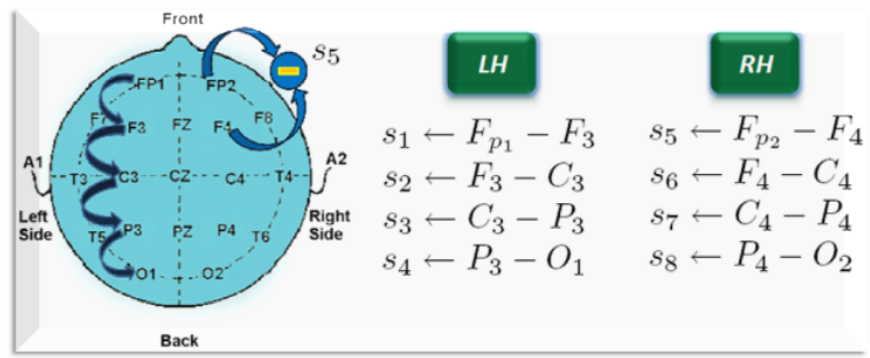

Fig. 2. Scheme of the acquisition process of the EEG recordings.

\section{Processing}

Digital signal processing of the eight bipolar recordings included the following steps:

- Filtering through a filter bank of four 100th-order symmetric FIR filters [8] to retrieve bandlimited signal versions: $\delta$ [0.5-4] Hz, $\theta$ [4-8] Hz, $\alpha$ [8-13] Hz, and $\beta$ [13-40] Hz, thus obtaining 32 signals in total.

- Segmentation, or selection of three signal intervals per burst stimulation: pre-stimulus, trans-stimulus, and post-stimulus, two seconds each, for a total of 1920 signal segments per subject as shown in Fig. 3.

- Feature extraction, computing average power of each segment through trapezoidal integration of the Welch periodogram [8].

- Feature vector formation, collecting the power indexes $P$, per cerebral wave, into vectors $x_{\text {band }}$ as in (1).

$$
x_{\text {band }}=\left\{P_{i, j, k}\right\} \in \mathbb{R}^{480} \quad \forall \quad \begin{gathered}
i=\text { pre, trans, post } \\
j=1,2, \cdots, 20 \\
k=1,2, \cdots, 8
\end{gathered}
$$

where $i$ denotes temporal location of the corresponding segment, $j$ corresponds to the burst repetition, $k$ indicates the bipolar recording and band represents each cerebral rhythm $(\delta, \theta, \alpha$ and $\beta)$.

\section{ENSEMBLE OF SELF-ORGANIZING MAPS}

The SOM is an artificial neural network model that establishes a projection from a high-dimensional input space (like the feature space just described) into a topologically ordered two-dimensional grid of nodes or neurons $n_{i j}$. Each node has an associated weight vector $\omega_{i j}=\left\{w_{i j_{1}}, w_{i j_{2}}, \cdots, w_{i j_{N}}\right\}$, which connects the input vector to the node; every node is therefore connected to the map input. The ensemble of all these vectors $\omega_{i j}$ form the codebook of the map. Among all the nodes of the two-dimensional array, implicit excitation and inhibition 


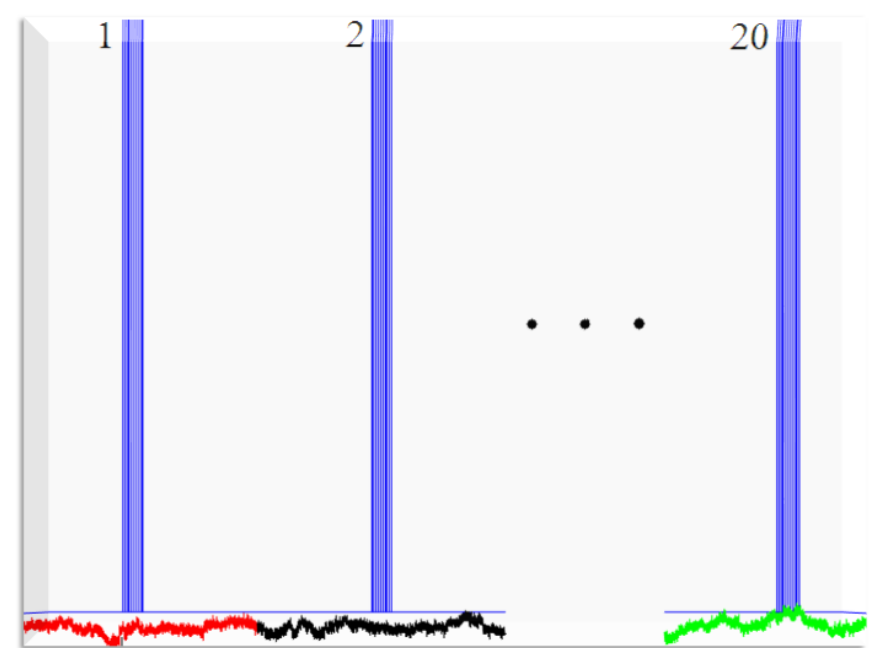

(a) Segmentation per each of the 20 repetitions of stimulation.

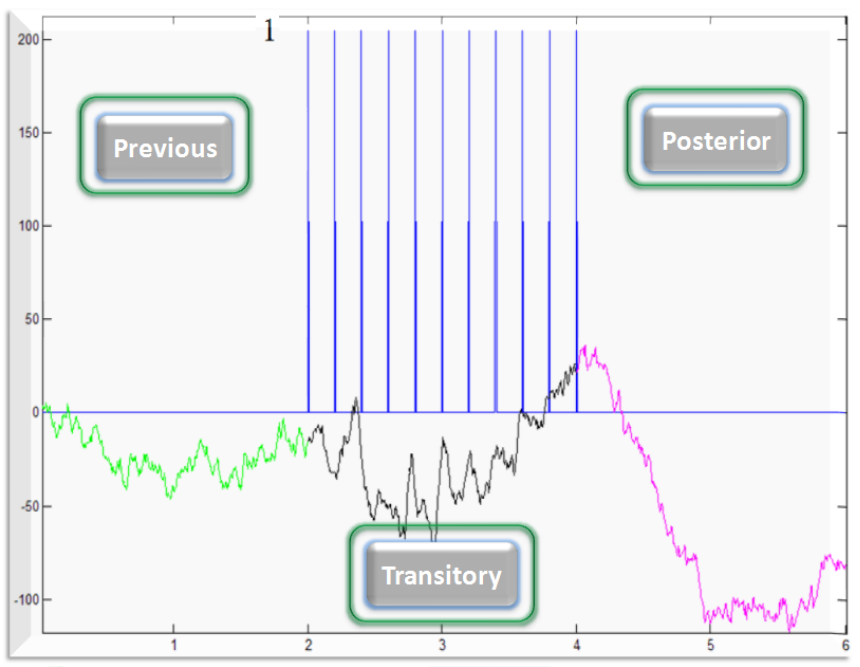

(b) Pre-, trans-, and post-stimulus segments

Fig. 3. Signal segmentation in relation to stimulation.

connections are established, because although they are not physically connected, each one has some influence over its neighbors. Neighbor influence is determined by the application of a function called neighborhood kernel, $h_{a b}(t)=h\left(r_{a}, r_{b} ; t\right)$, where $r_{a}$ and $r_{b}$ are the locations in the grid of nodes $a$ and $b$, and $t$ is the training time coordinate $[7,9]$. If the map inputs $x_{\text {band }} \in \mathbb{R}^{480}$ are stochastic vectors, assumed to be drawn from the probability density function $p\left(x_{\text {band }}\right)$, then, during the training of the SOM, the codebook vectors are moved so that most of them settle in areas where the density of the data is high while fewer are found in areas where low data occurrences exist.

\section{Classifier Architecture}

The SOM ensemble designed for classification consists of two levels of 10x10 node SOMs. The first level maps $\left(S O M_{\delta}, S O M_{\theta}, S O M_{\alpha}\right.$ and $\left.S O M_{\beta}\right)$ are trained on pre, trans-, and post- bandpower features for each band. For a given input vector $x_{\text {band }}$, its corresponding map will produce a single active node, whose coordinates, $y_{\text {band }}$, are arranged into vector $y=\left\{y_{\delta}, y_{\theta}, y_{\alpha}, y_{\beta}\right\}$. These coordinate vectors are used as features for the second-level map, $S O M_{f}$ (Fig. 4).

The codebook from the trained $S O M_{f}$ is labeled for classification in a two step procedure. First, the codebook is clustered using the $k$-means algorithm [10], choosing $k$ as the one that yielded the minimum sum of squared errors. This clustering determines groups of nodes with similar codebook vectors. Figure 5 displays $S O M_{f}$ nodes in a grid, color-coded by cluster. Second, the probability of a cluster representing a positive ADHD case is computed by counting the activations within the cluster when the map is presented with the labeled training set. Two thresholds of probability are arbitrarily chosen to define each cluster as corresponding to high-, medium- or low-confidence of representing positive ADHD cases. The bottom portion of Fig. 5 shows the cluster probabilities sorted from left to right, together with dashed lines dividing the confidence labels.

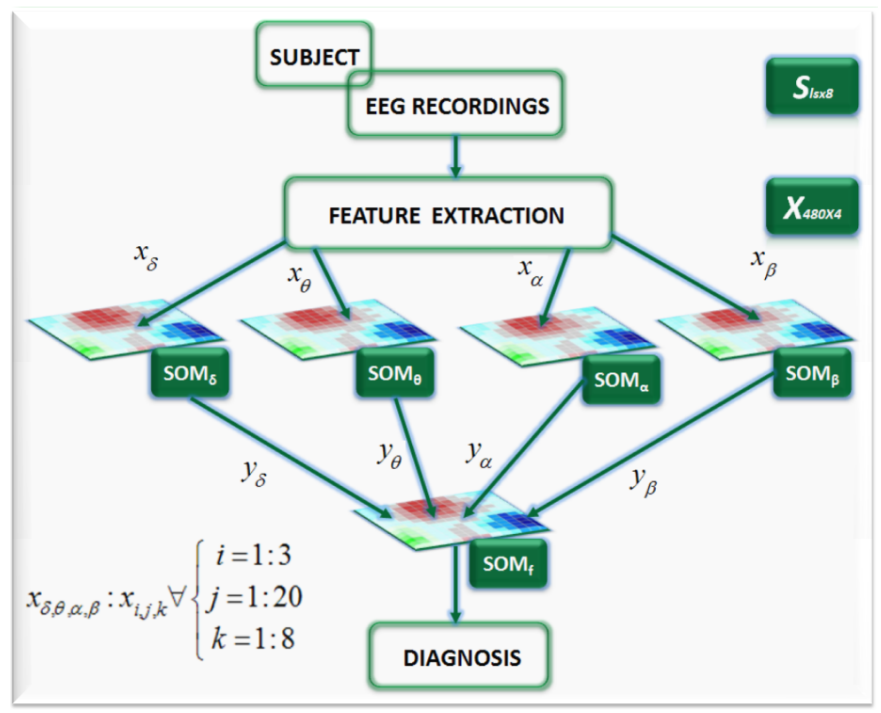

Fig. 4. Diagram for the proposed analysis of the extracted features.

In designing the classifier, a six-fold cross-validation scheme was used, in which the total study group was randomly partitioned into six subgroups each one constituted by three infants with ADHD and two without ADHD. From these subgroups, the training sets $s D e$ (five subgroups) and the validation sets $s D v$ (one subgroup) were formed.

\section{RESULTS}

Cross-validation of the designed map structure established that correct classification rate for the system was $0,7 \pm 0,1095$. In particular, for an $s D e$ with 22 subjects $\left(14^{+}, 8^{-}\right)$and an $s D v$ with 6 subjects $\left(4^{+}, 2^{-}\right)$, seven clusters over the $S O M_{f}$ were found, defining three distinct regions of probability of positive ADHD in relation to the activated nodes. For example, the yellow cluster includes three activated nodes, two activated for positive ADHD, 
raising a confidence probability of 0,66 .

Regions of maximum probability were associated to clusters with $p=1$, moderate probability to those with $p=\frac{2}{3}$ and minimum to those with $p \leq \frac{1}{2}$. Blue and red circles marked over $S O M_{f}$ in Fig. 5 correspond to nodes activated by $s D v$ (with the subject ID within each circle total six) and marked as $\mathrm{ADHD}^{+}$or $\mathrm{ADHD}^{-}$.

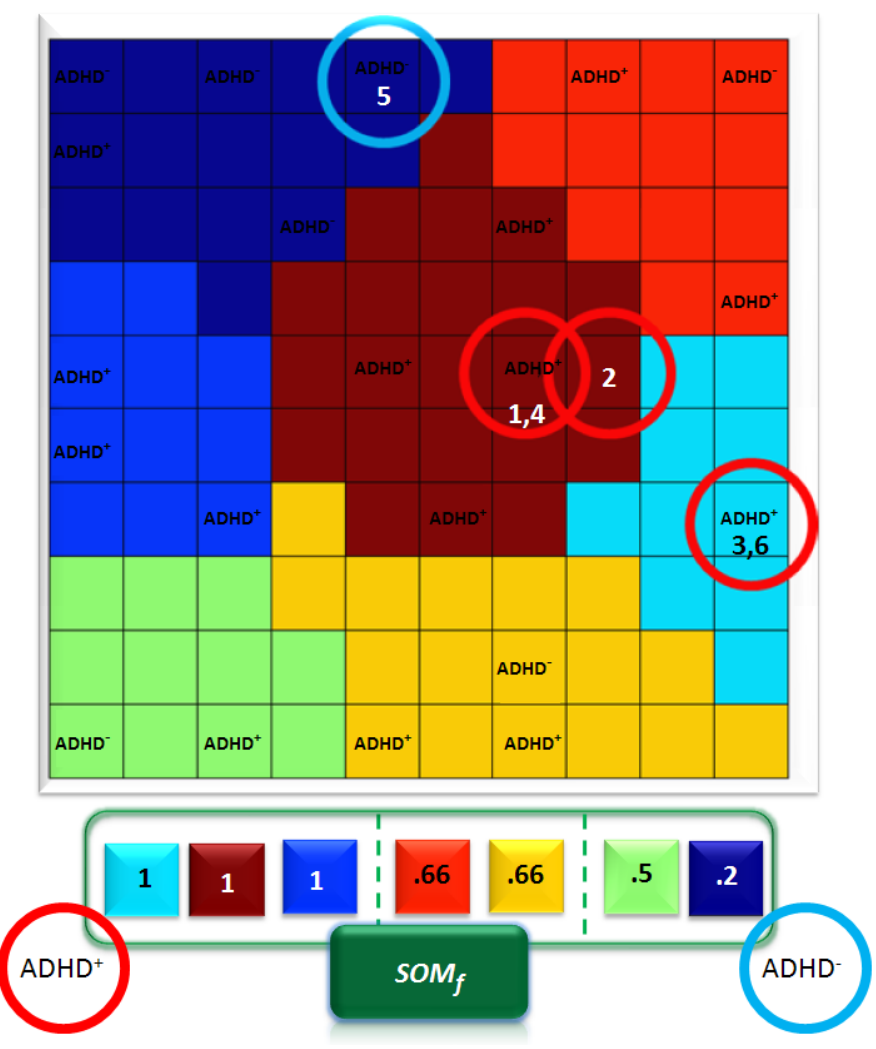

Fig. 5. $S O M_{f}$ grid, with color-coded clusters. Cluster confidence for positive ADHD representation is indicated in the row of boxes at the bottom of the map; dashed lines separate cluster groups of high-, medium- and low confidence. Circled nodes correspond to outputs of an unseen data set.

\section{DISCUSSION AND CONCLUSIONS}

Few contributions have proposed quantitative methods for the assessment of ADHD, beyond the studies on EEG power behavior in children with and without this disorder [1-6], so that advances on clinical grounds remain on the subjective, qualitative side. Information obtained from the porposed $S O M_{f}$ is independent to a large extent of subject collaboration or third-party intervention, which eliminates a major part of the bias inmersed in current diagnostic procedures.

The designed tool provides an attractive option to contribute to the body of diagnostic schemes related to the attentional disorder studied. Since for any computeraided diagnostic tool classification efficiency is dependent on the training set cardinality, it could be expected that accuracy would be increased as further evidence is collected. It appears that the choice of average power features adequately models the relevant cerebral activity for evaluating the presence of the ADHD, and therefore the SOM ensemble provides confident quantitative support to the clinical evaluator.

\section{ACKNOWLEDGMENTS}

Scholarship support for the corresponding author was provided by the National Council for Science and Technology (CONACyT), Mexico. Results from standardized tests and EEG recordings used in the present study were facilitated by psychologists Beatriz Rodriguez and Miguel Angel Rodriguez from the National Rehabilitation Institute, Mexico.

\section{REFERENCES}

[1] Brown C.R. Event-related potentials in attention-deficit/hyperactivity disorder of the predominantly inattentive type: An investigation of EEG-defined subtypes. International Journal of Psychophysiology 2005. 58(2005) : 94-6

[2] Carrillo H. M., González A. A., Gómez F. V. y Enríquez D. Z. Análisis cuantitativo del electroencefalograma para confirmar trastorno funcional frontal en niños con trastorno por déficit de atención con hiperactividad. Gac. Méd. Méx. 2007. 143(2007) : 391-400.

[3] Garcel J. R. Aportes del Electroencefalograma convencional y el análisis de frecuencias para el estudio del trastorno por déficit de atención. Salud mental 2003. 27(2004) : 22-7

[4] Clarke A.R. Quantitative EEG in low-IQ children with attentiondeficit/hyperactivity disorder. International Journal of Psychophysiology (2006). 117(2006) :1708-1711

[5] American Psychiatric Association. DSM-IV. Diagnostic and statistical manual of mental disorders. 4th ed. Washington, DC: American Psychiatric Association; 1994.

[6] Clarke A.R., et al. EEG in adults with Attention-Deficit/Hyperactivity Disorder. International Journal of Psychophysiology (2008) : 1-4

[7] T. Kohonen, K. Makisara, O. Simula and J. Kangas. Self-Organizing Maps: Optimization Approaches. Elsevier Science 1991. pp 981-990

[8] Proakis J.G. Manolakis D.G. Tratamiento digital de señales. Tercera edición. Ed. Prentice Hall. 2006. Spain. pp 632-640, 920-924

[9] Kohonen T. Self-Organizing Maps. Springer series in information sciences. 1995. Germany. pp 77-127, 143-170 y 191-204.

[10] Bishop C.M. Pattern Recognition. Springer series in Information Sciences. 2006. New York. pp 424-28. 


\section{Bibliografía}

La ciencia más útil es aquella; cuyo fruto es el más comunicable.

LEONARDO DA VINCI

[1] Brown C.R. Event-related potentials in attention-deficit/hyperactivity disorder of the predominantly inattentive type: An investigation of EEG-defined subtypes. International Journal of Psychophysiology 2005. 58(2005) : 94-6

[2] Morris, R. J., y Collier, S. J.,Attention deficit disorder and hyperactivity. Handbook of assessment in child psychopathology, New York, Plenum Press, 1987: 271-321

[3] Denckla, M. B., y Heilman, K. M., The syndrome of hyperactivity, clinical neuropsycology, New York, Oxford University Press, 1979. 574-597

[4] Clarke A.R. Quantitative EEG in low-IQ children with attentiondeficit/hyperactivity disorder. International Journal of Psychophysiology (2006). 117(2006) : 1708-1711

[5] Clarke A.R., et al. EEG in adults with Attention-Deficit/Hyperactivity Disorder. International Journal of Psychophysiology (2008) : 1-4

[6] Garcel J. R. Aportes del Electroencefalograma convencional y el análisis de frecuencias para el estudio del trastorno por déficit de atención. Salud mental 2003. $27(2004): 22-7$

[7] Carrillo H. M., González A. A., Gómez F. V. y Enríquez D. Z. Análisis cuantitativo del electroencefalograma para confirmar trastorno funcional frontal en niños con trastorno por déficit de atención con hiperactividad. Gac. Méd. Méx. 2007. 143(2007) : 391-400.

[8] Szatmari, P., Offord, D. R., y Boyle, M. H., Ontario child health study: Prevalence of attention deficit disorder with hyperactivity. Journal of Child Psychology and Psychiatry, 1989. (30) : 219-230.

[9] Luria, A. R. El cerebro en acción. Barcelona, Fontanella, 1979.

[10] Shue, K. L., y Douglas, V. I., Attention deficit hyperactivity disorder and the frontal lobe syndrome. Brain and Cognition, 20, 104-124. 
[11] Fournier, C., Análisis neuropsicológico de la epilepsia rolándica benigna y su comparación con el trastorno de hiperactividad en la edad escolar. Tesis doctoral. Madrid, Universidad Complutense, 1993.

[12] Manga, D., y Ramos, F., Neuropsicología de la edad escolar. Aplicaciones de la teoría de A. R. Luria a niños a través de la batería Luria-DNI, Madrid, Visor, 1991.

[13] Proakis J.G. Manolakis D.G. 8) Diseño de filtros digitales y 12) Estimación espectral de potencia en Tratamiento digital de señales. Tercera edición. Ed. Prentice Hall. 2006. España. pp 632-640 y 920-924

[14] Pérez López C. Técnicas de análisis multivariante de datos. Primera edición, Ed. Prentice Hall. 2006. España. pp 435-456

[15] Bishop C.M. Pattern Recognition. Springer series in Information Sciences. 2006. New York. pp 424-28.

[16] Kohonen T. 3) The Basic SOM, 5) Variants of SOM y 7) Applications en SelfOrganizing Maps. Springer series in information sciences. 1995. Germany. pp 77-127, 143-170 y 191-204.

[17] T. Kohonen, K. Makisara, O. Simula and J. Kangas. Self-Organizing Maps: Optimization Approaches. Elsevier science 1991. pp 981-990

[18] American Psychiatric Association. DSM-IV . Diagnostic and statistical manual of mental disorders. 4th ed. Washington, DC: American Psychiatric Association; 1994.

[19] Burrus, Gopinath, Guo. Introduction to Wavelets and Wavelet transforms A Primer. Prentice Hall. 1998. New Jersey.

[20] A. Belloch, B. Sandín y F. Ramos, 20) Psicopatología del desarrollo en Manual de Psicopatología. Ed. Mc-Graw Hill. 2004. España. pp 651-769.

[21] Lou HC, Andresen J, Steinberg B, McLaughlin T, Friberg L. The striatum in a putative cerebral network activated by verbal awareness in normals and ADHD children. Eur J Neurol. 1998 Jan;5(1):67-74.

[22] Zametkin AJ, Nordahl TE, Gross M. Cerebral glucose metabolism in adults with hyperactivity of childhood onset. 1990. N. Engl. J. Med. 323(20):1361-6.

[23] Matochik JA, Liebenauer LL, King AC, Szymansky HV, Cohen RM, Zametkin AJ. Cerebral glucose metabolism in adults with attention deficit hyperactivity disorder after chronic stimulant treatment. 1994. Am J Psychiatry 151(5):658-64.

[24] Piotr Olejniczak, Neurophysiologic Basis of EEG, J Clin Neurophysiol 2006; 23: 186-189 


\section{Soporte al diagnóstico de TDAH por búsqueda}

automatizada de patrones en el EEG

Tesis que para obtener el grado de Maestro en Ciencias (Ingeniería Biomédica)

Presenta

Ing. Biom. Federico Alba-Sánchez.

\section{Junio de 2011}

\section{Asesor: M. en I. Oscar Yañez Suárez}

Sinodales:

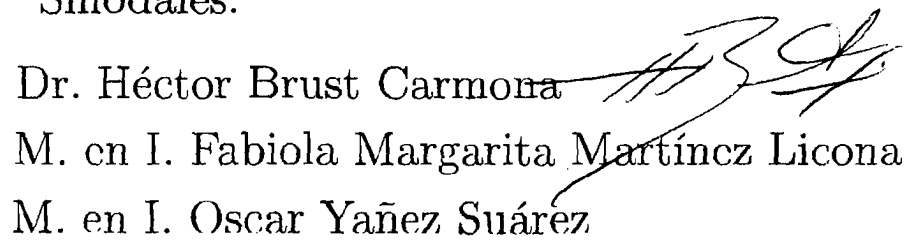
Instituto Nacional de Rahabilitación

Depto. de Ingenicría Eléctrica, UAM-I Depto. de Ingeniería Eléctrica, UAM-I
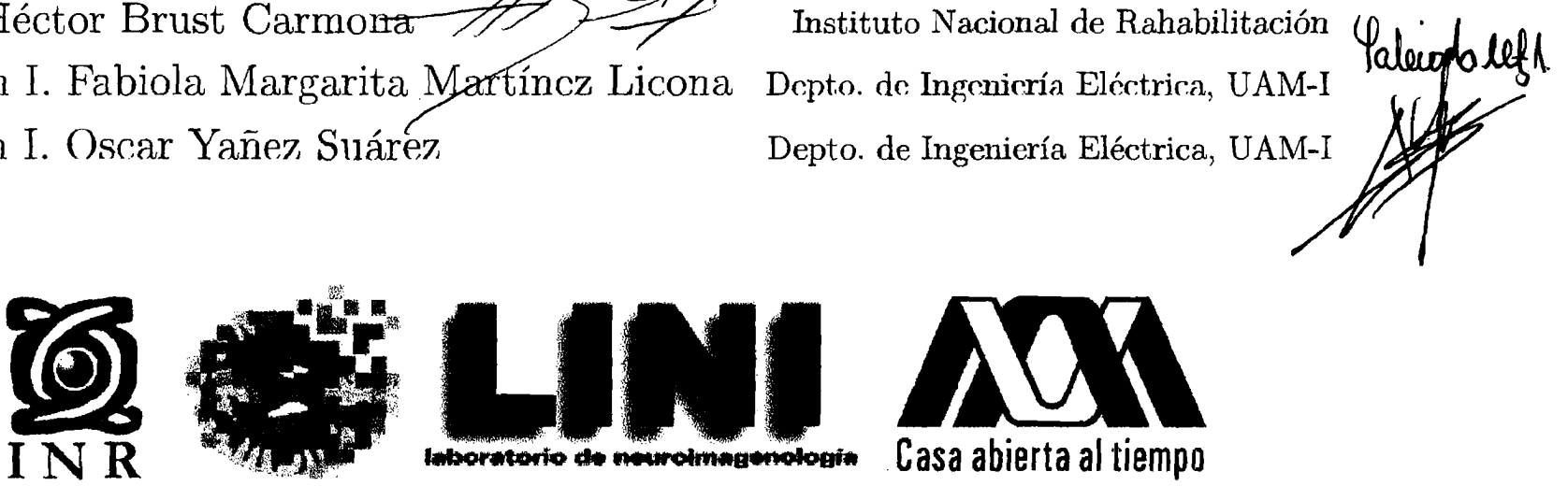

Casa abierta al tiempo

Universidad Autónoma Metropolitana

Unidad Iztapalapa

Ciudad de México 